\title{
Exoplanet Biosignatures: Observational Prospects
}

\author{
Yuka Fujii, ${ }^{1,2}$ Daniel Angerhausen, ${ }^{3,4}$ Russell Deitrick, ${ }^{5,6}$ Shawn Domagal-Goldman,,7 \\ John Lee Grenfell, 'Yasunori Hori, ${ }^{8}$ Stephen R. Kane, ${ }^{10}$ Enric Pallé, ${ }^{11,12}$ Heike Rauer, ${ }^{8,13}$ \\ Nicholas Siegler, ${ }^{14,15}$ Karl Stapelfeldt, ${ }^{14,15}$ and Kevin B. Stevenson ${ }^{16}$
}

\section{Abstract}

Exoplanet hunting efforts have revealed the prevalence of exotic worlds with diverse properties, including Earth-sized bodies, which has fueled our endeavor to search for life beyond the Solar System. Accumulating experiences in astrophysical, chemical, and climatological characterization of uninhabitable planets are paving the way to characterization of potentially habitable planets. In this paper, we review our possibilities and limitations in characterizing temperate terrestrial planets with future observational capabilities through the 2030s and beyond, as a basis of a broad range of discussions on how to advance "astrobiology" with exoplanets. We discuss the observability of not only the proposed biosignature candidates themselves but also of more general planetary properties that provide circumstantial evidence, since the evaluation of any biosignature candidate relies on its context. Characterization of temperate Earth-sized planets in the coming years will focus on those around nearby late-type stars. The James Webb Space Telescope (JWST) and later 30-meter-class ground-based telescopes will empower their chemical investigations. Spectroscopic studies of potentially habitable planets around solar-type stars will likely require a designated spacecraft mission for direct imaging, leveraging technologies that are already being developed and tested as part of the Wide Field InfraRed Survey Telescope (WFIRST) mission. Successful initial characterization of a few nearby targets will be an important touchstone toward a more detailed scrutiny and a larger survey that are envisioned beyond 2030. The broad outlook this paper presents may help develop new observational techniques to detect relevant features as well as frameworks to diagnose planets based on the observables. Key Words: Exoplanets-Biosignatures-Characterization-Planetary atmospheres-Planetary surfaces. Astrobiology $18,739-778$.

\footnotetext{
${ }^{1}$ NASA Goddard Institute for Space Studies, New York, New York, USA.

${ }^{2}$ Earth-Life Science Institute, Tokyo Institute of Technology, Ookayama, Meguro, Tokyo, Japan.

${ }^{3} \mathrm{CSH}$ Fellow for Exoplanetary Astronomy, Center for Space and Habitability (CSH), Universität Bern, Bern, Switzerland.

${ }_{5}^{4}$ Blue Marble Space Institute of Science, Seattle, Washington, USA.

${ }^{5}$ Department of Astronomy, University of Washington, Seattle, Washington, USA.

${ }^{6}$ NASA Astrobiology Institute's Virtual Planetary Laboratory.

${ }^{7}$ NASA Goddard Space Flight Center, Greenbelt, Maryland, USA.

${ }^{8}$ Department of Extrasolar Planets and Atmospheres (EPA), Institute of Planetary Research, German Aerospace Centre (DLR), Berlin, Germany.

${ }^{9}$ Astrobiology Center, National Institutes of Natural Sciences (NINS), Mitaka, Tokyo, Japan.

${ }^{10}$ Department of Earth Sciences, University of California, Riverside, California, USA.

${ }^{11}$ Instituto de Astrofísica de Canarias, La Laguna, Tenerife, Spain.

${ }_{12}^{12}$ Departamento de Astrofísica, Universidad de La Laguna, Tenerife, Spain.

${ }^{13}$ Center for Astronomy and Astrophysics, Berlin Institute of Technology, Berlin, Germany.

${ }^{14}$ Jet Propulsion Laboratory, California Institute of Technology, Pasadena, California, USA.

${ }_{16}^{15}$ NASA Exoplanet Exploration Office.

${ }^{16}$ Space Telescope Science Institute, Baltimore, Maryland, USA.
}

(C) Yuka Fujii et al., 2018; Published by Mary Ann Liebert, Inc. This Open Access article is distributed under the terms of the Creative Commons Attribution Noncommercial License (http://creativecommons.org/licenses/by-nc/4.0/) which permits any noncommercial use, distribution, and reproduction in any medium, provided the original author(s) and the source are credited. 


\section{Table of Contents}

1. Introduction 741

2. From Astrophysical Characterization to Astrobiological Characterization 743

2.1. The era of astrophysical characterization of exoplanets 743

2.2. The era of chemical characterization of exoplanets 743

2.3. The era of astrobiological characterization of exoplanets 744

3. Characterizing Transiting Planets 746

3.1. Astrophysical characterization 747

$\begin{array}{lll}\text { 3.1.1. } & \text { Method and sensitivity } & 747\end{array}$

3.1.2. Opportunities through $2030 \quad 747$

3.2. Chemical/Climatological characterization: Transmission spectroscopy 749

3.2.1. Method and sensitivity $\quad 749$

3.2.2. What can be studied?

3.2.3. Opportunities through $2030 \quad 751$

3.3. Chemical/Climatological characterization: Eclipse spectroscopy 752

3.3.1. Method and sensitivity $\quad 752$

3.3.2. What can be studied?

3.3.3. Opportunities through $2030 \quad 753$

4. Characterizing Planets with General Orbital Inclination 753

4.1. Astrophysical characterization 753

4.1.1. Methods and sensitivity

4.2. Chemical/Climatological characterization: Phase curves 754

4.2.1. Method and sensitivity $\quad 754$

4.2.2. What can be studied?

4.2.3. Opportunities through $2030 \quad 754$

4.3. Chemical/Climatological characterization: High-contrast imaging 754

4.3.1. Method and sensitivity $\quad 754$

$\begin{array}{ll}\text { 4.3.2. What can be studied? } & 757\end{array}$

4.3.3. Opportunities through $2030 \quad 759$

4.4. Chemical/Climatological characterization: Spectral separation 760

4.4.1. Method and sensitivity $\quad 760$

4.4.2. What can be studied?

4.4.3. Opportunities through $2030 \quad 760$

5. Contextual Information 760

5.1. Properties of the host star 761

5.1.1. Mass, radius, SED in the visible/IR range 761

5.1.2. Activity (SED in UV, X-ray, superflares) 761

5.2. Orbital architecture of the planetary system 761

5.3. Characterization of larger planets in the system 762

6. Prospects Beyond $2030 \quad 762$

6.1. Mission concepts currently being studied in the United States 762

6.1.1. Habitable Exoplanet Imaging Mission (HabEx) 762

6.1.2. Large UltraViolet Optical and InfraRed surveyor (LUVOIR) 762

6.1.3. Origins Space Telescope (OST)

6.2. Ideas for the far future 763

6.2.1. Direct imaging in the mid-IR $\quad 763$

$\begin{array}{ll}\text { 6.2.2. } & \text { ExoEarth Mapper }\end{array}$

6.2.3. Telescope on the Moon $\quad 764$

6.2.4. One-hundred-meter-class ground-based telescope 764

7. Summary: Ideal Timeline 764

Acknowledgments

Author Disclosure Statement

References 


\section{Introduction}

I N THE ENDEAVOR to discover life beyond the Solar System, the most critical step is to detect photometric, spectroscopic, and/or polarimetric properties of "potentially habitable exoplanets" and search for features related to life. The ways in which such observations can be utilized to detect life at various confidence levels are described in the other manuscripts in this issue (Catling et al., 2018; Meadows et al., 2018; Schwieterman et al., 2018; Walker et al., 2018). The idea of building a space-based direct-imaging observatory specifically aimed at detecting signs of life on Earth-like planets dates back to the 1990s (e.g., Burke, 1992; Elachi et al., 1996), which elicited the Terrestrial Planet Finder (TPF) mission studies by NASA (Beichman et al., 1999; Lawson et al., 2007; Levine et al., 2009) and Darwin mission concepts of ESA (Léger et al., 1996; Fridlund, 2000). The Advanced Technology Large-Aperture Space Telescope (ATLAST) concept represents a general-purpose observatory capable of exoplanet direct-imaging with even larger apertures (Postman et al., 2009) and was later updated as the High Definition Space Telescope (HDST, AURA, http://www .hdstvision.org). While the last Astrophysics Decadal Survey of the United States did not prioritize any of these concepts (https://www.nap.edu/catalog/12951/new-worlds-new-horizonsin-astronomy-and-astrophysics), it did recommend exoplanet technology development as its top medium-class investment.

Since these early mission studies, a huge expansion of exoplanet science has taken place thanks to discoveries and initial characterization made by radial velocity, transit, microlensing surveys, transit spectroscopy of close-in planets, and direct imaging of uninhabitable self-luminous exoplanets. These observations have revealed thousands of exoplanets, allowing for analyses of demographic trends in the exoplanet population. Of particular interest from an astrobiological viewpoint is the occurrence rate of terrestrial planets in socalled habitable zones (HZs), that is, the circumstellar region in which liquid water could exist on the surface of a terrestrial planet (Kasting et al., 1993). This rate is conventionally represented by $\eta_{\oplus}$, and estimates were obtained employing various criteria for the "terrestrial" size and for the range of HZs (Catanzarite and Shao, 2011; Traub, 2012; Bonfils et al., 2013; Dressing and Charbonneau, 2013, 2015; Gaidos, 2013; Kopparapu, 2013; Petigura et al., 2013; Morton and Swift, 2014; Burke et al., 2015; Silburt et al., 2015; Zsom, 2015). While the estimates span a range from a few percent up to the order of unity reflecting the differences in the data sets and the thresholds for the targets, it is now established that Earthsized planets in HZs are not rare. Meanwhile, the analyses of the mass-radius relationship of close-in planets (period shorter than $\sim 100$ days), have revealed that most of the planets with radii below $1.5-2 R_{\oplus}\left(R_{\oplus}\right.$ is the Earth radius) are consistent with rocky/metallic composition, while bigger planets have large scatter in bulk density with a substantial fraction of volatile-rich planets (e.g., Weiss and Marcy, 2014; Rogers, 2015; Kaltenegger, 2017). Interestingly, a recent analysis indicates a gap in population between the planets smaller than $\sim 1.5 R_{\oplus}$ and those larger than $\sim 2 R_{\oplus}$ (Fulton et al., 2017). A few probably terrestrial planets around HZs have already been discovered in the solar neighborhood: Proxima Centauri b, an Earth-mass planet receiving 65\% of the incident flux received by the Earth, only $1.3 \mathrm{pc}$ away
(Anglada-Escudé et al., 2016); GJ 273 b, a planet a few times as massive as Earth with an incident flux similar to that received by Earth, $3.8 \mathrm{pc}$ away (Astudillo-Defru et al., 2017b); seven transiting Earth-sized planets around an ultra-cool star TRAPPIST-1, three to four of which could conceivably be habitable, 12 pc away (Gillon et al., 2017); and LHS 1140 b, a large terrestrial planet 12 pc away (Dittmann et al., 2017).

In parallel, substantial technological and methodological progress is being made through the characterization of larger and/or hotter exoplanets. Recently proven observational techniques to characterize planetary atmospheres include the usage of temporal variation to map the heterogeneity of planetary photospheric surfaces (e.g., Knutson et al., 2007, 2012; de Wit et al., 2012; Majeau et al., 2012; Demory et al., 2013, 2016) and the usage of the crosscorrelation analysis on high-resolution spectra to extract Doppler-shifted lines due to the planetary atmosphere (e.g., Snellen et al., 2010; Birkby et al., 2013; Konopacky et al., 2013). Lessons on data reduction processes and atmospheric retrieval techniques are being learned (see Deming and Seager, 2017, for a review). Numerical simulations are also used to further develop the data analysis techniques of spectroscopic data (e.g., Line et al., 2013; Line and Parmentier, 2016; Rocchetto et al., 2016; Deming and Sheppard, 2017) and photometric light curves (see Cowan and Fujii, 2017, for a review). The starlight suppression technologies for high-contrast imaging have been advanced by the successful ground-based direct-imaging observations using adaptive optics and coronagraphs (e.g., Kalas et al., 2008; Marois et al., 2008; Lagrange et al., 2010; Kuzuhara et al., 2013; Macintosh et al., 2015). Starshades have emerged as a viable alternative approach to coronagraphs (Cash, 2006).

As these observations have progressed, theoretical work has been exploring the properties and diversity of temperate terrestrial planets, which could eventually be studied through similar techniques. The list of potential biosignatures continues to grow and includes the spectral features of atmospheric (volatile) molecules originated from possible life (e.g., $\mathrm{O}_{2}, \mathrm{O}_{3}, \mathrm{CH}_{4}, \mathrm{~N}_{2} \mathrm{O}, \mathrm{CH}_{3} \mathrm{Cl}$ ), the reflectance spectra of biological surfaces (e.g., vegetation's red edge, reflectance spectra of pigments), and the temporal variation of these signatures (see a review by Schwieterman et al., 2018, and references therein). It has also been recognized that the proposed potential biosignatures contain the risk of false positives (i.e., they can be produced nonbiologically under particular situations). Therefore, identifying an inhabited planet with confidence also requires as much contextual information as possible to evaluate the prospect of a non-biological origin of detected biosignature candidates and to find auxiliary evidence consistent with a biological origin (see Meadows et al., 2018, for a discussion on how $\mathrm{O}_{2}$ would work as a biosignature, and see Catling et al., 2018, for a framework to assess potential biosignatures). The successful interpretation will require significant advances in our ability to model both inhabited and uninhabited worlds (see discussions in Walker et al., 2018) as well as the detailed observational data.

Founded on these ongoing observational, technological, and theoretical developments, new space-based missions and ground-based facilities will come into play in the near future. The planned new telescopes most relevant to the investigations of potentially habitable planets are listed in 
Table 1, together with their specifics and the expected usage. In this paper, we overview the capabilities of these future missions as well as the observational methods they will employ, and discuss what kind of properties of potentially habitable exoplanets could be observationally constrained. We do not intend to prioritize future projects or observational techniques. Instead, our aim is to share the ongoing efforts and limitations in exoplanet observations with a broad range of readers involved in astrobiology, so that we can be on the same page to think collaboratively about how to make the most of future opportunities to deduce useful information about planets.

In this paper, we use the term "potentially habitable exoplanets" to imply two properties of such planets: (1) terrestrial, i.e., inferred to have a well-defined surface and no voluminous gaseous envelope, and (2) in the $\mathrm{HZ}$ of their stars. For condition (1), we focus on small planets roughly up to $\sim 2 R_{\oplus}$ in radius and up to $\sim 10 M_{\oplus}$ in mass where $M_{\oplus}$ is the Earth mass, referring to the recent observational evidence of the radius/mass range of planets consistent with no voluminous gaseous envelope (Kaltengger, 2017). As for (2), to the first order the orbital distance of HZs scales with the square root of the stellar luminosity, and rough estimates tell that they are around 1 AU for solar twins and around 0.01-0.3 AU for M-type stars $\left(M_{\star} \lesssim 0.5 M_{\odot}\right.$, $L_{\star} \sim 10^{-4} \sim 10^{-1} L_{\odot}$ where $M_{\star}$ and $L_{\star}$ are stellar mass and luminosity, respectively, and $M_{\odot}$ and $L_{\odot}$ denote the solar values). Note that the exact location of the $\mathrm{HZ}$ of a given star depends on many factors including the stellar spectrum, planetary rotation, atmospheric properties, the initial amount of water, and the evolutionary history of the surface environment (e.g., Kasting et al., 1993; Abe et al., 2011; Pierrehumbert and Gaidos, 2011; Kopparapu et al., 2013, 2014, 2016; Leconte et al., 2013; Wolf and Toon, 2014, 2015; Yang et al., 2013; Zsom et al., 2013; Kadoya and Tajika, 2014; Ramirez and Kaltenegger, 2014, 2016, 2017; Wolf, 2017). Outside these HZs, planets may not necessarily be inhospitable, yet their habitable environments are more likely to be confined to the subsurface ( $c f$. the "internal" oceans of Europa and Enceladus) and thus are probably more difficult to observe across interstellar distances.

The organization of this chapter is as follows. In Section 2 , we broadly describe the overall trend in exoplanet observations, which we expect to evolve from a focus on the astrophysical characterization of exoplanets toward their chemical, climatological, and astrobiological characterization. Then we move on to how individual potentially habitable planets will be characterized from various aspects. We discuss transiting planets (Section 3) and planets with general orbital inclination (Section 4) separately, as the former enable some unique methods for astrophysical and chemical characterization and will be the prime targets in the coming few years. In each of these two sections, we review the methods for astronomical (mass, radius, orbit) and chemical/climatological (atmosphere, surface, etc.) characterizations, and the planned observational projects that may make use of the methods. While we try our best to reflect the state of the field at the time of writing, the specifics of the future missions are subject to change. Section 5 is devoted to how the contextual information, including host star properties and planetary system properties, will be obtained and how it will help in evaluating the planetary conditions. In Section 6, we introduce the mission concepts under development that envision commencing of operation beyond 2030, and explore more ambitious possibilities presented in the literature that could be planned further in the future. Lastly, Section 7 concludes this paper by placing the projects in a timeline and discussing the work to be done.

Table 1. Planned New Observatories Most Relevant

to Characterization of Potentially Habitable Planets

\begin{tabular}{|c|c|c|c|c|c|c|}
\hline & $\begin{array}{l}\text { Expected } \\
\text { start }\end{array}$ & $\begin{array}{l}\text { Spacel } \\
\text { Ground }\end{array}$ & Aperture & $\begin{array}{l}\text { Purpose/Usage for potentially } \\
\text { habitable planets }\end{array}$ & Instruments & Wavelength \\
\hline TESS & $2018^{\mathrm{a}}$ & space & $\mathrm{b}$ & $\begin{array}{l}\text { Discover transiting planets } \\
\text { orbiting bright stars }\end{array}$ & photometry & $0.6-1.0 \mu \mathrm{m}$ \\
\hline CHEOPS & 2018 & space & $32 \mathrm{~cm}$ & $\begin{array}{l}\text { Provide precise radii of known } \\
\text { exoplanets, find transits } \\
\text { of RV planets }\end{array}$ & photometry & $0.4-1.1 \mu \mathrm{m}$ \\
\hline JWST & 2020 & space & $6.5 \mathrm{~m}$ & $\begin{array}{l}\text { Transmission/eclipse } \\
\text { spectroscopy, Phase curves }\end{array}$ & $\begin{array}{l}\text { spectroscopy } \\
\text { (NIRISS, NIRSpec, } \\
\text { NIRCam, MIRI) }\end{array}$ & $0.6-28.5 \mu \mathrm{m}$ \\
\hline GMT & 2023 & ground & $24.5 \mathrm{~m}$ & Transmission spectroscopy, & spectroscopy, & $0.3 \mu \mathrm{m}-$ \\
\hline ELT & 2024 & ground & $39.3 \mathrm{~m}$ & High-contrast imaging, & coronagraphy & \\
\hline TMT & 2027 & ground & $30 \mathrm{~m}$ & $\begin{array}{l}\text { High-contrast imaging with } \\
\text { high-resolution spectroscopy }\end{array}$ & & \\
\hline PLATO & 2026 & space & $\mathrm{c}$ & $\begin{array}{l}\text { Discover and characterize } \\
\text { transiting planets around bright } \\
\text { stars, including planets in HZs } \\
\text { of solar-type stars }\end{array}$ & photometry & $0.5-1.05 \mu \mathrm{m}$ \\
\hline WFIRST & 2025 & space & $2.4 \mathrm{~m}$ & $\begin{array}{l}\text { High-contrast imaging (+ Discover } \\
\text { planets by microlensing) }\end{array}$ & $\begin{array}{l}\text { coronagraphy, } \\
\text { low-resolution } \\
\text { spectroscopy }\end{array}$ & $0.6-0.95 \mu \mathrm{m}(\mathrm{CGI})$ \\
\hline
\end{tabular}

\footnotetext{
${ }^{a}$ Launched.

${ }^{\mathrm{b}} 4$ cameras with $10.5 \mathrm{~cm}$ aperture each.

${ }^{c} 26$ cameras with $12 \mathrm{~cm}$ aperture each.
} 


\section{From Astrophysical Characterization to Astrobiological Characterization}

In this section, we briefly summarize how the exoplanet community as a whole plans to advance toward astrobiological investigations of exoplanets with future missions. This includes a description of discoveries our community anticipates occurring in three broad eras of exoplanet observations: (1) astrophysical characterization, (2) chemical/ climatological characterization, and (3) astrobiological characterization. Astrobiological characterization can be seen as astrophysical, chemical, and climatological characterizations particularly for potentially habitable planets. Figure 1 is a schematic showing the relations among these regimes. The arrows of various missions reach in to the "astrobiological characterization" region to an extent reflecting approximately the similarity of their targets to Earth; thus, the missions primarily for potentially habitable planets around M-type stars are slightly shortened. This section can serve as a preview of the rest of the manuscript, in which we detail the methodologies and the specifics of the future projects to discuss how individual planets of astrobiological interest will be characterized.

\subsection{The era of astrophysical characterization of exoplanets}

We are in the golden age of the era focused on the detection and astrophysical characterization of exoplanets. After the pioneering Convection, Rotation and planetary Transits (CoRoT) mission demonstrated precision photometry from space, the larger aperture and higher photometric precision of the Kepler mission enabled thousands of planets to be discovered, including numerous Earth-sized planets. For many of these worlds, we have measured both size and mass, knowledge of which allows for inferences on the bulk composition of these planets. The large sample sizes have also allowed for trends to be uncovered in exoplanet populations. The combination of the exoplanet demographics and the simulations of bulk composition and density has led to the inference that, at least for close-in planets, there are three classes of planet size/mass: (1) planets with a rock-dominated composition that have small masses and radii; (2) planets with a gas-dominated composition that have large masses and radii; and (3) planets with intermediate sizes that have a composition which is dominated by neither rock nor gas.

The discoveries made during this era have also included multiple surprises in the orbital and size properties of planets. Hot Jupiters with large masses and short orbits were thought to be improbable if not impossible yet were the first planets discovered around main sequence stars (Mayor and Queloz, 1995). Circumbinary planets were proposed in science fiction lore yet were considered dubious by the astrophysics community until discovered by the Kepler mission (Doyle et al., 2011). The era of astrophysical characterization began with biases toward planets larger than Jupiter and orbits shorter than Mercury's. Over time, detection techniques have improved to allow detections of planets with potentially habitable conditions. This began with discoveries by the Kepler mission (Borucki et al., 2011), has continued with ground-based surveys (e.g., Anglada-Escudé et al., 2012, 2016; Astudillo-Defru et al., 2017b; Dittmann et al., 2017; Gillon et al., 2017), and will continue further with ground-based measurements as well as the upcoming Transiting Exoplanet Survey Satellite (TESS) and PLAnetary Transits and Oscillations of stars (PLATO) missions (Section 3.1.2). This "census" of astrophysical properties of exoplanets will be complemented well by the Gaia astrometric survey, which will be biased toward the detection of planets with orbits that extend beyond the HZ, and Wide Field InfraRed Survey Telescope (WFIRST) microlensing surveys, which will be sensitive to the intermediate orbital regions. The latter will provide greater completeness to our survey of the abundance of potentially habitable worlds.

\subsection{The era of chemical characterization of exoplanets}

Some of the recent exoplanet discoveries, and those anticipated from TESS and the CHaracterising ExOPlanet Satellite

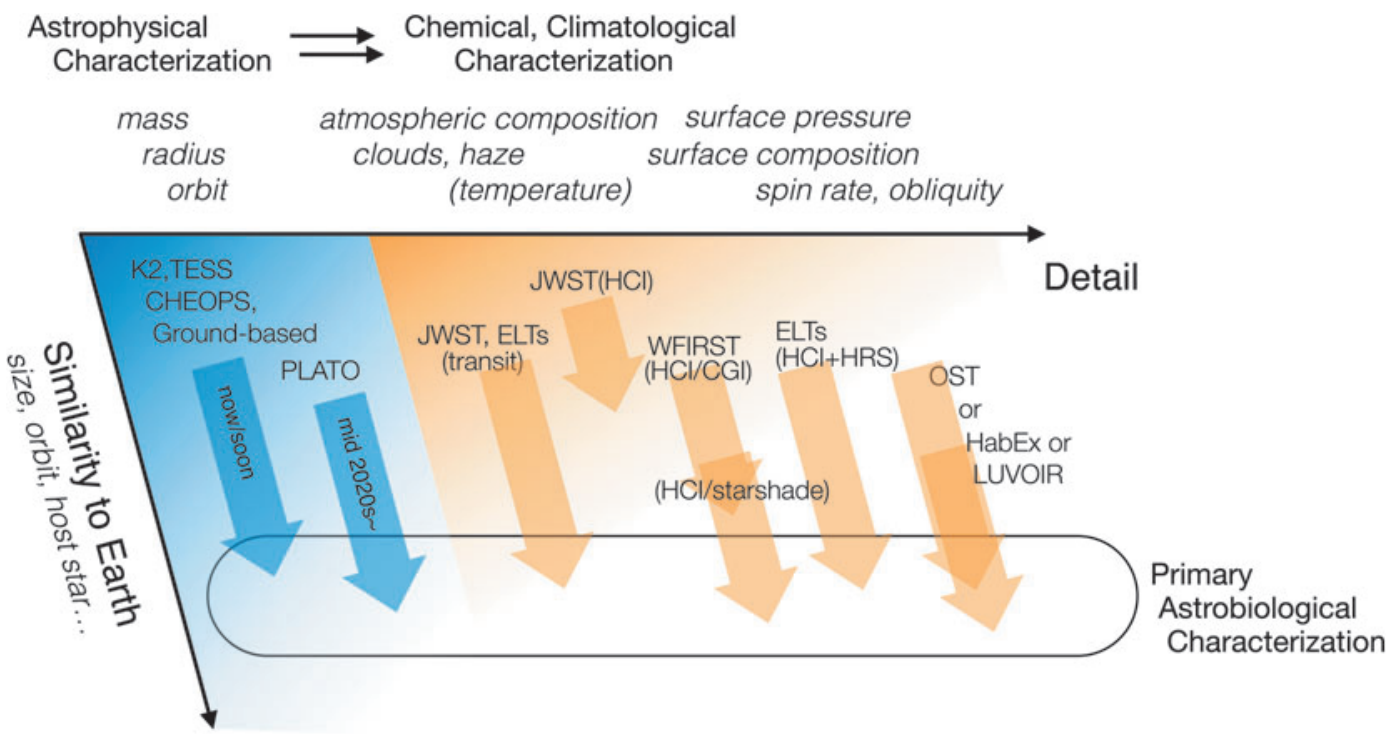

FIG. 1. Schematic figure showing astrophysical, chemical, climatological, and astrobiological characterizations and the possible contributions from the current and future missions ( $\mathrm{HCI}=$ high-contrast imaging; $\mathrm{HRS}=$ high-resolution spectroscopy). 
(CHEOPS), will present the community with a pivot point to the next era of exoplanet characterization, which will be focused on chemical composition. Discoveries of nearby transiting worlds will enable follow-up transmission spectroscopy observations (Section 3.2). In principle, the method is similar to the means by which transiting planets are discovered, but higher sensitivities allow transit events to be measured at multiple wavelengths. As the wavelength dependence of the transit is a function of the atmosphere's opacity and scattering properties, this method will identify the chemical composition of exoplanet atmospheres. Further information can be obtained from spectroscopy of planetary eclipses, which extracts planetary dayside emissions (Section 3.3), and/or based on the phase curves of exoplanets, which probe the heterogeneity of the atmosphere and enable us to map some features (Section 4.2). While these kinds of chemical analyses have been done with the Hubble Space Telescope (HST) (e.g., Charbonneau et al., 2002; Vidal-Madjar et al., 2003; Kreidberg et al., 2014), Spitzer Space Telescope (e.g., Grillmair et al., 2007; Richardson et al., 2007; Knutson et al., 2007), SOFIA (e.g., Angerhausen et al., 2015), and ground-based observatories (e.g., Redfield et al., 2008), they will accelerate when the James Webb Space Telescope (JWST) launches (Section 3.2.3).

These observations by JWST should constitute the start of the golden era for the chemical characterization of exoplanets, which will continue with multiple observatories and techniques. JWST should have enough observation time for direct imaging of dozens of young gas giants and should be able to measure their chemical inventories. The next generation of ground-based instruments (Section 3.1.2) will also enable detailed transmission spectroscopy of transiting gaseous planets and direct-imaging observations of young gaseous planets in distant orbits. Ultimately, the $30 \mathrm{~m}$ class groundbased telescopes (also referred to as "Extremely Large Telescopes" or ELTs for short; Sections 3.2.3, 4.3.3, and 4.4.3) will eventually carry out more sensitive transit spectroscopy and direct imaging of many exoplanets, potentially down to subNeptune-sized planets. Future space missions dedicated to spectroscopy of exoplanets, the Fast Infrared Exoplanet Spectroscopy Survey Explorer (FINESSE; Swain, 2012) and Atmospheric Remote-sensing Infrared Exoplanet Largesurvey (ARIEL; Tinetti et al., 2016) plan to conduct a chemical survey of 500 and 1000 transiting planets, respectively, in the 2020s. The wealth of data on the atmospheric composition from these observatories will provide crucial insights into the formation histories of planetary systems, putting our own solar system into a broader context.

\subsection{The era of astrobiological characterization of exoplanets}

Chemical characterization with JWST and the ELTs will also initiate the era of astrobiological characterization through a confirmation of habitable conditions and a search for signs of life on potentially habitable exoplanets. JWST should be capable of characterizing the atmospheric composition of at least one potentially Earth-like exoplanet (Stevenson et al., 2016), while the updated instruments with existing ground-based telescopes and future ELTs also plan to probe their atmospheres with transmission spectroscopy (Section 3.2), highcontrast imaging (Section 4.3), or high-contrast high-resolution observations (Section 4.4). The observations with these facilities will likely be limited to a few planets in orbit around Mtype stars. These stars are smaller than the Sun and have a relatively larger transit depth for an Earth-sized planet and a planet-to-star contrast ratio that is a few orders of magnitude better than the contrast ratio of Earth-like planets to Sun-like stars. The habitability of such worlds has been brought into question based on complications stemming from the star's high-energy radiation (Ramirez and Kaltenegger, 2014; Luger and Barnes, 2015; Airapetian et al., 2017) and from the climate effects of synchronously rotating planets (Joshi et al., 1997; Joshi, 2003; Wordsworth et al., 2011; Barnes et al., 2013). Regardless of the outcome, however, this will be the first time such observations are possible for temperate Earth-sized planets around other stars.

The golden age in the era of astrobiological characterization will likely require a space-based flagship mission that includes biosignature detection as a major design driver of the mission's architecture. Historically, the study of such missions has focused on direct-imaging missions such as TPF-C, TPF-I, the New Worlds Observer, THEIA, and Darwin. However, biosignatures could also be detected via transit transmission/ emission spectroscopy, if the observatory has sufficiently low noise characteristics. Currently, NASA is studying three flagship mission concepts in advance of the next US Decadal Survey, which all include a search for biosignatures in their design drivers: Habitable Exoplanet Imaging Mission (HabEx), Large UltraViolet Optical and InfraRed surveyor (LUVOIR), and Origins Space Telescope (OST) (Section 6.1). OST is a general mid-IR observatory and is being designed with transit spectroscopy of potentially habitable worlds in mind; it will be more sensitive than JWST and should extend observations to longer wavelengths and larger numbers of planets than what JWST can access. LUVOIR does not have transit spectroscopy as a central driver, but its large collecting area ( $>8 \mathrm{~m}$ mirror) should increase sensitivity and do so at a wavelength range complementary to (shorter than) JWST's. However, the primary targets of these transit spectroscopy observations would still be planets orbiting M-type stars. Both HabEx and LUVOIR aim to characterize terrestrial planets in the HZs around a variety of nearby stars, with most targets being F-, G-, or K-type stars, via direct-imaging spectroscopy, and to conduct a range of general astrophysics observations that would place the exoplanet spectra in the context of the host star, comparative planetology, and cosmological history; they differ in their levels of quantitative ambition. These direct imaging observations would probe deeper into exoplanet atmospheres, at some wavelengths down to the surface. Thus, the missions designed with this technique in mind would be able to assess exoplanetary properties that will be difficult or impossible to otherwise observe. These three missions are discussed in more detail in Section 6.1.

The way individual planets of astrobiological interest are characterized will not necessarily proceed monotonically from astrophysical to chemical and climatological characterization. The possibilities to measure specific planetary properties depend on many factors, including whether the planets are transiting or not. Thus, we discuss prospects for transiting planets and planets with general orbital inclination separately in the following two sections. These prospects for future observations also depend on the spectral type of the host star. Therefore, we consider solar-type (F-, G-, K-type) 


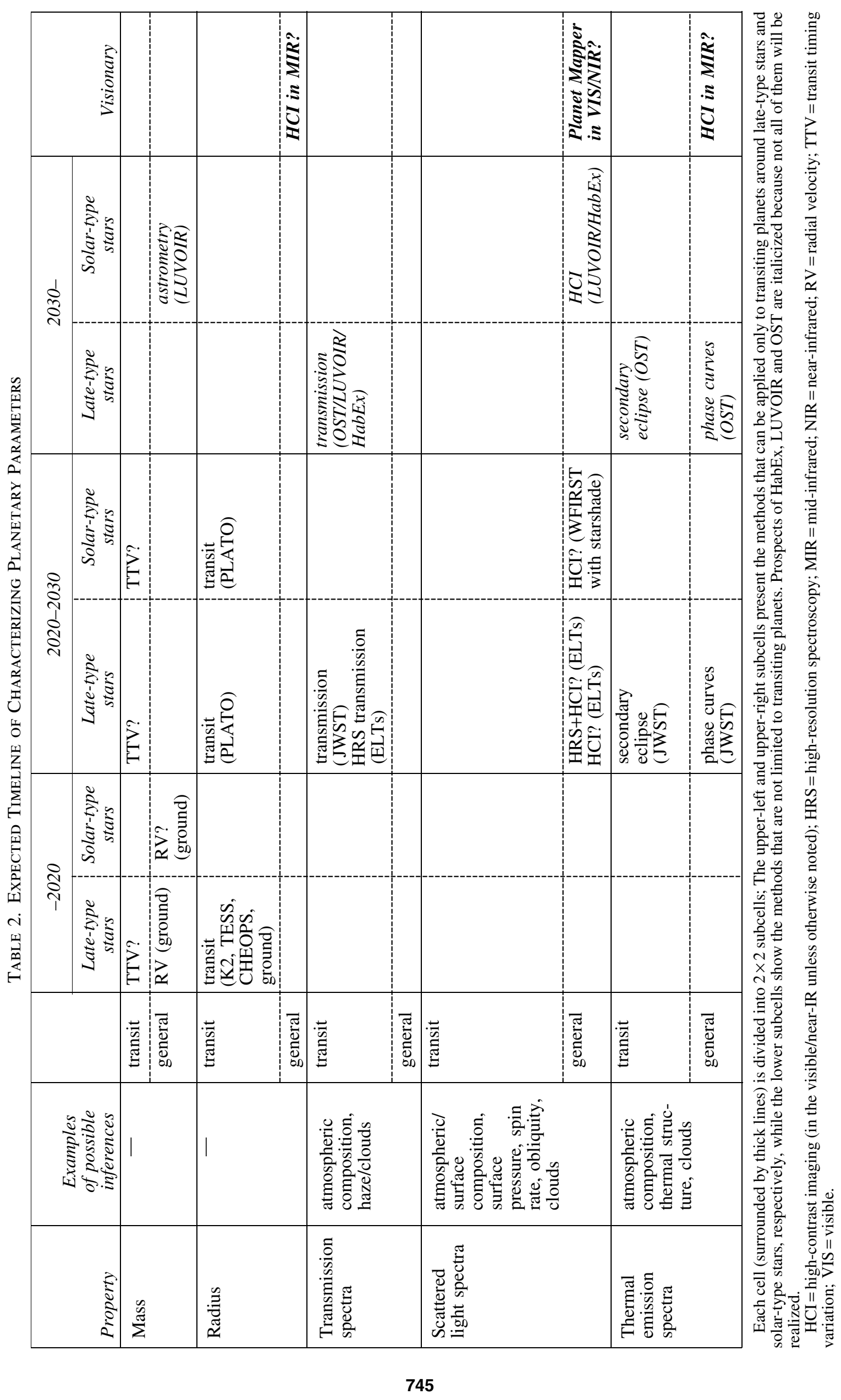



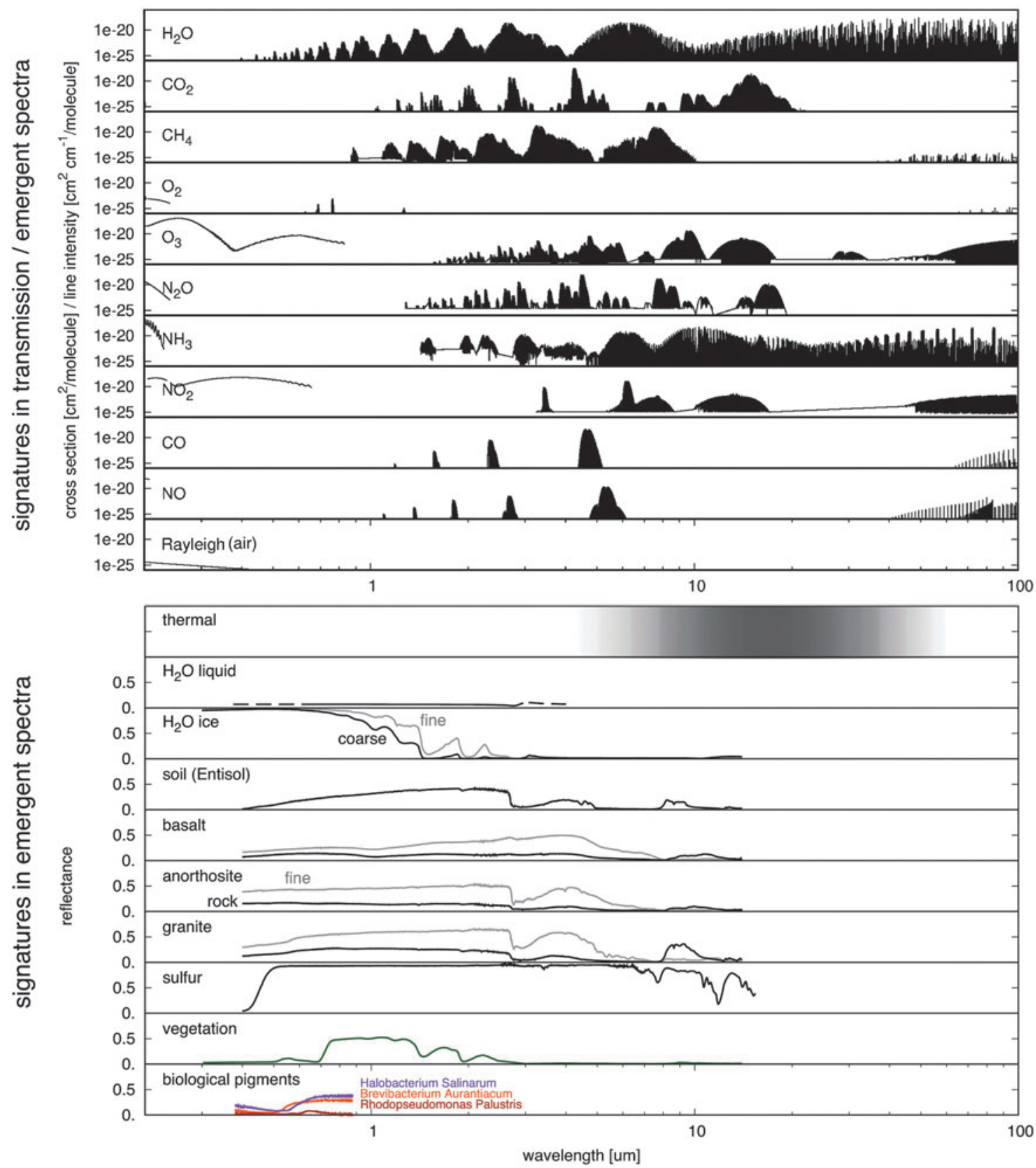

FIG. 2. Examples of atmospheric and surface spectral features of temperate terrestrial planets. Upper panel: Atmospheric signatures, which can in principle be probed through both transmission spectra and emergent spectra. The continuous features of molecules at shorter wavelengths are absorption cross section at approximately $300 \mathrm{~K}$, taken from MPI-Mainz UV/VIS Spectral Atlas of Gaseous Molecules of Atmospheric Interest (Keller-Rudek et al., 2013), shown in log scale from $10^{-26}$ to $10^{-16} \mathrm{~cm}^{2} / \mathrm{molecule}$. Original data sources are Yoshino et al. (1988) for $\mathrm{O}_{2}$; Brion et al. (1998) for $\mathrm{O}_{3}$; Selwyn et al. (1977) for $\mathrm{N}_{2} \mathrm{O}$; Cheng et al. (2006) for $\mathrm{NH}_{3}$; Mérienne et al. (1997), Coquart et al. (1995), and Vandaele et al. (1998) for $\mathrm{NO}_{2}$. The lines at longer wavelengths are line intensities at $296 \mathrm{~K}$ and 1 atm in $\log$ scale from $10^{-26}$ to $10^{-16} \mathrm{~cm}^{2} \mathrm{~cm}^{-1} /$ molecule taken from the HITRAN2012 database (Rothman et al., 2013). Lower panel: thermal radiation and reflectance spectra of surface materials, which can be probed in emergent light. The reflectance is shown in linear scale from 0 to 1 . All data but biological pigments are taken from the ECOSTRESS Spectral Library (Baldridge et al., 2009; Meerdink et al., unpublished data). The data of biological pigments are from VPL spectral databases (Schwieterman et al., 2015).

stars and late-type (M-type) stars as two representative classes to give a rough idea. We summarize the prospects for each class (transiting or nontransiting planets around solar-type or late-type stars) in Table 2. Note that in reality the spectral type of the star is continuous, and the scope of each observational method does not necessarily follow this classification strictly. Figure 2 summarizes some spectral signatures of interest, which will be discussed later in the paper.

\section{Characterizing Transiting Planets}

In this section, we focus on the methods to characterize potentially habitable planets that are applicable to transiting 
ones. Measurements of radius, mass, and the orbital elements are referred to as astrophysical characterization and discussed in Section 3.1. Chemical, climatological characterization through transmission spectroscopy and eclipse spectroscopy are discussed in Sections 3.2 and 3.3, respectively. In each of these subsections, we review the method, sensitivity consideration, and the planetary properties that, in principle, can (or cannot) be studied using the method, followed by the prospects with the future observational facilities to be available by 2030 .

\subsection{Astrophysical characterization}

3.1.1. Method and sensitivity. Radius. The radius of a transiting planet is measured primarily from the transit depth. (Strictly speaking, the transit depth tells us the planetary radius relative to the star, thus the stellar radius needs to be well constrained in order to obtain accurate information on the planet's radius.)

Mass. Masses of transiting planets have been measured through two methods: the radial velocity (RV) method and the transit timing variation (TTV) method. The RV method, which detects the reflex motion of the star due to the planetary orbital motion through the Doppler shift of the high-resolution stellar spectra, measures the product of the planetary mass and sine of the orbital inclination angle $(i)$, but because the inclination of transiting planets can be determined $\left(i \sim 90^{\circ}\right)$, the true mass is obtained. A difficulty with potentially habitable planets is that the variation of stellar RV they produce is generally much smaller than most of the successful RV observations to date. The amplitude of stellar $\mathrm{RV}$ variation due to a planet, $K$, is approximately

$$
K \sim 9 \mathrm{~cm} / \mathrm{s}\left(\frac{M_{\mathrm{p}} \sin i}{M_{\oplus}}\right)\left(\frac{a}{1 \mathrm{AU}}\right)^{-1 / 2}\left(\frac{1}{\sqrt{1-e^{2}}}\right)\left(\frac{M_{\star}}{M_{\odot}}\right)^{-1 / 2}
$$

where $M_{\star}$ and $M_{\mathrm{p}}$ are the stellar and the planetary masses, $M_{\odot}$ and $M_{\oplus}$ are the solar and Earth's masses, $a$ is the semimajor axis, and $e$ is the eccentricity. The RV variation of G-type stars orbited by an Earth twin, of the order of $10 \mathrm{~cm} / \mathrm{s}$, is challenged by the "jitter" of the stellar RV originating from magnetic activity, convection, and so on (e.g., Saar et al., 1998, Queloz et al., 2001; Dumusque et al., 2011a, 2011b; Boisse et al., 2012), as well as instrumental noise (see Fischer et al., 2016, for a recent overview of the field). However, the RV amplitude of a late-type star orbited by a HZ Earth-sized planet is larger due to the small stellar mass $\left(M_{\star} \lesssim 0.5 M_{\odot}\right)$ and the small orbital distance of the HZs $(a \lesssim 0.3 A U)$. Such planets have already been discovered through the RV method (Anglada-Escudé et al., 2012, 2016), and the ongoing development of high-resolution spectroscopic instruments will further enforce the discoveries of similar targets (Section 3.1.2). Near-future transit survey by TESS will focus on those around nearby late-type stars, providing a synergy with RV observations. While temperate Earth-sized planets around solar-type stars typically suffer from the observational noise, those around bright and quiet stars and/or those on the larger end may become observable. In addition, RV observations will be facilitated once the planetary signal is detected by other means (e.g., transit sig- nals obtained with PLATO; Section 3.1.2) because the priors for the orbital period and ephemeris will ensure the efficient use of telescopes as well as make the data reduction easier.

If the target planet is in a multiplanet system and the planetary companion (or companions) is (are) observed to transit, planetary mass can also be constrained from the TTVs as a result of a mass-dependent dynamical perturbation of the planet (Agol et al., 2005; Holman and Murray, 2005). This method is based on photometric data with high time resolution and is useful when the host star's RV modulation is difficult to observe. Indeed, the masses of some of the recently discovered Earth-sized planets have been constrained through this method (e.g., Lissauer et al., 2011; Gillon et al., 2017). TTV may be used to constrain the masses of temperate Earth-sized transiting planets around solar-type stars as targeted by PLATO where RV methods could be confounded.

Interior composition. Once both radius and mass are measured, internal structure may be constrained. The massradius relationship allows us to distinguish rocky/metallic terrestrial planets with thin atmospheres from planets with thick atmospheric envelopes, and perhaps water-rich ones (e.g., Léger et al., 2004), although the intermediate densities are confronted by degeneracies (e.g., Fortney et al., 2007; Seager et al., 2007; Rogers and Seager, 2010). Combined with stellar composition, inferences on the compositions of rocky material could also be made (Dorn et al., 2017a, 2017b).

Orbital elements. The orbital parameters most relevant to the habitability discussion are semimajor axis and eccentricity, which determine the incident flux and its time variation. The semimajor axis is constrained from the periodicity of transit light curves provided that the stellar mass is known, while eccentricity can constrained from RV observations, TTV, and/or occasionally the requirement for stability of the system for multiplanetary systems (e.g., Barnes and Quinn, 2001). (The close-in planets are, however, often assumed to be in a circular orbit due to tidal effects.)

Knowing the orbital ephemerides to high precision is essential for the efficient use of telescopes for follow-up observations of known exoplanetary systems as well as for the search of transit signals of RV-detected planets. It has been shown that uncertainties in the eccentricity and argument of periastron can lead to large errors in transit time calculations (Kane and von Braun, 2008; Kane et al., 2009). The major issue arises from the uncertainties in the orbital period and time of periastron passage, as well as the time elapsed since the most recent data was acquired, because these cause a drift in phase. In most cases, only a handful of additional RV measurements is needed to provide a dramatic improvement in orbital period and re-sync the location of the planet in its orbit, provided the observations are acquired with the same telescope and instrumentation to remove the need for a data offset.

3.1.2. Opportunities through 2030. From space, the repurposed Kepler spacecraft, renamed K2, is currently under operation and observing 14 fields near the ecliptic plane in turn to find more transiting planets. $\mathrm{K} 2$ is planned to continue its observations until 2018. There are also groundbased surveys of transiting planets specifically targeting Earth-sized planets around late-type stars, including MEarth 
(Charbonneau et al., 2009; Berta-Thompson et al., 2015) and TRAPPIST (Gillon et al., 2016, 2017).

The primary contributor to the mass measurements of transiting planets is RV observations with ground-based telescopes. In the coming years, a new set of stable highresolution spectrographs in the visible and near-IR will be commissioned at $10 \mathrm{~m}$ class telescopes and smaller. The Infrared Doppler instrument (IRD: Y, J, and $\mathrm{H}$ bands) for the Subaru Telescope, which adopts laser frequency combs as wavelength calibration to enable extremely high RV precisions, will start operating in 2018 and will be the first ultra-stable spectrograph in the near-IR range (Tamura et al., 2012). A precursor, the CARMENES spectrograph (optical, Y, J, and $\mathrm{H}$ bands), started operating at the Calar Alto $3.5 \mathrm{~m}$ telescope in 2016 (Quirrenbach et al., 2016). Two other near-IR laser-frequency comb-based spectrographs, the Habitable Planet Finder (HPF: Y and J bands) for the $9.2 \mathrm{~m}$ Hobby-Eberly Telescope (Mahadevan et al., 2012) and SPIRou (Y, J, H, and K bands) for the $3.6 \mathrm{~m}$ Canada-France-Hawaii Telescope (Delfosse et al., 2013), will be ready for use in 2017. Beyond 2018, high-precision spectrographs for planet surveys will be commissioned: CRIRES plus for the Very Large Telescope (VLT; Follert et al., 2014; Dorn et al., 2016), iLocater for the Large Binocular Telescope (LBT; Crepp et al., 2016), Near InfraRed Planet Searcher (NIRPS) as a near-IR version of the High Accuracy Radial velocity Planet Searcher (HARPS) for the $3.6 \mathrm{~m}$ telescope at La Silla Observatory, and the Echelle SPectrograph for Rocky Exoplanet and Stable Spectroscopic Observations (ESPRESSO) with VLT, which is the first spectrograph designed with the goal of reaching $20 \mathrm{~cm} / \mathrm{s}$ for its overall RV precision (Pepe et al., 2014). Eventually, the measurements made by such instruments will be limited by noise imparted by our own atmosphere. If this proves a limiting factor in the detection of Earth-sized planets around Sun-sized stars, then such detections will have to be made from space.

In 2018, TESS and CHEOPS will be launched to discover nearby transiting planets, with the primary targets being short ( $<30$ day) orbit planets, including HZ planets around late-type stars.

The Transiting Exoplanet Survey Satellite (TESS) (Ricker et al., 2014) is an all-sky, 2-year Explorer-class planet-finder mission launched in 2018, designed to identify planets ranging from Earth-sized planets to gas giants, covering a wide range of stellar types and orbital distances. The main goal of the TESS mission is to detect (small) planets around bright host stars that will be good targets for atmospheric characterization with, for example, JWST. TESS will tile the sky with 26 observation sectors, spending at least 27 days staring at each $24^{\circ} \times 96^{\circ}$ sector and observing 200,000 stars, as defined in the TESS Input Catalog (TIC). The sectors will overlap at the ecliptic poles, covering the JWST continuous viewing zone (CVZ), in order to search for smaller- and longer-period planets. It was shown that TESS will find approximately 1700 transiting planets from its 200,000 preselected target stars-based on simulations of the nearby population of stars, occurrence rates of planets from the Kepler mission, models of photometric performance, and sky coverage of the TESS cameras (Sullivan et al., 2015). Sullivan et al. (2015) also predicted that TESS will detect approximately 48 planets with $R_{p}<2 R_{\oplus}$ and $0.2<S_{p} / S_{\oplus}<2\left(R_{p}\right.$ and $S_{p}$ are the radius and the in- cident flux of the planet, respectively, and $\mathrm{R}_{\oplus}$ and $\mathrm{S}_{\oplus}$ denote Earth's values) around late-type stars with effective temperature lower than $4000 \mathrm{~K}$. Between 2 and 7 of these planets will have host stars brighter than K-band magnitude of 9 and will be very interesting targets for JWST to follow up by spectrophotometrically characterizing their atmospheres and searching for the first signs of habitability (Sections 3.2 and 3.3).

The CHaracterising ExOPlanet Satellite (CHEOPS) mission (European Space Agency CEOPS Definition Study Report (2013); Beck et al., 2016) is the first ESA Small (Sclass) mission to perform ultrahigh precision photometry of exoplanetary systems. Its main objective will be to search for transits around bright stars known to harbor planets detected via $\mathrm{RV}$ measurements. It will aim to determine both whether the known planets transit or not and the transit detection of additional close-in planets not detected by RV. This search will focus on shallow transits on bright stars $(6<V<9$ mag, where $V$ is the $\mathrm{V}$-band magnitude) in the mass range smaller than Neptune with orbital periods of up to $\sim 50$ days. When a transit is found, it provides the unique capability of determining radii and therefore densities with $\sim 10 \%$ accuracy for these targets. Using the density provided by CHEOPS, one can infer the atmospheric volume in a wide parameter space of environmental conditions. CHEOPS will also provide improved radii for already known planets and planets that will be discovered by the future space-based or ground-based transit surveys. This sample of well-characterized small transiting exoplanets around bright host stars will be a group of targets very well suited for upcoming space-based and ground-based platforms, which focus on spectroscopic characterization of exoplanetary atmospheres.

In the 2020s, transiting planets in a broader parameter space will be surveyed by PLAnetary Transits and Oscillations of stars (PLATO) (Rauer et al., 2014). PLATO has been selected for the M3 launch opportunity (currently planned for 2026) in ESA's Cosmic Vision 2015-2025 program. PLATO's main science goal is to photometrically detect planetary transits and to characterize exoplanets and their host stars, including terrestrial planets in the HZs of solar-type stars, by monitoring up to one million stars covering up to $50 \%$ of the sky. Extensive end-to-end simulations have shown that PLATO will be able to detect Solar System analogues: the discovery of Venus and Earth analogues transiting G-type stars like our Sun is feasible (Hippke and Angerhausen, 2015; PLATO Definition Study Report (2017)). Characterization includes the following goals for the uncertainties: $3 \%$ for planetary radii, $10 \%$ for planetary masses (through RV measurements and TTVs), and $10 \%$ for planetary system ages (via asteroseismology of host stars), for planets orbiting bright stars. The resulting large sample of accurately characterized terrestrial planets at orbital periods beyond 3 months will be a unique contribution of PLATO to exoplanet research and allow for comparative exo-planetology up to 1 AU orbital distance. Planets orbiting the brightest stars will be key targets for transit spectroscopy of their atmospheres with telescopes such as JWST or the ELTs. Radius measurements of individual planets as well as the statistical mass-radius relationship of terrestrial planets at a larger orbital separation found by PLATO also serve as a basis in characterizing such targets with future direct-imaging missions, where the 
planetary size is difficult to measure directly. In addition to these exoplanet studies, the large data set of stellar light curves obtained by PLATO will allow us to study the stellar structure, evolution, and activity through asteroseismology and rotational modulations, which provide additional science returns into stellar, galactic, and extragalactic research.

\subsection{Chemical/Climatological characterization: Transmission spectroscopy}

3.2.1. Method and sensitivity. Transmission spectroscopy is a technique to detect the difference between out-oftransit and in-transit spectra, which can reveal the absorption and scattering properties of planetary atmospheres (Seager and Sasselov, 2000). Figure 3 is the transmission spectrum of Earth observed using lunar eclipse (Pallé et al., 2009), exhibiting the major absorption features of $\mathrm{H}_{2} \mathrm{O}, \mathrm{O}_{2}, \mathrm{O}_{3}, \mathrm{CO}_{2}$, and $\mathrm{CH}_{4}$, imposed on a slope due to Rayleigh scattering.

The strength of the spectral features relative to the total stellar flux is estimated by

$$
S \sim \frac{2 N_{H} H R_{\mathrm{p}}}{R_{\star}^{2}} \sim 84 \mathrm{ppm}\left(\frac{N_{H}}{4}\right)\left(\frac{H}{8 \mathrm{~km}}\right)\left(\frac{R_{\mathrm{p}}}{R_{\oplus}}\right)\left(\frac{R_{\star}}{0.1 R_{\odot}}\right)^{-2}
$$

where

$H=\frac{\mathcal{R} T}{\mu_{\mathrm{atm}} g} \sim 7.6 \mathrm{~km}\left(\frac{T}{250 \mathrm{~K}}\right)\left(\frac{R_{\mathrm{p}}}{R_{\oplus}}\right)^{2}\left(\frac{M_{\mathrm{p}}}{M_{\oplus}}\right)^{-1}\left(\frac{\mu_{\mathrm{atm}}}{28 \mathrm{~g} / \mathrm{mol}}\right)^{-1}$

while $R_{\star}$ and $R_{\mathrm{p}}$ are the stellar and planetary radii, respectively, $\mathcal{R}$ is the gas constant, $T$ is atmospheric temperature, $\mu_{\mathrm{atm}}$ is the mean molecular mass of the atmosphere, and $g$ is the surface gravity of the planet. Here, the depth of spectral features is represented by $N_{H} H$, where $H$ is the scale height of the atmosphere and $N_{H}$ is a factor, which is typically 1-5 for spectral features in the optical to far-IR range with spectral resolution $\mathbb{R}=100-1000$, depending on the atmospheric composition (e.g., Kaltenegger and Traub, 2009). In Eq. 2, we normalized the signal for an Earth-sized planet with an Earthlike $\mathrm{N}_{2}$-dominated atmosphere around a late M-type star with $R_{\star} \sim 0.1 R_{\odot}$, similar to TRAPPIST-1 (an M8 star; Gillon et al., 2016). If the host star has the solar radius and other things are equal, the signal would be less than $1 \mathrm{ppm}$, too small to be detectable in the near future as described below. However, planetary parameters including atmospheric mean molecular mass and the surface gravity vary signal levels.

The detectability of the features depends on the observational strategies, instruments used, and the analysis processes. In an idealized case where one tries to identify a spectral feature in a continuum whose only noise source is the photon noise, the signal-to-noise ratio (SNR) is determined by the stellar photon counts $N_{\star}$ and the signal level $S$ :

$$
\begin{aligned}
& \mathrm{SNR} \sim \frac{N_{\star} S}{\sqrt{2 N_{\star}}} \sim \frac{S}{\sqrt{2}} \sqrt{\frac{\pi R_{\star}^{2} \dot{n}\left(\lambda ; T_{\star}\right)}{d^{2}} \pi\left(\frac{D}{2}\right)^{2} \Delta \lambda \Delta t \xi} \\
& \sim 10\left(\frac{N_{H}}{4}\right)\left(\frac{H}{8 \mathrm{~km}}\right)\left(\frac{R_{\mathrm{p}}}{R_{\oplus}}\right)\left(\frac{R_{\star}}{0.1 R_{\odot}}\right)^{-1} \\
& \times\left(\frac{\dot{n}\left(\lambda ; T_{\star}\right)}{\dot{n}(3 \mu \mathrm{m} ; 2500 \mathrm{~K})}\right)^{1 / 2}\left(\frac{d}{10 \mathrm{pc}}\right)^{-1} \\
& \times\left(\frac{D}{6.5 \mathrm{~m}}\right)\left(\frac{\Delta \lambda}{0.1 \mu \mathrm{m}}\right)^{1 / 2}\left(\frac{\Delta t}{30 \mathrm{hr}}\right)^{1 / 2}\left(\frac{\xi}{0.4}\right)^{1 / 2} \\
& \dot{n}(\lambda ; T)=\frac{B(\lambda ; T)}{(h c / \lambda)}=\frac{2 c}{\lambda^{4}} \frac{1}{\exp \left(\frac{h c}{\lambda k_{B} T}\right)-1}
\end{aligned}
$$

where $\lambda$ is the wavelength, $T_{\star}$ is the stellar effective temperature, $B\left(\lambda ; T_{\star}\right)$ is the blackbody radiance as an approximation for the stellar spectrum, $\dot{n}\left(\lambda ; T_{\star}\right)$ represents the corresponding photon count, $d$ is the distance from the star, $D$ is the aperture of the telescope, $\Delta \lambda$ is the wavelength resolution, $\Delta t$ is the integration time through the transits, and $\xi$ is the total throughput. The factor $\sqrt{2}$ comes from the assumption that the in-transit spectrum is calibrated by out-of-transit spectrum with equal integration time; thus, the observation would require $\sim 2 \Delta t$ in total. Again, we adopted stellar parameters similar to TRAPPIST-1, and consider JWST as an example telescope assuming $D=6.5 \mathrm{~m}$ and $\xi=0.4$ (Cowan et al., 2015). Even when considering this idealized situation with planets around late M-type stars, it is likely necessary to accumulate tens to hundreds of hours in total integration time, or tens of transits, in order to detect atmospheric signatures. Such observations can be demanding, and it is therefore critical to have a handful of golden targets that orbit bright host stars and are hence best suited for follow-up observations.

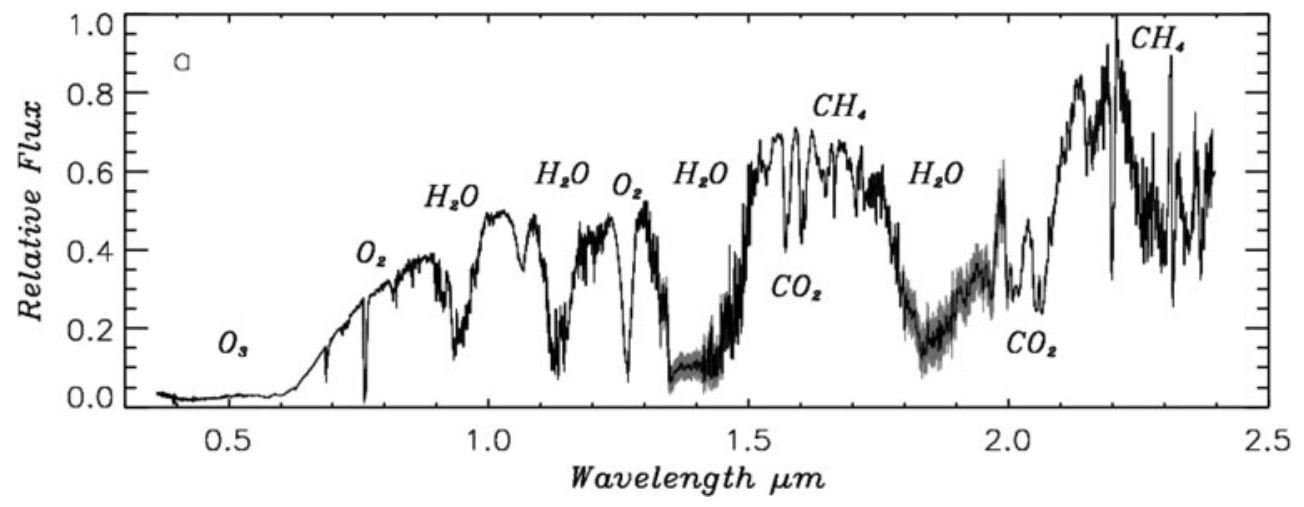

FIG. 3. Transmission spectra of Earth observed at the lunar eclipse, taken from Palle et al. (2009). The spectral resolution is $\mathbb{R} \sim 960$ in the optical and $\mathbb{R} \sim 920$ in the near-IR. 
In reality, there exists additional systematic noise that can be instrumental and/or astrophysical (Barstow et al., 2016; Greene et al., 2016). Currently, HST and Spitzer observations leave tens of ppm as a noise floor that is not reduced after coadding the data. Given that the expected signal level of atmospheric features can be of the order of $10 \mathrm{ppm}$ or less, the detection of these features may be critically challenged by such noise. Signatures of Earth twins around solar-type stars are therefore much less likely to be detected.

While we have assumed low-resolution spectroscopy or multiband photometry above, the past few years have seen fast development in the technique to use high-resolution spectroscopy for characterization of exoplanetary atmospheres. When the resolution is sufficiently high $(\mathbb{R} \gtrsim 100,000$, where $\mathbb{R}$ is the spectral resolution), numerous lines are resolved, and the cross-correlation analysis with the modeled template spectra can be performed (see Fig. 4 for the example of $1.27 \mu \mathrm{m} \mathrm{O}_{2}$ features with varying spectral resolutions). The high-resolution transmission spectroscopy has been successfully performed for the Jupiter-sized close-in planet HD 209458b by Snellen et al. (2010) using CO features. This technique could be applied to characterizing the atmospheres of Earth-sized planets (Snellen et al., 2013; Rodler and López-Morales, 2014). Such highresolution transmission spectroscopy will be a specialty of ground-based telescopes in the coming decade because none of the planned space-based missions can perform high-resolution spectroscopy. Future $30 \mathrm{~m}$ class telescopes will offer powerful facilities suitable for this kind of observation (Section 3.2.3).

\subsubsection{What can be studied?}

Gases. Transmission spectra are sensitive to the constituents of the upper atmospheres (i.e., at low pressures). The SNR favors the wavelengths where the stellar flux peaks but may be observable out to the mid-IR range from space, depending on the instrumental sensitivity, the brightness of the star, and the spectral resolution needed. Major molecular features of Earth's atmosphere in this wavelength range include those from $\mathrm{CO}_{2}(2.7,4.3,15 \mu \mathrm{m}), \mathrm{H}_{2} \mathrm{O}(0.94,1.13$, $1.4,1.9,2.7,6 \mu \mathrm{m}), \mathrm{O}_{2}(0.69,0.76,1.27 \mu \mathrm{m})$, and $\mathrm{O}_{3}(0.5-$

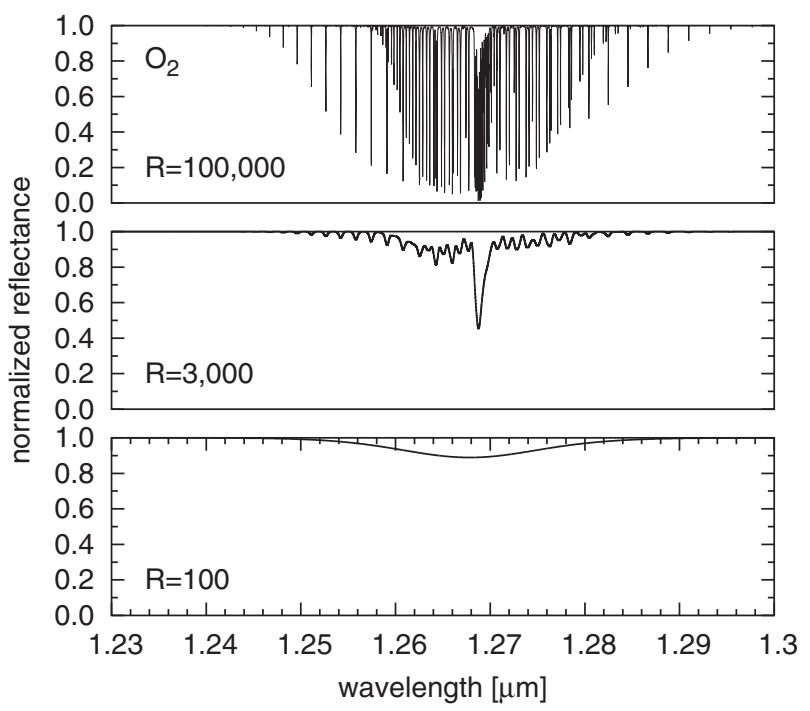

FIG. 4. $\mathrm{O}_{2} 1.27 \mu \mathrm{m}$ features in spectrum of an Earth atmosphere with varying spectral resolution.
0.7, 3.3, 4.7, $9.6 \mu \mathrm{m})$ (e.g., Kaltenegger and Traub, 2009; Bétrémieux and Kaltenegger, 2014; Misra et al., 2014b), while Rayleigh scattering produces the characteristic slope at the short wavelengths. Absorption bands of other molecules, which could potentially be important for other worlds, include $\mathrm{CH}_{4}(2.3,3.3,7.7 \mu \mathrm{m}), \mathrm{CO}(2.35,4.6 \mu \mathrm{m}), \mathrm{SO}_{2}(4.0$, 7.3, 8.6, $18 \mu \mathrm{m}), \mathrm{N}_{2} \mathrm{O}(2.9,3.9,4.5,7.7,17 \mu \mathrm{m}), \mathrm{NH}_{3}(1.5,2$, $2.3,3,6.1,10 \mu \mathrm{m}), \mathrm{O}_{2}-\mathrm{O}_{2}$ dimer features $(1.06,1.27 \mu \mathrm{m})$ $\mathrm{CH}_{3} \mathrm{Cl}(3.4,7,10,14 \mu \mathrm{m})$, and DMS (3.4, 6.9, 7.6, 9.7, $14.5 \mu \mathrm{m})$; see the upper panel of Figure 2 and Catling et al. (2018) for a more comprehensive list. Also, many organic species have absorption bands in the mid-IR, which would help a search for a broader list of potential biogenic molecules (Seager et al., 2016).

The diversity of the atmospheric properties of Earth-sized planets is probably large, and the signal levels will likely vary. In particular, as indicated by Eqs. 2 and 3, planets with hydrogen-rich atmospheres $\left(\mu_{\mathrm{atm}} \sim 2\right)$ should have increased scale heights and hence have amplified signals overall (see, e.g., Miller-Ricci et al., 2009; Pierrehumbert and Gaidos, 2011; Seager et al., 2013; Ramirez and Kaltenegger, 2017). Likewise, surface gravity of the planet affects the overall signal strength through the scale height. Geological activities modify the abundance of the molecules involved in the cycling, such as $\mathrm{CO}_{2}$ and $\mathrm{SO}_{2}$ (Kaltenegger and Sasselov, 2010; Kaltenegger et al., 2010, 2013). Interaction with incoming radiation also matters; for example, in an Earth-like atmosphere, $\mathrm{CH}_{4}$ would accumulate more easily under the UV irradiation of an M-type star than a G-type star, improving the expected signal level of $\mathrm{CH}_{4}$ bands (e.g., Segura et al., 2005; Rauer et al., 2011; Hedelt et al., 2013; Rugheimer et al., 2013, 2015). The mixing ratio of $\mathrm{H}_{2} \mathrm{O}$ also depends on photochemistry (Rauer et al., 2011; Rugheimer et al., 2013, 2015) as well as the effects of three-dimensional atmospheric structures (Fujii et al., 2017a; Kopparapu et al., 2017).

In the inverse problem for determining quantitative estimates of molecular abundances, major molecular absorption depths in transmission spectroscopy constrain the relative abundance of the spectrally active molecules, while the mixing ratios of these species and the spectrally inactive components require the Rayleigh slope unless the higher-order spectral features of absorption bands are measured (Benneke and Seager, 2012; Heng and Kitzmann, 2017).

Transmission spectroscopy in the UV potentially provides a valuable opportunity to probe the extended exospheres of terrestrial planets. When the planetary atomic exosphere is extended, it can absorb a substantial fraction of the stellar emission lines of the same atom during the planetary transit. Indeed, the absorption signatures in the stellar Lyman-alpha emission line $(1215.67 \AA$ ) due to the extended atomic hydrogen tail of the planetary exosphere have been detected for the warm Neptune-mass exoplanet GJ 436b (Ehrenreich et al., 2015; Bourrier et al., 2016), and it may not be a huge leap to observe smaller, cooler planets once good targets are found. A planet with an ocean that evolves to have a significantly moist upper atmosphere due to, for example, the increased intensity of the host star, would lead to efficient hydrogen escape to space, potentially resulting in the similar features (Jura, 2004). In addition, UV transmission spectroscopy may also be used to study of atmospheric molecules, including biosignature candidates such as $\mathrm{O}_{2}, \mathrm{O}_{3}, \mathrm{H}_{2} \mathrm{O}$, $\mathrm{N}_{2} \mathrm{O}, \mathrm{CH}_{4}$, whose cross sections are significantly larger in 
UV than in the visible/near-IR range (Bétrémieux and Kaltenegger, 2013). Such an advantage in the atmospheric molecular signatures, however, is at least partially offset by the generally lower stellar flux available in the UV than in the visible and near-IR range.

Clouds/haze. Cloud/haze layers may be inferred from a broad slope in transmission spectra (e.g., Robinson et al., 2014b) or from muted spectral features (e.g., Kreidberg et al., 2014). Because of the tangential optical paths of transit geometry, even tenuous clouds/haze can contribute to a considerable optical depth, and, if present at low pressure, the molecular features can be significantly weakened. While they can be inconvenient obstacles to detections of molecular features, these may also be seen as a signal that could provide insights into the atmospheric compositions (e.g., $\mathrm{Hu}$ et al., 2013; Checlair et al., 2016) and have even been proposed as potential biosignatures in certain atmospheric contexts (Arney et al., 2016). At longer wavelengths, transmission spectra are less sensitive to high-altitude haze particles due to the reduced extinction efficiency (e.g., $\mathrm{Hu}$ et al., 2013; Arney et al., 2016).

Vertical structure. When the stellar light is transmitted through the planetary atmosphere, it is refracted as a result of the atmospheric density gradient. The refraction has notable effects on transmission spectroscopy (García Muñoz and Pallé, 2011; García Muñoz and Mills, 2012; García Muñoz et al., 2012). Due to refraction, the altitude at which the transmitted (and refracted) ray probes the atmosphere changes over time, and there is a lower limit in altitude (an upper limit in pressure) to which the transmission spectra are sensitive (García Muñoz and Mills, 2012; Garcîa Muñoz et al., 2012, Bétrémieux and Kaltenegger, 2014). Thus, time-resolved transit spectroscopy, while extremely challenging, would in principle probe atmospheric properties at different altitudes (Misra et al., 2014b). In particular, slightly before or after transit, some fraction of the stellar light refracted through the relatively lower part of the planetary atmosphere reaches the observer if the atmosphere is optically thin, producing an increase in the stellar flux. Such an increase may be used to identify an optically thin atmosphere down to the low altitudes, which is favorable for follow-up observations to detect atmospheric molecules of terrestrial planets (Misra et al., 2014b).

Surface pressure, temperature. Transmission spectra could probe the surface pressure if the atmosphere is so thin that atmospheric refraction does not limit our ability to probe the surface layers and radiatively transparent along the slant path at some wavelengths. However, both factors are likely to prevent us from probing the lower atmosphere of Earth-like atmospheres (García Muñoz et al., 2012; Bétrémieux and Kaltenegger, 2014; Misra et al., 2014b); thus, the surface pressure is likely to remain unconstrained. The surface temperature would also likely remain unconstrained, while temperature in the upper atmosphere affects the scale height (Eq. 3).

3.2.3. Opportunities through 2030. So far, HST has been the most powerful observatory for transmission spectroscopy. HST will likely remain the only one capable of observing transmission spectroscopy in the UV in the coming years.
A new space UV observatory from Russia, WSO-UV project, is planned with a $1.7 \mathrm{~m}$ telescope (Sachkov et al., 2014), which can provide deep transit observation capability in the UV.

Transmission spectroscopy in the visible and near-IR has also been performed with ground-based telescopes with varying spectral resolutions. The new stable visible and near-IR high-resolution spectrographs with the $10 \mathrm{~m}$ class telescopes (Section 3.1.2) will be a powerful tool to characterize planetary atmospheres using high-resolution transmission spectroscopy, allowing the search for molecular signatures of hot Jupiters and potentially down to Neptune-sized planets.

In 2020, a new space observatory, the James Webb Space Telescope (JWST), will be launched. JWST is NASA's multipurpose space observatory with a $6.5 \mathrm{~m}$ mirror. One of its main capabilities will be its ability to study the atmospheres of exoplanets with observations in transit, eclipse, or throughout their orbits as a continuous time series to create phase curves. Its halo orbit around the Earth-Sun L2 point allows for long, highly stable, uninterrupted observing sequences compared with ground-based observatories or HST. JWST has four instruments: the Near-Infrared Camera (NIRCam), Near-Infrared Spectrograph (NIRSpec), NearInfrared Imager and Slitless Spectrograph (NIRISS), and Mid-Infrared Instrument (MIRI) over its wavelength range of $0.6-28 \mu \mathrm{m}$ at spectral resolution $\mathbb{R}=4-3250$. In principle, all of these instruments can be used to study transiting exoplanets and will provide a spectrophotometric precision of $10-100 \mathrm{ppm}$ for time series observations spanning from hours to days.

Several studies explored the potential for JWST to observe the targets provided by TESS, CHEOPS, and other surveys by the time of its launch (e.g., Deming et al., 2009; Batalha et al., 2015; Greene et al., 2016), and the transit community already defined an Early Release Science (ERS) case that focuses on testing relevant observing modes to provide the data and expertise to plan the most efficient transiting exoplanet spectrophotometry characterization programs in later cycles (Stevenson et al., 2016). Following these studies, it is anticipated that JWST will enable a survey of $\sim 100$ gas and ice giants and $\sim 10$ s of sub-Neptune-sized planets covering a broad range of spectral types, metallicity, and orbital parameters. These results will advance our understanding of the formation and evolution of these planets (see Section 5.3), as well as the nature of possible highaltitude haze/clouds that suppress the molecular signatures.

JWST will also provide the very first opportunity to characterize the atmospheres of temperate terrestrial planets via transmission spectroscopy, spectroscopy of thermal emission (Section 3.3 below), and the orbital phase curves (Section 4.2 below), but only after co-adding tens of transits, or tens to hundreds of hours in total integration time, depending on the details of the target system (Eq. 4). For a few nearby systems with late-type stars, first investigations of signs of habitability and isolated, inconclusive biomarkers may be possible if the systematic noise of JWST turns out to be sufficiently smaller than the signal levels. For example, Barstow and Irwin (2016) suggested that an Earth-like ozone layer, if it exists, could be detected in 30 transits by JWST for TRAPPIST-1c and TRAPPIST-1d, assuming an Earth-like atmosphere. As an exotic possibility, planets in the HZs of white dwarfs, once detected in the solar 
neighborhood, could constitute other golden targets, having significantly larger signals in transmission spectra; for such targets, even the weaker signature of $\mathrm{O}_{2}$ could be observable after a timeframe as short as several hours of integration (Loeb and Maoz, 2013). For the prospects of eclipse spectroscopy and phase curves of potentially habitable planets with JWST, see Section 3.3 and Section 4.2 below, respectively.

In the 2020s, three 30/40 m class ground telescopes, often called Extremely Large Telescopes (ELTs), are planned to operate. These are the Giant Magellan Telescope (GMT; $24.5 \mathrm{~m}$ diameter), the European-Extremely Large Telescope (E-ELT; $39 \mathrm{~m}$ diameter; now renamed as ELT), and the Thirty-Meter Telescope (TMT; $30 \mathrm{~m}$ diameter), expected to be operational in 2023, 2024, and 2027, respectively. A high-resolution $(\mathbb{R}=25,000-120,000$ spectrograph, GCLEF (Szentgyorgyi et al., 2012), will be installed as one of the first-light instruments on GMT, while other telescopes also contemplate ultra-stable high-resolution spectrographs in their instrumentation plans. The prospects for the detection of molecular features in transmission spectra with such instruments depend on the assumptions of the technical specifications for the proposed instruments and telescopes, and the type of noise sources considered. The $0.76 \mu \mathrm{m}$ oxygen feature of a planet around a nearby $(\sim 5 \mathrm{pc})$ late $\mathrm{M}$ type star could be detected after about 100 transits and 3050 transits with a G-CLEF-like instrument on board GMT and ELT, respectively, supposing the planet possesses an Earth-like atmosphere (Snellen et al., 2013; Rodler and López-Morales, 2014). The nominal specification for the proposed high-resolution instrument (HIRES) for ELT suggested that for TRAPPIST-1b and TRAPPIST-1c, one would be able to detect the $1.3-1.7 \mu \mathrm{m} \mathrm{H}_{2} \mathrm{O}$ band at a SNR of 6 in 2 transits and the $0.9-1.1 \mu \mathrm{m} \mathrm{H}_{2} \mathrm{O}$ band in 4 transits (HIRES team, private communication).
The large apertures of ELTs will improve photometric precision and hence in principle benefit the conventional low-resolution transmission spectroscopy (Pallé et al., 2011). However, this technique relies on the simultaneous observations of nearby bright stars to correct for Earth's atmospheric effects and variability, and the small field of view of ELTs will make it trickier to find suitable comparison stars.

\subsection{Chemical/Climatological characterization: Eclipse spectroscopy}

3.3.1. Method and sensitivity. Dayside emission of transiting planets may be identified using secondary eclipses (planet occultation by the star) by taking the difference between the out-of-eclipse and in-eclipse spectra. Figure 5 shows the simulated thermal emission spectrum of Earth relative to the solar spectrum (black), together with theoretical spectra of Earth-sized planets with Earth-like atmospheres around different spectral types of stars, modeled with 1D photochemical models (Rauer et al., 2011). As indicated in the figure, the contrast between the planetary flux and the stellar flux in the planetary thermal range, $\mathrm{C}_{\mathrm{MIR}}$, becomes on the order of $1-100 \mathrm{ppm}$ at $\geq 8 \mu \mathrm{m}$ for temperate Earth-sized planets around late-type stars. In this range, features of $\mathrm{H}_{2} \mathrm{O}(5-8 \mu \mathrm{m}) \mathrm{CH}_{4}$ $(7.7 \mu \mathrm{m}), \mathrm{O}_{3}(9.6 \mu \mathrm{m})$, and $\mathrm{CO}_{2}(15 \mu \mathrm{m})$ are seen. For those around G-type stars, $C_{\mathrm{MIR}} \lesssim 1 \mathrm{ppm}$, easily overwhelmed by the expected noise floor. An estimate of the contrast in the thermal regime is given by:

$$
\begin{gathered}
C_{\mathrm{MIR}}(\lambda) \sim 54 \mathrm{ppm}\left(\frac{R_{\mathrm{p}}}{R_{\oplus}}\right)^{2}\left(\frac{B\left(\lambda ; T_{\mathrm{p}}\right)}{B(10 \mu \mathrm{m} ; 300 \mathrm{~K})}\right) \\
\left(\frac{R_{\star}}{0.1 R_{\odot}}\right)^{-2}\left(\frac{B\left(\lambda ; T_{\star}\right)}{B(10 \mu \mathrm{m} ; 2500 \mathrm{~K})}\right)^{-1},
\end{gathered}
$$
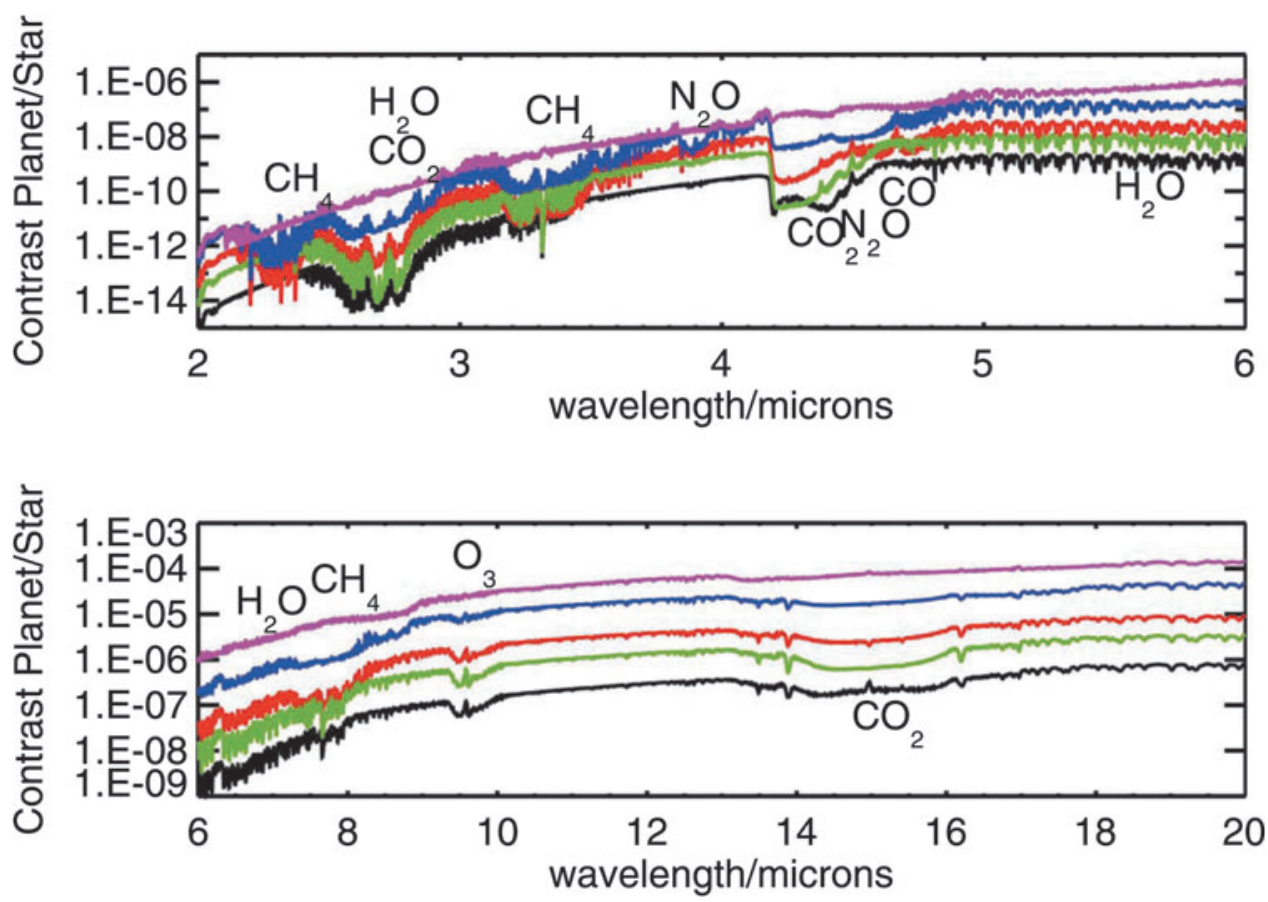

FIG. 5. Modeled thermal emission spectra of cloud-free Earth-like planets around the Sun (black), AD Leo (red), an M0 star (green), an M5 star (blue), and an M7 star (magenta), taken from Rauer et al. (2011). Reproduced with permission (C) ESO. 
Assuming an idealized photon-noise-limited situation with low-resolution spectroscopy, the SNR can be expressed as

$$
\begin{aligned}
& \mathrm{SNR} \sim \frac{N_{\star} C_{\mathrm{MIR}} \delta}{\sqrt{2 N_{\star}}} \sim 1.6 \delta\left(\frac{R_{\mathrm{p}}}{R_{\oplus}}\right)^{2}\left(\frac{\dot{n}\left(\lambda ; T_{\mathrm{p}}\right)}{\dot{n}(10 \mu \mathrm{m} ; 300 \mathrm{~K})}\right) \\
& \quad \times\left(\frac{R_{\star}}{0.1 R_{\odot}}\right)^{-1}\left(\frac{\dot{n}\left(\lambda ; T_{\star}\right)}{\dot{n}(10 \mu \mathrm{m} ; 2500 \mathrm{~K})}\right)^{-1 / 2}\left(\frac{d}{10 \mathrm{pc}}\right)^{-1} \\
& \quad \times\left(\frac{D}{6.5 \mathrm{~m}}\right)\left(\frac{\Delta \lambda}{0.1 \mu \mathrm{m}}\right)^{1 / 2}\left(\frac{\Delta t}{30 \mathrm{hr}}\right)^{1 / 2}\left(\frac{\xi}{0.4}\right)^{1 / 2}
\end{aligned}
$$

where $\delta$ is the relative depth of the spectral features. Again, the fiducial values for the parameters mimic TRAPPIST-1, a late M-type star. We may consider lower wavelength resolution (i.e., larger $\Delta \lambda$ ) as the spectral features are typically broader in the mid-IR. Still, depending on the configuration, eclipse spectroscopy is as demanding as transmission spectroscopy. Eclipse spectroscopy will not be feasible for planets around solar-type stars nor in the visible/near-IR range, where the contrast between the star and the planet is smaller than $1 \mathrm{ppm}$ (Eq. 11 below).

\subsubsection{What can be studied?}

Gases and thermal profile. Eclipse spectroscopy works best around $8-30 \mu \mathrm{m}$ where the planet-to-star contrast is large while the planets are not too faint. Signatures of major small molecules in this range include $\mathrm{O}_{3}(8.9,9.6,14 \mu \mathrm{m}), \mathrm{CO}_{2}(15 \mu \mathrm{m})$, $\mathrm{CH}_{4}(7.7 \mu \mathrm{m}), \mathrm{SO}_{2}(8.6,18 \mu \mathrm{m})$ and $\mathrm{N}_{2} \mathrm{O}(7.7,8.6,17 \mu \mathrm{m})$; see Figure 2. In addition, volatile organic compounds produced through biological processes also have absorption bands (e.g., Domagal-Goldman et al., 2011). Compared with transmission spectrum, emergent thermal emission can probe deeper atmosphere due to the short optical path length and is less likely to be obstructed by tenuous haze layers.

The molecular features in thermal emission depend not only on the abundance of molecules but also upon the temperature profile of the atmosphere. The decreasing temperature as a function of altitude results in absorption features, while thermal inversion layers can create emission features. If the planet does not have a strong vertical temperature gradient, molecular features are weakened. Thus, some information of vertical temperature gradient can be obtained. Once the detailed features of line shapes could be resolved, which is unlikely through 2030, they would further constrain the vertical temperature profiles. If the atmosphere is optically thin at some thermal wavelengths, thermal emission spectra can constrain the surface temperature, one of the key factors for habitability.

While thermal emission spectra are sensitive to these properties, the interpretation as an inverse problem may not be straightforward (for a retrieval study for a cloud-free Earth-like atmosphere, see von Paris et al., 2013). The presence of clouds and the three-dimensional heterogeneity further complicates the problem. The full retrieval would require sophisticated parametric models of atmospheres as well as high-precision observations to feed into the models.

The ingress and egress light curves of the planetary eclipse have offered the opportunity to obtain 2D maps of the dayside of hot Jupiters (de Wit et al., 2012; Majeau et al., 2012). Applying to Earth-sized planets, however, would be exceedingly difficult due to the weakness of the planetary signal.
Solid surface. If the atmosphere is optically thin, we will see the spectroscopic features of the surface rocky materials. Notable features in the mid-IR include bands from Si-O bonds of rocky materials around 10 and $20 \mu \mathrm{m}$ (Hu et al., 2012a; Fig. 2).

3.3.3. Opportunities through 2030. Since the eclipse spectroscopy of potentially habitable planets favors the midIR observations, space observatories work best. So far, most eclipse spectrophotometry of Jupiter-like planets has been performed with the Spitzer space telescope. In the near future, JWST (Section 3.2.3) will be the most promising observatory. However, a smaller-than-predicted noise floor would be necessary to detect spectral features in the thermal emission through eclipse observations.

\section{Characterizing Planets with General Orbital Inclination}

In this section, the methods to characterize potentially habitable planets with general orbital inclination are considered. While non-transiting planets are missed by the observation techniques unique to transiting ones, they are in general closer to the Earth, benefiting other follow-up observations. In the following, astrophysical characterization of nontransiting planets (Section 4.1), chemical, climatological characterization through phase curve measurement (Section 4.2), high-contrast imaging (Section 4.3), and the spectral method (Section 4.4) are discussed. The format is similar to that of Section 3.

\subsection{Astrophysical characterization}

4.1.1. Methods and sensitivity. Radius. Although radius is one of the very basic properties of planets, radii of nontransiting planets are difficult to measure directly in the foreseeable future. When planets are imaged in the visible to near-IR range where the scattered light dominates, the disk-integrated intensity is essentially proportional to squared radius times planetary albedo (Eq. 11 below), so in general the radius is degenerate with albedo. For synchronously rotating atmosphere-free planets, planetary radii (as well as albedo) may be estimated from the phase curves (Maurin et al., 2012), although such planets will not be regarded as potentially habitable. Disk-integrated spectra in the mid-IR (i.e., thermal emission) could better constrain planetary radius (e.g., Des Marais et al., 2002), but currently there are no projects that are capable of such observations (Section 6.2.1).

Mass. Planetary mass can be estimated through the RV method, as in the case of transiting planets. Without another type of observation, however, the degeneracy between the planetary mass and the inclination angle cannot be disentangled (Eq. 1), while statistically the expected value of the true planetary mass is $4 / \pi$ of the measured value for $m_{p} \sin i$. The true mass can be obtained if the inclination is constrained from other types of observation, for example, multi-epoch direct-imaging observations (Section 4.3) or the Doppler shift of the planetary spectra (Section 4.4). Regarding the sensitivity to HZ Earth-sized planets, the same argument holds as described in Section 3.1: those around solar-type stars are more challenging, while those around nearby latetype stars will probably be accessible. 
Another potential probe of planetary mass is astrometry, the method to detect the reflex motion of the star due to the planetary orbital revolution as a periodic movement of the star along the celestial sphere. Astrometry is used by the ongoing Gaia mission to discover large, long-period planets, but Gaia is unlikely to detect temperate Earth-sized planets (Perryman et al., 2014). The capability of astrometry detecting temperate Earth-sized planets is being discussed in the context of the LUVOIR-type far-future mission concept (Section 6.1.2).

Orbital elements. In a similar manner to the case of transiting planets, semimajor axis and eccentricity will be constrained from RV observations if detected. Otherwise, multi-epoch direct-imaging observations (Section 4.3 below) can constrain the orbits.

As discussed in Section 3.1, orbital ephemeris is important for follow-up observations. For direct-imaging observations, the information of orbital ephemeris enables predictions of the timing of the maximum angular separation, and thus accurate ephemerides from RV data will help optimize the use of the precious hours of high-demand space telescopes (Kane, 2013). Orbits of longer-period planets are more difficult to analyze because the uncertainty in the planetary parameters (in particular orbital period and epoch of periastron passage) are large, and data across multiple orbits will substantially improve the estimates.

\subsection{Chemical/Climatological characterization: Phase curves}

4.2.1. Method and sensitivity. Planetary spectra that vary as a function of the star-planet-observer angle (phase angle) may be extracted as a time-varying component of the star + planet spectra, synchronous to the planetary orbital period. In general, variation amplitude is larger for planets whose orbit is closer to edge-on $\left(i \sim 90^{\circ}\right)$ due to the large dynamic range of phase angle along its orbit. Thus, while it can be used for nontransiting planets, transiting planets are the most favorable targets. Phase curve variations would in principle exist both in scattered light and in the thermal emission, but the scattered light of potentially habitable planets is less than $1 \mathrm{ppm}$ of the stellar light (Eq. 11 below) and unfeasible to detect. The thermal emission phase curves of those around latetype stars tend to have the best star-to-planet contrast and are most likely to be detectable. Even for such systems, the contrast is on the order of $10-100 \mathrm{ppm}$ and the phase variation amplitude is smaller than that, so this level of the long-term stability of the stellar radiation and the instruments is a prerequisite for a successful observation. The SNR estimate of thermal emission phase curves in an idealized photon-noiselimited case would be similar to that of secondary eclipse (Eqs. 6 and 7), except for the replacement of $\delta$ by the relative amplitude of phase curves. However, the phase variation itself may be searched for in broadbands (i.e., larger $\Delta \lambda$ ), which can loosen the observational demands.

\subsubsection{What can be studied?}

Heat redistribution - atmosphere/surface flow. The broadband thermal emission phase curves are a useful probe of the thermal redistribution across the globe, which may constrain the potential presence of atmospheres (or perhaps a flow on the surface) (Knutson et al., 2007; Demory et al.,
2016). For example, atmosphere-less planets exhibit strong day-night contrast in thermal emission, which results in a large phase variation amplitude, while planets with thick atmosphere tend to have horizontally more uniform emission temperatures, minimizing the phase variations (e.g., Selsis et al., 2011). The phase curves also depend on the spin state, thermal inertia, and eccentricity. For example, with non-zero thermal inertia, non-synchronously rotating planets exhibit more modest horizontal temperature gradient, hence smaller phase variations, than synchronously rotating planets (e.g., Selsis et al., 2013).

Clouds. Thermal phase curves are affected by the largescale cloud patterns if they exist. Interestingly, synchronously rotating planets covered with oceans tend to develop thick clouds in the substellar region and produce characteristic patterns in the orbital phase curves when highly irradiated (Yang et al., 2013; see also Hu and Yang, 2014, for a more realistic treatment of ocean dynamics); this could even indirectly imply the underlying surface liquid water.

Gases. Spectrally resolved phase curves, or "variation spectra" (Selsis et al., 2011), imprint the signatures of atmospheric molecules because their wavelength-dependent opacity changes the pressure level at which the phase curves probe, and the different pressure levels may have different horizontal patterns of temperature (e.g., Stevenson et al., 2014). The list of the potential target molecules is similar to that for thermal emission eclipse spectroscopy (see Section 3.3.2).

4.2.3. Opportunities through 2030. As in the case of eclipse spectroscopy, JWST is a promising observatory for thermal emission phase curves of exoplanets, potentially down to temperate Earth-sized planets around nearby latetype stars. The thermal phase variation amplitude of Proxima Centauri $b$ is estimated to be on the order of $10 \mathrm{ppm}$ or less if it possesses an Earth-like atmosphere, and 100 ppm if it is atmosphere-less, assuming 60-degree inclination (e.g., Kreidberg and Loeb, 2016; Turbet et al., 2016: Boutle et al., 2017). These variations could be observable with the Low-Resolution Spectrograph (LRS) mode of MIRI onboard JWST, depending on the noise floor and the stability of the stellar radiation, and could provide the clues to the potential presence of an atmosphere.

\subsection{Chemical/Climatological characterization: High-contrast imaging}

4.3.1. Method and sensitivity. Direct imaging allows access to exoplanets at all orbital inclinations, thus offering the only path to characterizing the full suite of exoplanets in the solar neighborhood. However, direct imaging of exoplanets separated from the host star is greatly complicated by stellar glare and must rely on instruments to block the onaxis light from target stars while redirecting the effects of diffraction. Coronagraphs and starshades are currently two possible starlight suppression approaches. The former is placed within the payload of a space telescope (i.e., an "internal" occulter), whereas the latter is its own spacecraft positioned many tens of thousands of kilometers away from a space telescope (hence an "external" occulter).

For both methods, the smallest angular separation they can probe for the faint planetary signal (inner working angle 


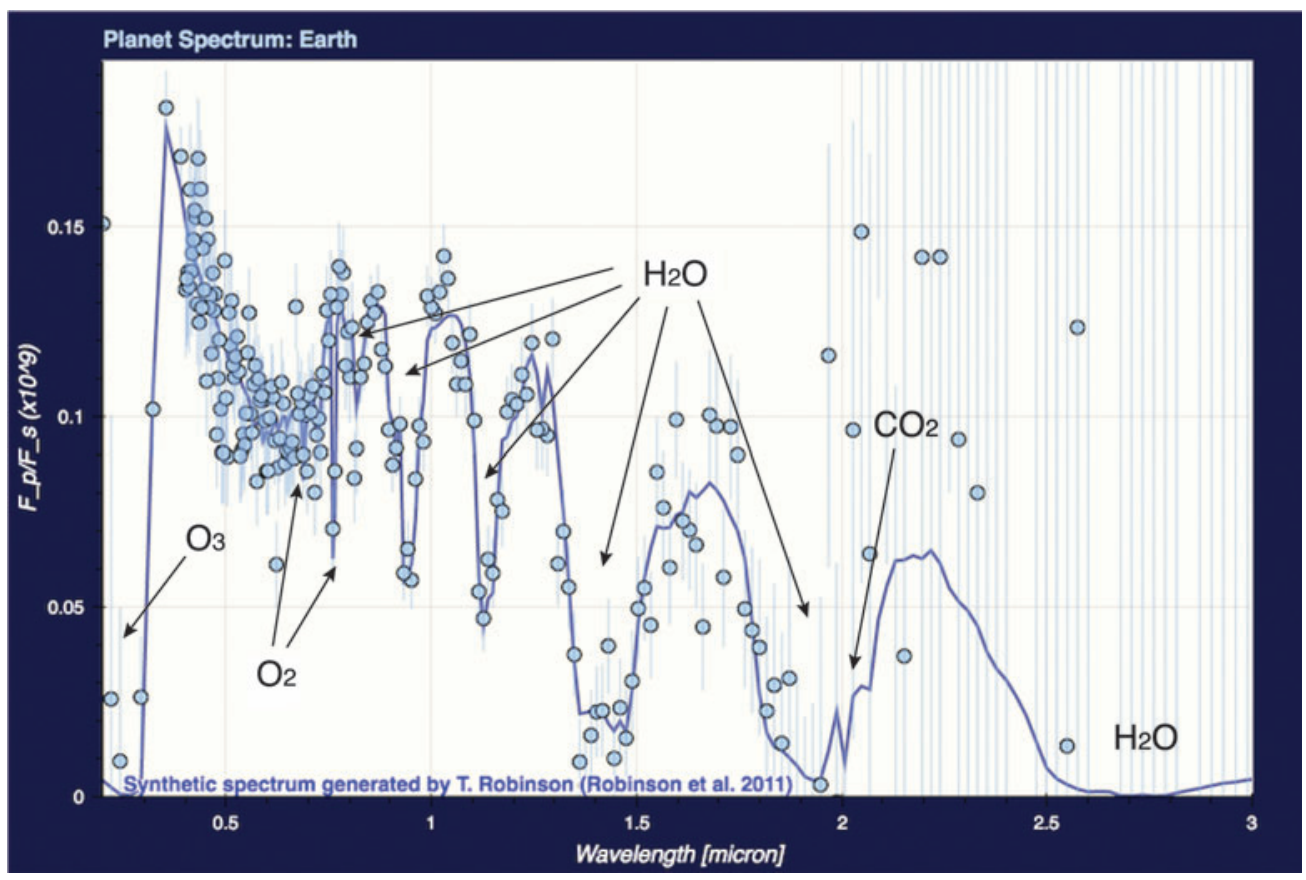

FIG. 6. A modeled scattered light spectrum of Earth (blue solid line) and the mock observation of an Earth twin at 5 pc away assuming a LUVOIR-type telescope with $12 \mathrm{~m}$ diameter and $30 \mathrm{hr}$ of integration time, with resolution $\mathbb{R}=150$ (blue points with error bars), generated at http://jt-astro.science:5106/coron_model. The theoretical line and the noise model are based on Robinson et al. (2011) and Robinson et al. (2016), respectively.

or IWA) should be smaller than the largest angular separation between the planet and the star, $\Delta$, which is:

$$
\Delta=100 \operatorname{mas}\left(\frac{a}{1 \mathrm{AU}}\right)\left(\frac{d}{10 \mathrm{pc}}\right)^{-1}
$$

where "mas" stands for milliarcsecond. The IWA for coronagraphs is expressed by

$$
\mathrm{IWA}=103 \operatorname{mas}\left(\frac{\mathcal{R}}{2}\right)\left(\frac{\lambda}{0.6 \mu \mathrm{m}}\right)\left(\frac{D}{2.4 \mathrm{~m}}\right)^{-1}
$$

where $\mathcal{R}$ is the minimum number of beamwidths between the star and the planet and is a function of the coronagraph design, and ranges from 2 to 4 at present. For starshades, the IWA is approximately

$$
\mathrm{IWA}=\frac{2 F \lambda}{D^{\prime}} \sim 73 \operatorname{mas}\left(\frac{F}{10}\right)\left(\frac{\lambda}{0.6 \mu \mathrm{m}}\right)\left(\frac{D^{\prime}}{34 \mathrm{~m}}\right)^{-1}
$$

where $F$ is the dimensionless Fresnel number (about 10 for $10^{-10}$ contrast) and $D^{\prime}$ is the starshade diameter. These relations mean that, for a fixed-size telescope or starshade, the number of accessible exoplanets at longer wavelengths is greatly reduced relative to the number accessible at shorter wavelengths. Both methods are thus most effective for observations in the visible range where habitable exoplanets are seen in scattered light; the scattered light spectrum of Earth is shown by the solid line in Figure 6 (Robinson et al., 2011).

Both methods must suppress the stellar light to the level of planetary light. The contrast between the scattered light of the planet (at the maximum separation) and the star, $C_{\text {VIS }- \text { NIR }}$, is

$$
C_{\mathrm{VIS}-\mathrm{NIR}} \sim \frac{2}{3 \pi} \frac{R_{\mathrm{p}}^{2}}{a^{2}} A \sim 10^{-10}\left(\frac{R_{\mathrm{p}}}{R_{\oplus}}\right)^{2}\left(\frac{a}{1 \mathrm{AU}}\right)^{-2}\left(\frac{A}{0.3}\right)
$$

where $A$ is planetary albedo. The contrast for temperate planets around late-type stars is improved to $\left(\sim 10^{-9}-10^{-6}\right)$, corresponding to the smaller orbital distance (0.01-0.3 AU), while the angular separation from the star becomes smaller (Eq. 8).

Figure 7 diagrams the contrast and the maximum separation from the host star of the Solar System planets at $10 \mathrm{pc}$ and known exoplanets (points), as well as the performance of the existing and future instruments (lines; detailed below). Ongoing efforts are pushing the sensitivity from upper-right corner toward lower-left, where an Earth-twin resides.

A coronagraph camera has a complex optical train that controls diffraction by using one or more image focal and/or pupil planes to block and beam-shape the on-axis starlight with small specialized masks. Coronagraphs have been used for decades in solar observations, have flown on HST, and will be flown on JWST, and are now used effectively with ground-based telescopes equipped with adaptive optics. The best operational contrast sensitivity achieved to date, from either the ground or from space, is $3 \times 10^{-7}$ at 0.4 arcsec separation by the SPHERE instrument on the VLT observing Sirius (Fig. 7).

In space, a coronagraph with a wavefront sensing and control system, as is now planned for WFIRST, can achieve much higher contrasts (neither HST nor JWST are equipped with this capability). Outside the atmosphere, wavefront 


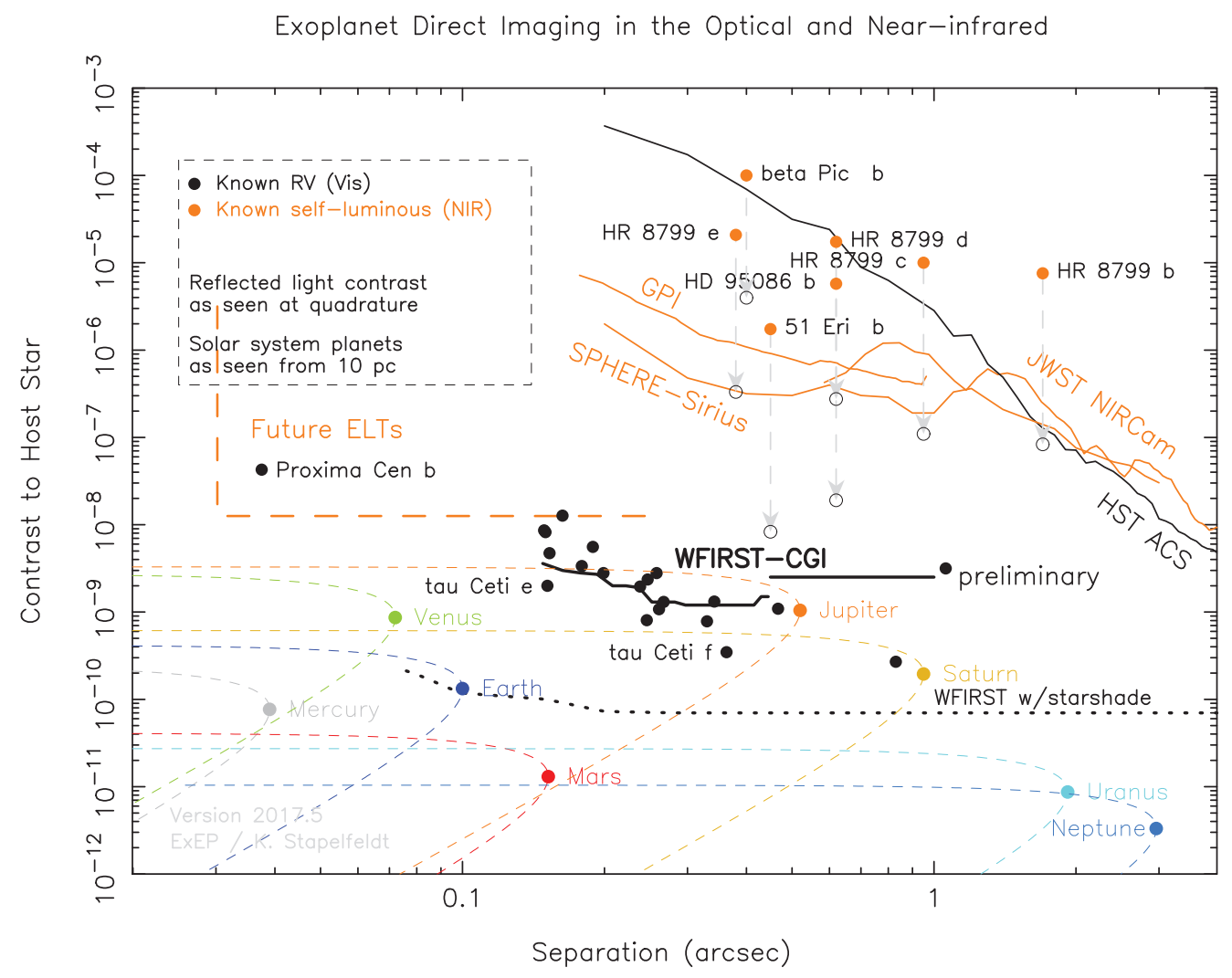

FIG. 7. The star-planet contrast and the star-planet separation of known planetary systems (points), and the performance of existing and future high-contrast imaging instruments (lines). This is the October 2017 version of a plot maintained by the NASA Exoplanet Exploration Program Office. The orange points correspond to the near-IR brightness of known selfluminous directly imaged planets, while the open circles show their theoretical I-band contrast. The black points show the theoretical V-band contrast of planets detected by the RV method. The Solar System planets at $10 \mathrm{pc}$ at the maximum separation are presented in colored points; the dashed lines from these points indicate their orbital phase variations as seen from a direction inclined 30 degrees from the ecliptic. The self-luminous planets detected to date are at contrasts of $10^{-6}$ and brighter, while $10^{-9}$ contrasts are needed to detect Jupiter in scattered light and $10^{-10}$ to detect Earth as seen from outside. The data sources for the instrumental performance lines are as follows: The JWST NIRCam and HST ACS curves were provided by John Krist for Lawson et al. (2012). The GPI curve is for $\mathrm{H}$ band and provided by Bruce Macintosh (personal communication). The SPHERE-Sirius curve is for K band (Vigan et al., 2015, Fig. 2). The 2017 WFIRST CGI curve was provided by deputy instrument scientist Bertrand Mennesson (personal communication). The starshade curve is from Stuart Shaklan (personal communication). The performance curves shown for it are preliminary as of October 2017 and subject to revision.

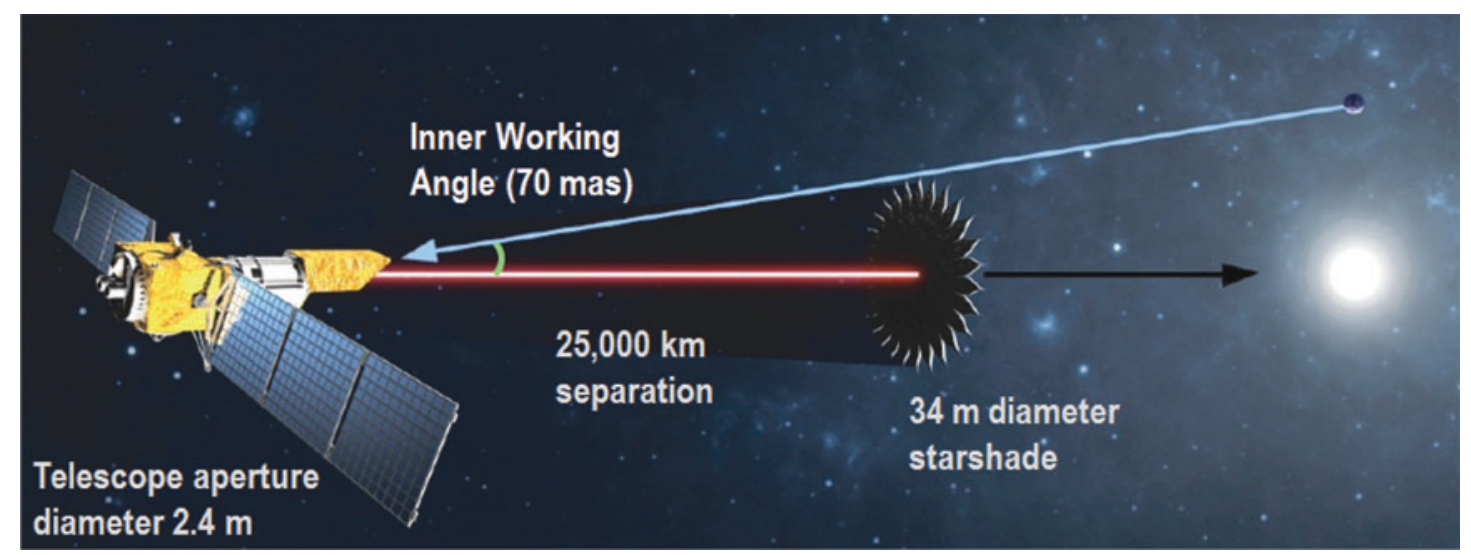

FIG. 8. Flight configuration of a $34 \mathrm{~m}$ starshade in exo-Earth search mode (bandpass of 425-605 nm) was studied as part of the Exo-S Extended Study (https://exoplanets.nasa.gov/internal_resources/225; Seager et al., 2015). This particular configuration attempts to maximize exo-Earth yield and assumes a 3 -year mission; other options considered included both larger and smaller starshades with shorter and longer mission lifetimes. 
correction is much more precise than from the ground, and the system will be stable for hours or days as opposed to the millisecond timescales of atmospheric disturbances. In ground vacuum tests, coronagraphs augmented with wavefront control have already demonstrated $6 \times 10^{-10}$ contrast with a $10 \%$ bandwidth in a $284(\lambda / D)^{2}$ field extending from 3-15 $\lambda / \mathrm{D}$ (Trauger et al., 2013). Work is ongoing to bring the performance to the $1 \times 10^{-10}$ requirement at an IWA of 3 $\lambda / D$ (NASA's Exoplanet Exploration Program Decadal Survey Testbed; N. Siegler, private communication).

A starshade is an external occulter (Fig. 8), and the effects of diffraction are controlled by the precise analytical shape of its petals, and the diffracted light is redirected away from the receiving telescope, creating a dark shadow for which the telescope can fly in formation. Starlight scattered from orbiting planets arrives off-axis, misses the starshade, and can be captured by the telescope (Fig. 8); for a more detailed explanation, see the NASA-chartered Exo-S probe study report (Seager et al., 2015).

Starshades present several key advantages over coronagraphs. First, for a given telescope diameter, a starshade can achieve smaller IWAs (Eq. 10). The smaller IWA enables probing angular regions closer to the targeted stars as well as a larger sample of stellar distances. Second, the achievable contrast ratio is independent of the architecture of the telescope. While segmented and obscured telescope apertures currently pose diffraction challenges for coronagraphs attempting to reach contrast ratios smaller than $10^{-9}$ at visible wavelengths (a topic of active research; NASA's Exoplanet Exploration Program Segmented Coronagraph and Design Analysis study; https://exoplanets.nasa.gov/exep/technology/ TDEM-awards), starshades can in theory create $10^{-10}$ or better dark regions at telescope detectors independent of the aperture architecture, reaching the sensitivity requirement to directly image temperate Earth-sized exoplanets around Sun-like stars. Lastly, starshades can be designed to perform at larger bandwidths and, with fewer optics, significantly higher throughput, thus potentially enabling higher-resolution spectrographs. A drawback, on the other hand, is that the complex positioning of the two spacecraft and re-pointing will take time-approximately 1-2 weeks_-potentially challenging repeat observations for orbit determination and the measurement of seasonal changes and phase angle effects.

The requirement of high-contrast imaging could be loosened when a starlight suppression instrument is combined with spectral separation methods (Section 4.4) (Sparks and Ford, 2002; Riaud and Schneider, 2007; Kawahara et al., 2014; Snellen et al., 2015). Several works (Kawahara and Hirano, 2014; Snellen et al., 2015; Wang et al., 2017) have investigated the potential of the combination of high-contrast imaging instruments and high-resolution $(\mathbb{R} \sim 100,000)$ spectrograph, and showed that molecular signatures of Earth-like planets around late-type stars may be detected with high-contrast imaging instruments that achieve $\sim 10^{-5}$ to $10^{-4}$ contrast (approximately improved by a factor of $10^{3}$ ) when combined with high-resolution spectrograph. This is probably a promising approach to spectral signatures of potentially habitable planets with ground-based observatories. See the next section (Section 4.4) for more discussions.

Another way to increase the detectability of the planet is to utilize polarized light, taking advantage of the fact that stars have low polarizations. Ideally, if the polarization from the star is sufficiently low and the precision of the polarimetry is high, polarimetry alone would allow us to identify the planetary component in the combined star + planet light. Indeed, high-precision polarimetric observations attempted to detect scattered light of hot Jupiter HD 189733 without starlight suppression (Berdyugina et al., 2008, 2011; Wiktorowicz, 2009; Wiktorowicz et al., 2015; Bott et al., 2016); no conclusive detection has been obtained so far, however, while the upper limits of tens of ppm was put. The polarizations of potentially habitable planets are expected to be at an even lower level, and polarization on its own will likely be insufficient for planet detection. Instead, a combination of direct-imaging instruments and polarimetric instruments is a more likely way to utilize this technique.

4.3.2. What can be studied? Once the starlight is sufficiently suppressed, and the exoplanet lies outside of the instrument's IWAs, then the exoplanetary photons can be directly analyzed in the spectral and time domains. It can also provide, in a single observation, the full system context view of multiple exoplanets and zones of dusty debris.

Gases. Spectroscopy of Earth-like planets in the visible to near-IR range shows the molecular signatures including $\mathrm{O}_{2}(0.63,0.69,0.76,1.27 \mu \mathrm{m}), \mathrm{O}_{3}(<0.35,0.5-0.7 \mu \mathrm{m}), \mathrm{H}_{2} \mathrm{O}$ $(0.72,0.82,0.94,1.13,1.4,1.9 \mu \mathrm{m}), \mathrm{CO}_{2}(1.04,1.20,1.43$, 1.6, 2.0, $2.7 \mu \mathrm{m}), \mathrm{CH}_{4}(0.72,0.78,0.88,0.97,1.15,1.4,1.66$, $2.3 \mu \mathrm{m}$ ) (see Fig. 2), while the features at longer wavelengths reduce the number of accessible targets due to the limitation of IWA. A massive $\mathrm{O}_{2}$-dominated atmosphere would produce strong $\mathrm{O}_{2}-\mathrm{O}_{2}$ features $(1.06,1.27 \mu \mathrm{m})$, potentially diagnostic for a false-positive scenario for $\mathrm{O}_{2}$ as a biosignature (Schwieterman et al., 2016). Direct-imaging spectroscopy is more sensitive to the compositions in the lower atmospheres than transmission spectroscopy, and is less affected by tenuous haze layer or high clouds, due to the shorter optical path length.

The spectral resolution $(\mathbb{R})$ required for a detection clearly varies from band to band. For example, assuming an Earth-like atmosphere, $\mathbb{R}>20$ will be necessary to detect $\mathrm{H}_{2} \mathrm{O}$ absorption bands at $0.94 \mu \mathrm{m}$, while the convincing detection of the narrower $\mathrm{O}_{2}$ band at $0.76 \mu \mathrm{m}$ likely requires $\mathbb{R}>100$ (Brandt and Spiegel, 2014). Some scattering and absorption features are broad enough to be captured with fairly low-resolution spectroscopy or multiband photometry. For example, Rayleigh scattering, scattering/absorption by clouds and haze layers broadly affects the wavelength dependence of the scattered light spectra (Hu et al., 2013; Arney et al., 2016; Checlair et al., 2016). Broad absorption features of $\mathrm{O}_{3}$ around $0.6 \mu \mathrm{m}$ may be inferred from the colors of Earth (Krissansen-Totton et al., 2016).

Solid surface. Scattered light spectra include characteristic features of the surface, if the atmosphere is optically thin at least in some areas. Reflectance spectra of commonly seen materials in the Solar System are shown in the lower panel of Figure 2. Spectroscopic features of surface include absorption bands of rocky materials due to charge transfer $(<0.4 \mu \mathrm{m})$ and crystal-field effects (around $1 \mu \mathrm{m})$ and of $\mathrm{O}-\mathrm{H}$ bonds of ice and hydrated materials $(1.5,2,3 \mu \mathrm{m})$, whose exact optical properties depend on the detailed composition and grain size (e.g., Ford et al., 2001; Hu et al., 
2012a; Fujii et al., 2014). Importantly, for the first time, we will be able to access surface reflectance biosignatures such as vegetation's red edge (Ford et al., 2001; Seager et al., 2005; Montañés-Rodríguez et al., 2006; Tinetti et al., 2006; Kiang et al., 2007a, 2007b), "purple edge" (Sanromá et al., 2014), and the peculiar reflectance spectra that occur in a variety of biological pigments of diverse functions (Hegde et al., 2015; Schwieterman et al., 2015), some of which are shown in Figure 2; see Schwieterman et al. (2018) for a comprehensive review. It would be possible that exobiospheres interact with incident radiation to imprint peculiar signatures similar to what we observe on Earth.

Surface liquid bodies. The most essential aspect of climatological characterization for $\mathrm{HZ}$ planets is the presence of an ocean. This can be probed in scattered light through the peculiar anisotropy of scattering by liquid surface, where the reflectivity increases with grazing incident angle (ocean's "glint," Williams and Gaidos, 2008; Oakley and Cash, 2009; Robinson et al., 2010, 2014a, Robinson, 2017). This nature of an ocean exhibits itself as an anomalous increase in planetary albedo at the crescent phase. At such a phase, however, the angular separation between the star and the planet is small, and the scattered light is dark, making direct-imaging observations challenging, possibly requiring the direct-imaging missions beyond 2030.

Planetary albedo. Because the planetary scattered light is proportional to $R_{\mathrm{p}}^{2} A(\lambda)$, the absolute value of planetary albedo $A(\lambda)$ is known in a model-independent manner only when the planetary radius is known from, for example, transit observations, or perhaps from the assumption of interior composition if the mass is known from, for example, RV observations. Planetary albedo would yield the equilibrium temperature, an important reference point for modeling of the temperature profile.

Surface pressure, temperature. A possible indicator of surface pressure in scattered light spectra is the Rayleigh scattering feature. Rayleigh scattering slope essentially depends on the molecular-specific cross section and the column number density of the atmosphere, the latter of which is related to the atmospheric pressure through the surface gravity and the mean molecular weight of the atmosphere. Thus, estimates of these parameters tend to be degenerate. The presence of clouds as well as the wavelength-dependence of surface reflectance can further puzzle the interpretation. The atmospheric pressure also affects the absorption features of molecules. Additional use of signatures of dimers, which vary as the square of the density, may be useful to constrain surface pressure (Misra et al., 2014a). Despite the direct relevance to habitability, surface temperature is difficult to estimate from the scattered light spectra, as it negligibly affects the spectra in this regime (Robinson, 2017).

Spin parameters. Time-resolved direct-imaging observations at the timescale of planetary rotation, if/once they eventually become feasible, potentially provide additional, key dimensions in climatological characterizations. Unless the surface is completely uniform, rotation rate can be measured as a periodicity of the disk-integrated planetary light; rotation rate is one of the fundamental parameters in modeling the climate and habitability of the planets. Earth as a point source changes its albedo by $10-20 \%$ in one rotation (Livengood et al., 2011, also shown in Fig. 9), and Pallé et al. (2008) found that the periodicity can be identified through the autocorrelation analysis despite the variable cloud cover, thanks to the consistent geographic features (e.g., continents). Another important parameter affecting climate, obliquity, could also be inferred through examining the long-term light curves which involve rotational and orbital variations (Kawahara and Fujii, 2010, 2011; Fujii and Kawahara, 2012; Kawahara, 2016; Schwartz et al., 2016). The rotation rate of Jupiter-like planets has been measured by the broadening of the molecular lines using highresolution (Snellen et al., 2014), but similar observations of an Earth-like planet would be exceedingly difficult; the rotation velocity of the Earth is $\sim 26$ times smaller than Jupiter's.

Surface heterogeneity, partial cloud cover. From the rotational variation of scattered light spectra, the heterogeneity of the surface composition may be constrained (e.g., Cowan et al., 2009, 2011; Fujii et al., 2010, 2011, 2017b; Cowan and Strait, 2013). For example, combinations of a liquid surface, particulate rocky materials, and/or snow/ice

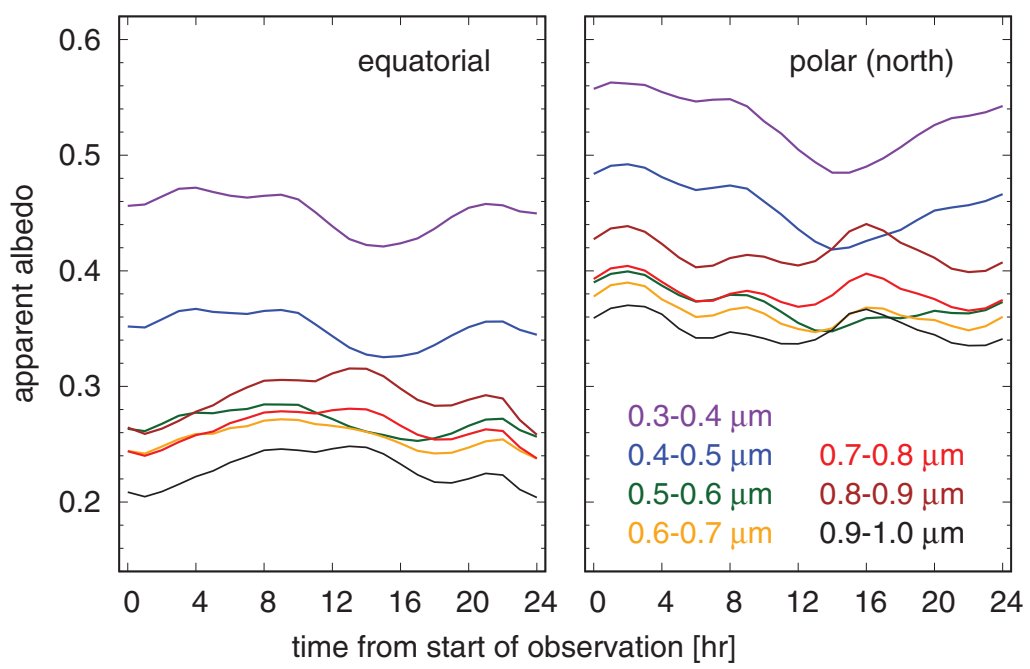

FIG. 9. Seven-band diurnal light curves of the disk-integrated scattered light of Earth, obtained by the EPOXI mission (Cowan et al., 2011; Livengood et al., 2011). Left panel: The equatorial observation started on March 18, 2008 , with phase angle $57^{\circ}$. Right panel: The north-polar observation started on March 27, 2009 , with phase angle $87^{\circ}$. 
contribute to a marked contrast in scattered light spectra at different wavelengths (Figs. 2 and 9). The surface heterogeneity may imply geological processes over the history of the planet, including plate tectonics and volcanic activities (Fujii et al., 2014). The presence of variable clouds could be probed through the deviation of daily light curves from the average light curve (Pallé et al., 2008), or the variability of molecular features (Fujii et al., 2013). Rotational and orbital variations together would even allow us to recover the twodimensional surface map (Kawahara and Fujii, 2010, 2011; Fujii and Kawahara, 2012).

Additional clues from polarization. Once the planet is directly imaged, the polarization of the planetary light may be analyzed when combined with the proper instrument. Polarimetry of Earth includes some interesting features. In theory, Rayleigh scattering by the atmosphere causes a polarization peak near a phase angle 90 degrees, whereas the polarization of reflection by a liquid water surface is the highest at phase angle 106 degrees, and water droplets have their polarization peak at around 30-40 degrees, while multiple scattering reduces the polarization (Stam et al., 2004; McCullough, 2006; Bailey, 2007; Stam, 2008; Williams and Gaidos, 2008; Zugger et al., 2010, 2011; Karalidi and Stam, 2012). Earthshine observations confirmed that the effect of Rayleigh scattering dominates in the disk-integrated polarized light of Earth at short wavelengths (Sterzik et al., 2012; Takahashi et al., 2013), and the polarization decreases to $\sim 9-12 \%$ in the near-IR continuum (Miles-Páez et al., 2014). The "absorption", features of atmospheric molecules, which exhibit themselves as "peaks" in polarized light because of the reduced contribution from multiple scattering (Stam, 2008), are probably as high as 30\% (Miles-Páez et al., 2014). These features potentially provide additional evidence about the planetary surface environment.

4.3.3. Opportunities through 2030. Direct imaging of Jupiter-sized distant planets has been successfully performed with $10 \mathrm{~m}$ class ground-based telescopes, and these observations achieved a contrast of $10^{-4}$ to $10^{-6}$. This level of high-contrast technique could potentially be combined with high-resolution spectrograph to obtain spectral features of smaller planets; we discuss such an approach in Section 4.4. The $30 \mathrm{~m}$ class telescopes (ELTs) to be built in the $2020 \mathrm{~s}$ are also considering coronagraphic instruments, and depending on the performance of such instruments, they might achieve high-contrast imaging of potentially habitable planets even without high-resolution spectroscopy. Their large apertures can make the IWA as small as 10 mas in principle (Eq. 9), which allows us to probe potentially habitable planets around nearby late-type stars. The accessible targets may also include temperate Earth-sized planets around M-type stars in the prolonged pre-main-sequence stage, such as $40 \mathrm{Myr}$ AP Col, 8.4 pc away (Ramirez and Kaltenegger, 2014). Once the habitable planets are detected in high-contrast imaging, an Earth-like $\mathrm{O}_{2}$ absorption feature at $1.27 \mu \mathrm{m}$ would be detectable (Kawahara et al., 2012).

Temperate Earth-sized planets orbiting solar-type stars, however, may never be directly imaged from the ground, due to the $10^{-10}$ contrast requirement, which is limited by Earth's atmosphere and the residual uncorrected wavefront error (Traub and Oppenheimer, 2010, pp 146-147). To achieve such contrast, a space-based observatory will be necessary. Although JWST is equipped with coronagraphic instruments to perform high-contrast imaging of Jupiterlike planets, they will not be able to image Earth-sized planets.

The next space-based opportunity for high-contrast imaging is The Wide Field InfraRed Survey Telescope (WFIRST), which is NASA's next large space observatory with an expected launch date of 2025 and will operate for at least 6 years. Its prime instrument is a 288 megapixel near-IR camera designed to survey the extragalactic sky for dark energy science and to monitor the galactic bulge for gravitational microlensing events. In 2013 the mission was redesigned to use a surplus $2.4 \mathrm{~m}$ telescope and, with the larger aperture, a second instrument was added: an optical coronagraph for exoplanet direct imaging. The coronagraph instrument ("CGI") will be the first space-based demonstration of precision wavefront control that is needed to achieve an image contrast ratio approaching a billion to one at subarcsecond separations. CGI includes both Shaped Pupil and Hybrid Lyot coronagraph masks, a pair of $48 \times 48$ actuator deformable mirrors, and a low-order wavefront sensor to compensate for telescope instabilities. CGI will enable studies of dozens of giant planets in scattered light around stars within $\sim 20 \mathrm{pc}$ of the Sun, using photometry or $\mathbb{R} \sim 50$ spectroscopy over a $0.6-0.95 \mu \mathrm{m}$ bandpass. CGI will not be capable of studying Earth twins, but it will have the contrast sensitivity and angular resolution to study larger planets if they are present in the HZs of half a dozen nearby stars. With luck, WFIRST CGI could obtain the first imaging detection and crude spectroscopy of large temperate terrestrial planets.

While not baselined, a starshade flying in formation with WFIRST (Fig. 8) offers the critical capability of directly imaging terrestrial planets in the $\mathrm{HZ}$ of solar-type stars. The 2015 NASA-chartered starshade probe study was extended to optimize the exoplanet yield of a technology demonstrator WFIRST Starshade Rendezvous mission. The most likely scenario (included in the extended study's final report [https:// exoplanets.nasa.gov/internal_resources/225]), factoring in observational completeness, predicted $28 \mathrm{HZs}$ of solar-type stars could be surveyed by WFIRST for Earth-sized exoplanets with a $34 \mathrm{~m}$ starshade over a 3 -year mission. Assuming a conservative occurrence rate of potentially habitable planets $\left(\eta_{\oplus}\right)$ of $10 \%$, stellar sky brightness of $21 \mathrm{mag} / \mathrm{sq}$ arcsec (zodi and exo-zodi), and an optical throughput of $28 \%$ using the WFIRST CGI optics, 2-3 exo-Earth detections were predicted with a SNR of 6 . Of those, one could have its spectrum taken within a visible bandpass. Assuming $\mathbb{R} \sim 70$, spectral features such as water vapor, oxygen, and potentially even Rayleigh scattering of an exo-Earth atmosphere could be identified. These signatures, along with cloud parameterization and other assumptions, could provide quantitative constraints on oxygen and water vapor mixing ratios along with surface pressure (Feng et al., 2018). Smaller and less expensive "technology demonstrator" starshades $(20 \mathrm{~m})$ with shorter mission lifetimes were also included in the extended study, resulting in only one predicted imaged terrestrial planet and without characterization. A final specific starshade design will be developed over the next couple of years by NASA starshade technology activity managed by the Exoplanet Exploration Program. 


\subsection{Chemical/Climatological characterization: Spectral separation}

4.4.1. Method and sensitivity. Spectral methods may also be used to extract the planetary component from the combined star + planet spectra. In high-resolution spectra $(\mathbb{R} \gtrsim 100,000)$ where individual lines are resolved (Fig. 4), the Doppler-shifted planetary atmospheric lines with respect to the telluric lines and the stellar lines allow one to identify the planetary component, along with the planetary line-ofsight velocity (Snellen et al., 2010). While the planetary signal is buried deeply in the overwhelmingly brighter stellar flux, cross-correlation analysis of the observed spectra with the modeled ones using many lines contributes to an enhanced SNR. Such a technique was successfully demonstrated by the molecular detections of hot Jupiters without using planetary transits, eclipses, or high-contrast imaging. For example, Brogi et al. (2012) detected the CO lines of $\tau$ Boötis $b$ through high-resolution spectroscopy with the Cryogenic Infrared Echelle Spectrograph (CRIRES) at VLT, while de Kok et al. (2013) detected CO lines of HD189733b using the same instruments. The stabilized instruments such as HARPS and HARPS-N have also been used with smaller telescopes $(3.5 \mathrm{~m})$ to make the first detection of the scattered light of hot Jupiter 51 Peg b (Martins et al., 2015).

This method will be limited to ground-based observations in a foreseeable future, as high-resolution spectrographs are not planned to be on a space observatory. However, the contrast between temperate Earth-sized planets and their host stars at the wavelengths observable from the ground $\left(\sim 10^{-10}\right.$ $10^{-6}$; Eq. 11) is much smaller than the successful observations so far $\left(\sim 10^{-3}\right)$. Thus, several works (Sparks and Ford, 2002; Riaud and Schneider, 2007; Kawahara et al., 2014; Snellen et al., 2015; Wang et al., 2017) have proposed the combination of the high-resolution technique with a highcontrast imaging instrument as a viable approach for detecting atmospheric signatures of temperate Earth-sized planets around late-type stars. After suppressing the stellar component to the order of $10^{-5}$ or so, spectral identification of the planetary component will be more feasible.

Recently, Snellen et al. (2017) proposed a related technique where space-based medium-resolution spectroscopy is used to find spectral features of the planetary atmosphere in the combined star + planet spectrum in the mid-IR range where the planet-to-star contrast can be order of 10-100 ppm (for those around late-type stars). While the individual lines are not fully resolved in medium-resolution spectroscopy, the high-frequency features may be identifiable by fitting with theoretical models.

One could also attempt to identify the molecular signatures of planetary atmospheres even in the low-resolution star+ planet spectra, if the molecules should not be present in the stellar atmosphere and thus can be safely attributed to the planetary origin. The success of this method will critically rely on accurately knowing the host star spectrum as well as on the noise floor of the observed data.

4.4.2. What can be studied? Once the Doppler-shifted planetary lines are detected, not only is the presence of targeted molecules revealed, but also the planetary line-ofsight velocity is obtained. This translates to the true mass of the planet and orbital inclination when combined with stellar RV measurements, contributing the astrophysical characterization of the system.

In the visible to near-IR range where the scattered light dominates over thermal emission, the contrast between the planet and the star would indicate $R_{\mathrm{p}}^{2} A$ (Eq. 11). In the midIR regime where the thermal emission dominates, the detailed characteristics of the spectral features can probe on the vertical thermal profile of the atmosphere, as discussed in Section 3.3.

4.4.3. Opportunities through 2030. By combining the ultra-stable high-resolution spectrograph with the highcontrast imaging techniques, the largest existing groundbased telescopes might eventually acquire the potential to access the nearest temperate Earth-sized planets. Lovis et al. (2017) estimated that detection of the scattered light of Proxima Centauri b $(\Delta \sim 37$ mas at the maximum separation) in the visible wavelengths would be possible with the proposed upgrades to SPHERE high-contrast imager combined with ESPRESSO high-resolution spectrograph, after 20-40 nights of total telescope time, assuming an Earth-like atmosphere. Even marginal constraints on $\mathrm{O}_{2}$ may be obtained with an intense use of the telescope.

The ELTs can in principle adopt the same technique. Their larger collecting areas can substantially improve the observational capabilities. For example, it has been estimated that the atmospheric characterization of Proxima Centauri $b$ could be accomplished in about 6 nights using the collecting area of the ELT (HIRES team, private communication). The larger aperture also allows us to probe a greater number of targets with the smaller IWA. Once coronagraphic instruments and high-resolution spectrographs are installed on the ELTs, they will offer promising and unique opportunities for the targets around late-type stars.

In the mid-IR range where potentially habitable planets around late-type stars have the planet-to-star contrast of the order of 10-100 ppm, spectral signatures of the planetary atmosphere could be searched for in the star + planet spectrum even without starlight suppression instruments, if the observations have sufficiently low noise characteristics. Kreidberg and Loeb (2016) estimated that the spectral feature of $\mathrm{O}_{3}$ at $9.6 \mu \mathrm{m}$ of Proxima Centauri b, if present, could be detected in the combined star + planet spectrum after months of observation assuming photon-noise limited precision with a JWST-like telescope. Snellen et al. (2017) suggested that the medium-resolution spectrograph (MRS) mode of MIRI on board JWST has a potential to detect $15 \mu \mathrm{m} \mathrm{CO}$ feature of Proxima Centauri b in 5 days, using their high-frequency characteristics; such observations will require enabling the time-series observations of MIRI/MRS. Clearly, these observations are contingent on a small noise floor.

\section{Contextual Information}

So far, we have focused on the properties of potentially habitable planets themselves. In this section, we discuss how the external contextual information other than the planetary properties may be used to improve the characterization of the planets in question. First, we mention the efforts to characterize the host stars (Section 5.1), as one of the most essential ingredients in assessing the planetary environment. We also consider the information of the architecture 
of the target planetary system (Section 5.2), and the chemical characterization of the gaseous planet (or planets) in the same system (Section 5.3), both of which will be more easily available than the properties of terrestrial planets.

\subsection{Properties of the host star}

5.1.1. Mass, radius, SED in the visible/IR range. Precise measurement of planetary mass and radius depends on accurately knowing the host star, and the planetary climate is largely affected by the spectral energy distribution (SED) in the visible to near-IR range. The basic properties of the host stars such as radius, mass, age, and effective temperature may be estimated based on the observed spectra and the distance (estimated from parallax), or near-IR spectroscopy armed with stellar evolutionary models. Asteroseismology provides additional information to characterize the stellar properties (e.g., Huber et al., 2013), and more asteroseismic data will become available along with searches for transiting planets. In many cases, these basic properties are cataloged with varying accuracy. Depending on the reliability of the model used to derive these values, additional observations of individual host stars may be needed to obtain more precise values for these parameters.

5.1.2. Activity (SED in UV, X-ray, superflares). Photochemical reactions in the planetary atmosphere depend on the SED in the UV range. Photochemistry affects the atmospheric profiles of composition and temperature, influencing the detectability and reliability of some of the biosignatures. For example, model studies (e.g., Segura et al., 2005; Grenfell et al., 2012; Tabataba-Vakili et al., 2016) show that the abundances of $\mathrm{O}_{3}$ and $\mathrm{CH}_{4}$ are sensitive to the UV flux. The UV output may also abiotically produce potential biosignature gases such as $\mathrm{O}_{2}$ (e.g., Hu et al., 2012b; Domagal-Goldman et al., 2014; Tian et al., 2014). In addition, powerful coronal mass ejections, or superflares (Maehara et al., 2012, 2015), would interact with the planetary magnetosphere and cause energetic particles to flood into the atmosphere and induce in situ chemical reactions (Airapetian et al., 2016). Their results suggested that Earth-like $\left(\mathrm{N}_{2}-\mathrm{O}_{2}\right)$ atmospheres would form $\mathrm{N}_{2} \mathrm{O}$, which could then be a false positive for a biosignature. Highenergy radiation toward the X-ray range, called XUV (roughly $1-1000 \AA$ ), drives atmospheric loss through thermal mechanisms (Jeans escape and hydrodynamic escape) and nonthermal mechanisms (e.g., through the charge separation driven by XUV ionization; Airapetian et al., 2017). Thus, these high-energy fluxes over the history of the star critically impact atmospheric evolution.

The strength of stellar high-energy radiation is related to the magnetic activity (stellar dynamo) (e.g., Noyes et al., 1984), and they are negatively correlated with the age and the spin rotation period (e.g., Wilson, 1966; Kraft, 1967; Pallavicini et al., 1981; Wright et al., 2011; Astudillo-Defru et al., 2017a). The profile of $\mathrm{Ca}$ II $\mathrm{H}$ and $\mathrm{K}$ lines is used as an observational proxy of the activity (e.g., Wilson, 1966; Kraft, 1967; Saar and Fischer, 2000; Queloz et al., 2001; Wright et al., 2004), while $\mathrm{H} \alpha$ line is also becoming more widely used as an activity tracer for low-mass stars (e.g., West et al., 2008; Gomes da Silva et al., 2011; AstudilloDefru et al., 2017b). With the increasing awareness of its importance for exoplanet study, characterization of high- energy radiation of a wide spectral range of stars is being advanced using data from HST (UV), ROSAT, XMMNewton, and GALEX (X-ray) and through the development of models to reconstruct the spectra in the wavelength range that is difficult to observe (e.g., Engle and Guinan, 2011; France et al., 2013, 2016; Stelzer et al., 2013; Loyd et al., 2016; Youngblood et al., 2016).

\subsection{Orbital architecture of the planetary system}

While we will likely discover tens to about one hundred Earth-sized planets around HZs in the coming decade, a substantially larger number of giant planets and/or planets in a variety of orbits that are easier to detect will also be discovered. For example, TESS will discover $>1000$ planets with radii larger than $2 R_{\oplus}$ (Sullivan et al., 2015). New ground-based transit surveys, including the Next Generation Transit Survey (Wheatley et al., 2013), will also contribute to unveiling the population of transiting planets. Continuous efforts in RV monitoring will also uncover more planets. In addition, using the astrometry method, the Gaia mission (Casertano et al., 2008) is estimated to discover $\sim 21,000$ large and distant planets during its nominal 5-year mission (Perryman et al., 2014). Large and distant planets will also continue to be targeted by ground-based direct-imaging observations, as well as by the future coronagraphic instruments on JWST, GMT, TMT, ELT, and WFIRST. These observations will add a significant number of samples to our catalogue of planetary systems whose major architectures (orbits, masses and/or radii of the planets) are known-How is such information related to the future biosignature search?

In general, such information potentially has indirect implications for planets of astrobiological interest through planet formation processes. For example, Earth-sized planets in the HZ of systems with a hot Jupiter (or hot Jupiters) may be volatile-rich in comparison to Earth, as a consequence of the migration process; specifically, hot Jupiters are believed to form farther out in the disk and then subsequently reach short orbital periods through viscous migration, during which process material from beyond the snow line is dragged inward, resulting in small planets with high volatile inventories (Raymond et al., 2006). Likewise, planets in systems whose architectures suggest inward migration (e.g., orbits in resonance) may have also formed beyond the snow line and be volatile-rich (Izidoro et al., 2014). However, planet formation and evolution processes include many uncertainties, and at this point we cannot make definitive predictions for the properties of individual Earth-sized planets, given the variety of compositions that can be produced under similar conditions (Carter-Bond et al., 2012). Conversely, once we have obtained the spectral information of the Earth-sized planets, this will provide, among other things, insights into the history of the system.

Another implication from orbital architectures is the effect of other planets in the same system on the long-term climate of the terrestrial planets of interest. Companion planets, particularly giants, will cause a terrestrial planet's orbit and obliquity to evolve (Berger, 1978; Laskar and Robutel, 1993), especially in the absence of strong tidal forces. Such long-term variations have been shown to be a powerful influence on climate, potentially inducing dramatic changes in global surface temperature, ice/snow cover, and 
possibly carbon cycling (Hays et al., 1976; Spiegel et al., 2010; Armstrong et al., 2014). Hence, climate modeling efforts that seek to place a potential biosignature into context should not neglect the orbital forcing of additional planets.

\subsection{Characterization of larger planets in the system}

While characterization of terrestrial planets will be exceedingly difficult, that of larger, gaseous planets will be more feasible during the coming era, providing us with a rich sample of characterized Jupiter-sized to Neptune-sized planets. For example, TESS and CHEOPS transit observations combined with RV measurement will give the accurate radius-mass relationship over a wide range of planetary sizes which will enable the determination of their gas fractions and infer possible formation scenarios. Atmospheric characterization of transiting gaseous planets will be performed with JWST, GMT, ELT, and TMT. Proposed missions, in particular FINESSE and ARIEL will carry out chemical surveys of 500 to 1000 transiting planets, preferentially targeting larger, warmer planets. Spectroscopic observations of phase variations could even provide threedimensional atmospheric mapping of gaseous planets, as demonstrated in Stevenson et al. (2014). Complementary to the transit observations, which are biased toward close-in planets, Jupiter-sized planets at distant orbits can be observed by direct imaging. Atmospheric characterization of young distant Jupiter-sized planets through direct-imaging observations has been successfully performed with existing $10 \mathrm{~m}$ class telescopes, and JWST, GMT, ELT, TMT, and WFIRST will also be able to perform high-contrast imaging of Jupiter-sized distant planets both in thermal light and in scattered light-If gas giants are present in the same planetary system as the terrestrial planets of astrobiological interest, what can characterization of these larger planets tell us about the habitability of terrestrial planetary companions?

The properties of gas giant atmospheres will provide additional insights into planet formation. For example, the core mass, which may be constrained from the mass-radius relationship, atmospheric properties, and/or the Love number (the value that describes the sensitivity of deformation of a body in response to a tidal force), could indicate the properties of the planet-forming region (e.g., Batygin et al., 2009; Nettelmann et al., 2010, 2011). If the atmospheric composition indicates a $\mathrm{C} / \mathrm{O}$ ratio significantly different from the host star's, it may have formed beyond the snow line (Öberg et al., 2011). Abundance ratios of certain elements may also reflect the composition of accreted planetesimals (Pinhas et al., 2016).

Due to the inherently complex nature of planet formation, however, at this point it is difficult to infer detailed characteristics of the disk from giant envelopes alone. Any further connection to the habitability of terrestrial planets will depend heavily on the robustness of formation and geophysical models (see, e.g., Lenardic and Crowley, 2012; Mordasini et al., 2012; Leconte et al., 2015; Stamenković and Seager, 2016). However, future development in planet formation and evolution theories may find more direct connections with habitability. Conversely, future characterization of terrestrial planets could provide insights into the formation and evolution pathways of the individual systems, giving useful constraints for the formation models.

\section{Prospects Beyond 2030}

In this section, we explore the possibilities beyond 2030 to further advance our investigations of potentially habitable planets. Section 6.1 is devoted to the introduction of the mission concepts currently being studied at NASA, and Section 6.2 includes other ideas that the community has been discussing for far-future projects.

\subsection{Mission concepts currently being studied in the United States}

Given the ongoing progress of exoplanet science and the further momentum for the field the near-future missions will provide, there is reason for optimism that an exoplanet mission capable of biosignature detection will be selected for development in the 2020s. In anticipation of the 2020 Decadal Survey, NASA is supporting two community-led mission studies that explore a range of science capabilities, costs, and mission architecture for direct imaging of habitable planets: HabEx and LUVOIR. HabEx would provide some general astrophysics capabilities, while the LUVOIR mission would give equal weight to exoplanet imaging and general astrophysics in its design. A third mission study, OST, is considering mid-IR detection of biosignatures in transit spectra post-JWST. All three studies are in the early stages of their mission concept definition, with many aspects still under discussion and subject to engineering trade studies. Final architecture reports will be delivered in early 2019.

6.1.1. Habitable Exoplanet Imaging Mission (HabEx). The HabEx study aims to take the first steps in the search for habitability and biosignatures. It is considering "smaller" telescopes with diameters in the $4-6.5 \mathrm{~m}$ size range, unobscured telescope apertures, and some combination of coronagraphs and starshade (or starshades). HabEx would be able to search the HZs of up to 40 nearby solar-type stars. For a conservative $\eta_{\oplus}$ of $10 \%$, HabEx would find a handful of terrestrial planets for spectroscopic follow-up at wavelengths mostly below $1 \mu \mathrm{m}$. Especially at the $4 \mathrm{~m}$ aperture size, spectroscopy of these at $\mathbb{R} \sim 140$ would require weeks of integration time per target, and rotational brightness modulation would be difficult to detect with fidelity. HabEx could provide a substantial science return for comparative planetology, with sub-Neptune-sized planets accessible around roughly a hundred to several hundred nearby stars and jovian planets around roughly a thousand. HabEx would carry one general astrophysics instrument, provisionally planned as a UV spectrograph. The HabEx engineering design work is led by NASA's Jet Propulsion Laboratory.

6.1.2. Large UltraViolet Optical and InfraRed surveyor (LUVOIR). The LUVOIR study has the greater ambition to survey a large sample of nearby star HZs in order to constrain the frequency of habitability and biosignatures. LUVOIR is baselining larger telescopes with diameters in the $9-15 \mathrm{~m}$ size range, providing access to $200-500 \mathrm{HZs}$ and a "conservative" yield of 20-50 terrestrial planets. Such large target samples are difficult to survey with starshades; thus, LUVOIR has baselined coronagraphs as its prime starlight suppression architecture. The constraints of existing launch vehicles require that the mission's large telescope aperture be realized with a segmented primary mirror, for 
FIG. 10. Solid lines: Thermal emission spectra of Earth, Venus, and Mars. The Earth spectrum is from Robinson et al. (2011). The spectra of Venus and Mars were modeled using the radiative transfer code SMART, assuming the 1D atmospheric profiles of each planet. Venus data is from Giada Arney, and Mars data is from Robinson and Crisp (2018). Dashed lines: Blackbody emission from a planet of the same radius with the approximately maximum brightness temperature of each planet in this range. See also Selsis et al. (2008) and Kaltenegger (2017).

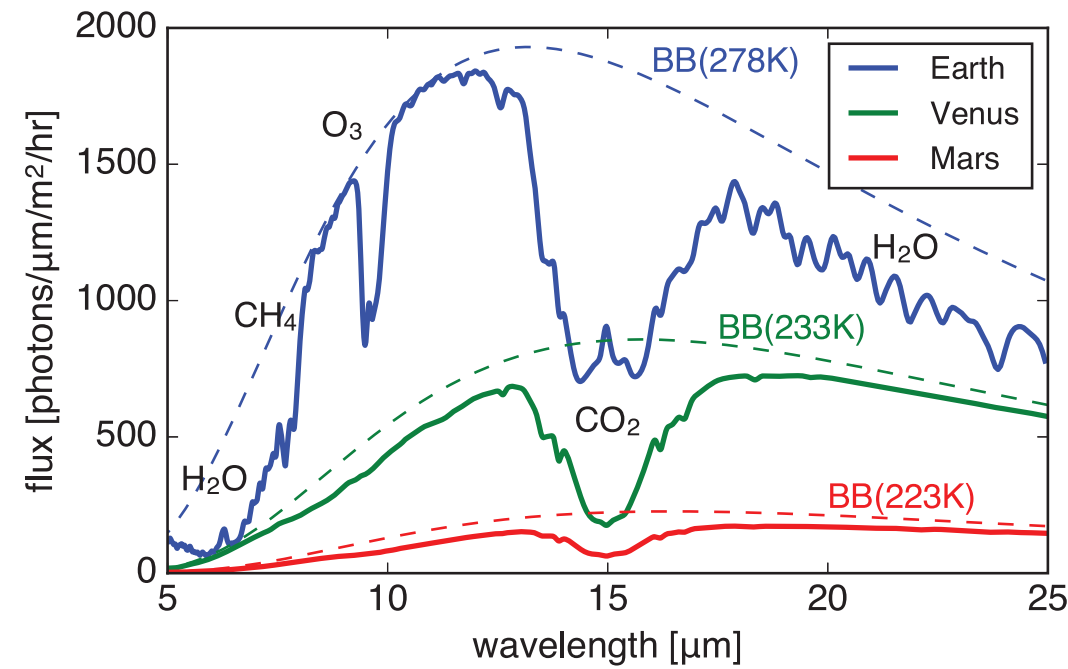

which coronagraphy is more technically challenging. LUVOIR's greater collecting area would allow spectra of $\mathrm{HZ}$ exoplanets to be made in roughly a day of integration time; the simulated observed data are shown by blue points in Figure 6 (Robinson et al., 2011); a subsample of at least a dozen targets could have spectra measured into the near-IR and have their rotational brightness modulation detected; the comparative planetology would be even richer than in the case of HabEx. Terrestrial planets at varying ages in varying orbits will provide insights into how planets have formed and how they evolve over geological time, including the "magma ocean" scenario (Hamano et al., 2015) and the coevolution of life and the planetary environment (e.g., Kaltenegger et al., 2008).

In addition to its coronagraph, LUVOIR would carry four other instruments for general astrophysics. LUVOIR is studying whether the general astrophysics camera might be calibrated well enough to provide sub-microarcsecond astrometry, which would enable detection of the stellar reflex motion of $\mathrm{HZ}$ terrestrial planets and thus the determination of their masses. The LUVOIR engineering design work is led by NASA's Goddard Space Flight Center.

6.1.3. Origins Space Telescope (OST). The OST study (Meixner et al., 2016) will characterize the atmospheres of nearby, transiting terrestrial exoplanets using the transmission and emission spectroscopy techniques. One of the primary goals of the OST mission is to search for and detect atmospheric biosignatures in multiple systems and assign probabilities to their origins. Like LUVOIR, OST would be a general astrophysics observatory but would operate at mid- and far-IR wavelengths $(6-600 \mu \mathrm{m})$. It is currently baselined to have a segmented primary mirror that is $9 \mathrm{~m}$ in diameter and utilizes an off-axis design. It would carry up to five instruments, one of which is being designed specifically to detect biosignatures in exoplanet atmospheres (Matsuo et al., 2016).

In the mid-IR, the main observable is a planet's dayside emission spectrum, as measured using the secondary eclipse technique. As discussed in Section 3.3, between 8 and $30 \mu \mathrm{m}$ where the SNR is favorable, there are prominent absorption features due to $\mathrm{CH}_{4}, \mathrm{CO}_{2}, \mathrm{O}_{3}, \mathrm{NH}_{3}, \mathrm{~N}_{2} \mathrm{O}$, and $\mathrm{SO}_{2}$, as well as the $\mathrm{H}_{2} \mathrm{O}$ vapor continuum. These features can distinguish a wet Earth-like planet from a dry, Venus-like planet with a dense $\mathrm{CO}_{2}$ atmosphere and a Mars-like planet with a thin $\mathrm{CO}_{2}$ atmosphere (Fig. 10). The strong $\mathrm{O}_{3}$ band at $9.7 \mu \mathrm{m}$ allows for the inference of $\mathrm{O}_{2}$, which is a powerful biosignature when combined with other out-of-equilibrium molecular species (such as $\mathrm{CH}_{4}$ at $7.7 \mu \mathrm{m}$ ). Additionally, emission spectroscopy uniquely probes a planet's thermal structure, which is critical toward assessing its habitability.

In addition to measuring planetary emission, mid-IR observations can take full advantage of a planet's transmission spectrum as measured during primary transit (Section 3.2). transmission observations place additional atmospheric constraints on the above-mentioned molecules at the planet's terminator. Furthermore, mid-IR transmission spectra are less affected by the high-altitude aerosols that tend to obscure spectral features at shorter wavelengths (e.g., $\mathrm{Hu}$ et al., 2013; Arney et al., 2016).

\subsection{Ideas for the far future}

Here we mention some of the more visionary ideas for the far-future missions found in the literature that could further advance our investigations of Earth-sized exoplanets in the search for life. The concepts introduced here are not around the corner in terms of technological development and funding, and these challenges will not be discussed in detail in this paper.

6.2.1. Direct imaging in the mid-IR. The idea of building a space-based interferometric direct-imaging observatory in the mid-IR for Earth-sized exoplanets was studied in proposed mission concepts such as the ESA-led Darwin (Léger et al., 1996; Fridlund, 2000) and the NASA-led TPF-I (Beichman et al., 1999; Lawson et al., 2007), but currently they are not actively studied. While a less challenging contrast of $10^{-7}$ is needed to study habitable exoplanets around solar-type stars in the mid-IR, going to wavelengths 10-15 times longer than the visible requires telescopes 10-15 times larger (Eq. 9). For the time being, the telescope dimensions required to study habitable exoplanets in the mid-IR $(>30 \mathrm{~m})$ can only be realized as interferometers: separate telescopes and a beam combiner distributed on multiple spacecrafts flying in formation. This complexity, combined with the mid-IR requirement to operate at cryogenic temperatures, led the US community to give first priority to architectures operating at visible wavelengths in 2011. Nevertheless, mid-IR high-contrast observations are 
desirable for characterizing thermal profiles and searching for certain atmospheric molecules of Earth-like planets around solar-type stars, which will be difficult with the near-future instruments. As discussed, signatures in the mid-IR range include various potential biosignatures (e.g., $\mathrm{O}_{3}, \mathrm{CH}_{4}$ ). Thermal emission spectra may also be used to estimate the planetary radius (Des Marais et al., 2002), which otherwise remains unobservable unless it transits. Furthermore, the time variation of thermal emission is affected by planetary obliquity (Gaidos and Williams, 2004; Gómez-Leal et al., 2012; Cowan et al., 2013) as well as thermal inertia (Cowan et al., 2012) and thus may be used to make inferences for these parameters.

6.2.2. ExoEarth Mapper. Ultimately, we would want a planet imager that has extremely high angular resolution, high enough to spatially resolve the exoplanetary surface (Enduring Quests, Daring Visions [NASA, December 2013], https://science.nasa.gov/astrophysics/documents). An interferometer in the visible that directly produces, for example, a $10 \times 10$ pixel map of the surface of an exoplanet, would provide critical, rich information regarding the planetary surface environment. The surface albedo heterogeneity would be directly observed, disclosing the distribution of patchy cloud cover, oceans, and continents. The spatial pattern of clouds would also reveal the atmospheric circulation and possibly imply the underlying topography (e.g., mountains). On compiling a time series, one could also measure the rotation rate and the obliquity of the planet, and the seasonality of surface features. We may even search for the spatial distributions of biological surface signatures such as vegetation's red edge and pigments, and their correlation with the distribution of habitats would enhance our confidence level that the detected signatures are indeed biotic. To spatially resolve the disk of an Earth analogue at $10 \mathrm{pc}$ distance, at the visible wavelengths, would require an interferometer with baselines of several hundred kilometers.

6.2.3. Telescope on the Moon. Like a free-flying space telescope, a telescope on the Moon (e.g., Burns and Mendell, 1988) shares the advantage of being outside Earth's atmosphere and having longer-duration access to its targets. The rigid ground of the lunar surface may make the construction of large telescopes and interferometers easier. However, this potential advantage is offset by the disadvantages of large day-night temperature swings, the $\sim 330 \mathrm{~h}$ lunar night, and contamination of optical elements by lunar dust. Still, the lunar far side remains the best site for a low-frequency radio telescope, as it is isolated from terrestrial radio interference.

6.2.4. One-hundred-meter-class ground-based telescope. The idea of $100 \mathrm{~m}$ class ground-based telescopes was once discussed at the European Southern Observatory (Dierickx and Gilmozzi, 2000). The concept for a $74 \mathrm{~m}$ telescope named the Colossus telescope has been proposed (Kuhn et al., 2014). It would likely be two or three decades before ground telescopes larger than ELTs will be pursued.

\section{Summary: Ideal Timeline}

This paper has explored the prospects of future observations to contribute to the general characterization of terres- trial planets in the HZs and to search for biosignatures. Here we summarize them in a serial timeline, for which different aspects are covered in Tables 1 and 2 .

Characterization of $\mathrm{HZ}$ terrestrial planets in the coming decade will feature transiting planets around late-type (M-type) stars. TESS will soon play the primary role in surveying transit signals of nearby short-orbit planets, including Earth-sized planets in HZs of late-type stars. CHEOPS will then provide measurements of radii down to sub-Neptune size with its ultrahigh photometric precision. These planetary systems around nearby late-type stars will allow for RV mass measurements by ground-based high-resolution spectrographs. The set of wellcharacterized planets in terms of radius and mass will advance the study of the mass-radius relationship of the close-in small planets. Meanwhile, the host stars and the planetary system architecture will be better characterized.

A small number of the discovered transiting potentially habitable planets around nearby late-type stars may be followed up by observations with JWST, if the noise floor is smaller than the expected atmospheric signals. An intensive use of the telescope for a few golden targets will assess whether such planets have atmospheres at all. If atmospheres and signs of habitability are found, a more thorough search for biosignatures may be conducted through transit spectroscopy with the intensive use of JWST or ground-based telescopes. The largest existing ground-based telescope might perhaps access the most nearby targets, upgrading high-resolution spectrographs and high-contrast imaging instruments.

ELTs (GMT, ELT, TMT) will start operation in the 2020s, and all of these telescopes are contemplating the instruments for characterization of template terrestrial planets using highresolution transmission spectroscopy, high-contrast imaging, and the high-resolution high-contrast method. Once such instruments are installed, they will offer invaluable opportunities to detect atmospheric signatures of $\mathrm{HZ}$ planets around late-type stars. This characterization of a handful of golden targets is a tremendous near-term opportunity to not just search for life but also test theories, in particular those about the loss and replenishment of atmospheres around terrestrial planets.

Investigation of potentially habitable planets around solartype (F-, G-, and K-type) stars will be facilitated from the mid2020s. Around the mid-2020s, HZ terrestrial transiting planets around these stars will be surveyed by PLATO. Together with mass measurements from the ground or the TTV method, the mass-radius relationship for these relatively distant planets will be derived; this will provide critical prior knowledge for future directly imaged planets. With luck, one or two targets may overlap with the nearby targets of future space-based directimaging missions.

WFIRST offers the first possibility to spectrally characterize these $\mathrm{HZ}$ planets around solar-type stars. If WFIRST is coupled with an external occulter (starshade) to sufficiently block the stellar light to the contrast level $10^{-10}$, it may be able to directly image Earth-sized planets in HZs of solar-type stars and take low-resolution spectra for crude characterization of their atmospheres. Without an external occulter, WFIRST will work with a coronagraph instrument that is expected to operate at contrast limits of about $2 \times 10^{-9}$ at 130 mas separations, enabling for the detection of larger planets, which potentially include terrestrial ones.

There are three options being considered for advancing our search for biosignatures, beyond the initial search that will be 
conducted over the next $\sim 10$ years. The first option is to investigate the same (and similar) targets as observed by JWST and/or ELTs (i.e., those around late-type stars) with the higher sensitivity (compared to JWST) and expanded wavelength coverage (compared to ELT's) provided by OST. The second option, HabEx, will target biosignatures on planets orbiting nearby solar-type stars with scattered light spectroscopy, while also enabling follow-up transit spectroscopy of the JWST/ ELTs targets in the UV to visible wavelengths. The third option, LUVOIR, would conduct a survey of biosignatures for planets around hundreds of stars via scattered light spectroscopy of those around solar-type stars as well as detailed follow-up of JWST/ELTs targets with transit spectroscopy in the UV to visible wavelengths. These options will allow our community to be responsive to the scientific and technological developments of the next few years. Which option we pursue will be decided by the next US Astrophysics Decadal Survey.

As argued throughout this paper and other papers in this series, finding inhabited planets will not end with the detection of a single feature of a biosignature candidate (or candidates). Falsepositive scenarios must be examined and ruled out based on the environmental context before claims of extraterrestrial life are made. Additional evidence implying habitable conditions will enhance our confidence level for the biological origin of the biosignature candidate. Ultimately, identifying inhabited planets will be the result of successive efforts that accumulate the evidence of the planetary environment, until one finds a set of signatures that cannot be explained by any known abiotic processes and can be reasonably explained by evoking the presence of a biosphere. Such efforts should rely on comprehensive characterization of individual planets and the planetary system properties provided by different observations surveyed in this paper, accompanied with the theoretical models of possible varieties in $\mathrm{HZ}$ terrestrial planets, both those with life and those without life.

In this paper, we also discussed the known difficulties in observationally obtaining some of the key parameters to evaluate habitability. Given the limited quality and quantity of the data available in the future, retrievals of the planetary properties will easily suffer from degeneracies that lead to inconclusive or biased interpretations. Thus, developing data analysis techniques and the framework to properly decode available data are essential. It is also important to further explore the methods of characterization, which would correspond to expanding Table 2 and filling in the blanks. Multiple novel ideas were presented in the past decade as reviewed in this paper, and more ideas may be expected to come.

The detection of life across light-year distances will perhaps be one of the most difficult measurements ever made, but powerful instruments and careful inquiry should indeed make it possible within the next few decades. No doubt the future will contain hurdles and discoveries that we cannot predict here. We hope, however, that this work will provide a guiding light to steer the way.

\section{Acknowledgments}

We would like to thank the NASA Astrobiology Program and the Nexus for Exoplanet System Science (NExSS) for their support for the NExSS Exoplanet Biosignatures Workshop. Conversations at this workshop, held in the summer of 2016, formed the basis for the drafting of the five review manuscripts in this issue. We also want to thank Mary Voytek, the senior scientist for Astrobiology, for her leadership of NExSS and her feedback on our organization of the workshop and papers. We also thank Natasha Batalha, Mercedes Lopez-Morales, John Fairweather, Avi M. Mandell, Vladimir Airapetian, Sarah Rugheimer, Antonio García Muñoz, and Simon Eriksson for the feedback during the community input period. We are also grateful to Motohide Tamura for the careful reading and feedback. We acknowledge Jason Tumlinson and Giada Arney for developing the LUVOIR tools used to create Figure 6. The comments from Ramses Ramirez and the other, anonymous reviewer greatly improved the clarity of the manuscript. Y.F. is grateful to David S. Amundsen, who gave insightful suggestions on the early draft, and to Teruyuki Hirano for helpful discussions. R.D. would like to thank Rory Barnes, Victoria Meadows, Rodrigo Luger, Jake Lustig-Yaeger, and Kimberly Bott for enlightening discussions. We thank Lucy Kwok for English proofreading of the manuscript. Y.F.'s research was supported by an appointment to the NASA Postdoctoral Program at the NASA Goddard Institute for Space Studies, administered by Universities Space Research Association under contract with NASA. D.A. acknowledges the support of the Center for Space and Habitability of the University of Bern and the National Centre for Competence in Research PlanetS supported by the Swiss National Science Foundation. R.D. is supported by the NASA Astrobiology Institute's Virtual Planetary Laboratory under Cooperative Agreement number NNA13AA93A. Part of the research was carried out at the Jet Propulsion Laboratory, California Institute of Technology, under a contract with NASA. Financial support for Open Access page charges was provided by the NASA Astrobiology Program, via the NASA Astrobiology Program, via the Nexus for Exoplanet Systems Science (NExSS).

\section{Author Disclosure Statement}

No competing financial interests exist.

\section{References}

Abe, Y., Abe-Ouchi, A., Sleep, N.H., and Zahnle, K.J. (2011) Habitable zone limits for dry planets. Astrobiology 11:443-460. Agol, E., Steffen, J., Sari, R., and Clarkson, W. (2005) On detecting terrestrial planets with timing of giant planet transits. Mon Not R Astron Soc 359:567-579.

Airapetian, V.S., Glocer, A., Gronoff, G., Hébrard, E., and Danchi, W. (2016) Prebiotic chemistry and atmospheric warming of early Earth by an active young Sun. Nat Geosci 9:452-455.

Airapetian, V.S., Glocer, A., Khazanov, G.V., Loyd, R.O.P., France, K., Sojka, J., Danchi, W.C., and Liemohn, M.W. (2017) "How Hospitable Are Space Weather Affected Habitable Zones? The Role of Ion Escape" The Astrophysical Journal Letters, Volume 836, Issue 1, article id. L3, 5 pp.

Angerhausen, D., Mandushev, G., Mandell, A., Dunham, E., Becklin, E., Collins, P., Hamilton, R., Logsdon, S.E., McElwain, M., McLean, I., Pfüller, E., Savage, M., Shenoy, S., Vacca, W., van Cleve, J., and Wolf, J. (2015) First exoplanet transit observation with the Stratospheric Observatory for Infrared Astronomy: confirmation of Rayleigh scattering in HD 189733 b with the High-Speed Imaging Photometer for Occultations. J Astron Telesc Instrum Syst 1:034002.

Anglada-Escudé, G., Arriagada, P., Vogt, S.S., Rivera, E.J., Butler, R.P., Crane, J.D., Shectman, S.A., Thompson, I.B., 
Minniti, D., Haghighipour, N., Carter, B.D., Tinney, C.G., Wittenmyer, R.A., Bailey, J.A., O'Toole, S.J., Jones, H.R.A., and Jenkins, J.S. (2012) A planetary system around the nearby M dwarf GJ 667C with at least one super-Earth in its habitable zone. Astrophys J 751:L16.

Anglada-Escudé, G., Amado, P.J., Barnes, J., Berdiñas, Z.M., Butler, R.P., Coleman, G.A., de la Cueva, I., Dreizler, S., Endl, M., Giesers, B. Jeffers, S.V., Jenkins, J.S., Jones, H.R., Kiraga, M., Kürster, M., López-González, M.J., Marvin, C.J., Morales., N., Morin, J., Nelson, R.P., Oritz, J.L., Ofir, A., Paardekooper, S.-J., Reiners, A., Rodríguez, E., RodríguezLópez, C., Sarmiento, L.F., Strachan, J.P., Tsapras, Y., Tuomi, M., and Zechmeister, M. (2016) A terrestrial planet candidate in a temperate orbit around Proxima Centauri. Nature 536:437-440.

Armstrong, J.C., Barnes, R., Domagal-Goldman, S., Breiner, J., Quinn, T.R., and Meadows, V.S. (2014) Effects of extreme obliquity variations on the habitability of exoplanets. Astrobiology 14:277-291.

Arney, G., Domagal-Goldman, S.D., Meadows, V.S., Wolf, E.T., Schwieterman, E., Charnay, B., Claire, M., Hébrard, E., and Trainer, M.G., (2016) The pale orange dot: the spectrum and habitability of hazy Archean Earth. Astrobiology 16:873-899.

Astudillo-Defru, N., Delfosse, X., Bonfils, C., Forveille, T., Lovis, C., and Rameau, J. (2017a) Magnetic activity in the HARPS Mdwarf sample. The rotation-activity relationship for very low-

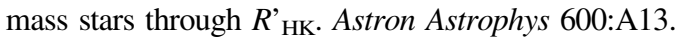

Astudillo-Defru, N., Forveille, T., Bonfils, X., Ségransan, D., Bouchy, F., Delfosse, X., Lovis, C., Mayor, M., Murgas, F., Pepe, F., Santos, N.C., Udry, S., and Wünsche, A. (2017b) The HARPS search for southern extra-solar planets XLI. A dozen planets around the M dwarfs GJ 3138, GJ 3323, GJ 273, GJ 628, and GJ 3293. arXiv:1703.05386. Available online at http://arxiv.org/abs/1703.05386

Bailey, J. (2007) Rainbows, polarization, and the search for habitable planets. Astrobiology 7:320-332.

Baldridge, A.M., Hook, S.J., Grove, C.I., and Rivera, G. (2009) The ASTER spectral library version 2.0. Remote Sens Environ 113:711-715.

Barnes, R. and Quinn, T. (2001) A statistical examination of the short-term stability of the $v$ Andromedae planetary system. Astrophys J 550:884-889.

Barnes, R., Mullins, K., Goldblatt, C., Meadows, V.S., Kasting, J.F., and Heller, R. (2013) Tidal Venuses: triggering a climate catastrophe via tidal heating. Astrobiology 13:225-250.

Barstow, J.K. and Irwin, P.G.J. (2016) Habitable worlds with JWST: transit spectroscopy of the TRAPPIST-1 system? Mon Not $R$ Astron Soc 461:L92-L96.

Barstow, J.K., Irwin, P.G.J., Kendrew, S., and Aigrain, S. (2016) Exoplanets with JWST: degeneracy, systematics and how to avoid them. Proc SPIE 9904, doi:10.1117/ 12.2232543 .

Batalha, N., Kalirai, J., Lunine, J., Clampin, M., and Lindler, D. (2015) Transiting Exoplanet Simulations with the James Webb Space Telescope. arXiv:1507.02655. Available online at http://arxiv.org/abs/1507.02655

Batygin, K., Bodenheimer, P., and Laughlin, G. (2009) Determination of the interior structure of transiting planets in multiple-planet systems. Astrophys J 704:L49-L53.

Beck, T., Broeg, C., Fortier, A., Cessa, V., Malvasio, L., Piazza, D., Benz, W., Thomas, N., Magrin, D., Viotto, V., Bergomi, M., Ragazzoni, R., Pagano, I., Peter, G., Buder, M., Plesseria, J.Y., Steller, M., Ottensamer, R., Ehrenreich, D., Van
Damme, C., Isaak, K., Ratti, F., Rando, N., and Ngan, I. (2016) CHEOPS: status summary of the instrument development. Proc SPIE 9904, doi:10.1117/12.2234562.

Beichman, C.A., Woolf, N.J., and Lindensmith, C.A. (1999) The Terrestrial Planet Finder (TPF): A NASA Origins Program to Search for Habitable Planets, JPL Publication 99-3, Jet Propulsion Laboratory, California Institute of Technology, Pasadena, CA.

Benneke, B. and Seager, S. (2012) Atmospheric retrieval for super-Earths: uniquely constraining the atmospheric composition with transmission spectroscopy. Astrophys $J$ 753, doi: 10.1088/0004-637X/753/2/100.

Berdyugina, S.V., Berdyugin, A.V., Fluri, D.M., and Piirola, V. (2008) First detection of polarized scattered light from an exoplanetary atmosphere. Astrophys J Lett 673, Issue 1, article id. L83.

Berdyugina, S.V., Berdyugin, A.V., Fluri, D.M., and Piirola, V. (2011) Polarized reflected light from the exoplanet HD 189733b: first multicolor observations and confirmation of detection. Astrophys $J$ Lett 728, Issue 1, article id. L6.

Berger, A.L. (1978) Long-term variations of daily insolation and quaternary climatic changes. Journal of the Atmospheric Sciences 35:2362-2367.

Berta-Thompson, Z.K., Irwin, J., Charbonneau, D., Newton, E.R., Dittmann, J.A., Astudillo-Defru, N., Bonfils, X., Gillon, M., Jehin, E., Stark, A.A., Stalder, B., Bouchy, F., Delfosse, X., Forveille, T., Lovis, C., Mayor, M., Neves, V., Pepe, F., Santos, N.C., Udry, S., and Wünsche, A. (2015) A rocky planet transiting a nearby low-mass star. Nature 527:204-207.

Bétrémieux, Y. and Kaltenegger, L. (2013) Transmission spectrum of Earth as a transiting exoplanet from the ultraviolet to the near-infrared. Astrophys J 772:L31.

Bétrémieux, Y. and Kaltenegger, L. (2014) Impact of atmospheric refraction: how deeply can we probe exo-Earth's atmospheres during primary eclipse observations? Astrophys $J$ 791, doi:10.1088/0004-637X/791/1/7.

Birkby, J.L., de Kok, R.J., Brogi, M., de Mooij, E.J.W., Schwarz, H., Albrecht, S., and Snellen, I.A.G. (2013) Detection of water absorption in the day side atmosphere of HD $189733 \mathrm{~b}$ using ground-based high-resolution spectroscopy at $3.2 \mu \mathrm{m}$. Mon Not $R$ Astron Soc 436:L35-L39.

Boisse, I., Bonfils, X., and Santos, N.C. (2012) SOAP. A tool for the fast computation of photometry and radial velocity induced by stellar spots. Astron Astrophys 545:A109.

Bonfils, X., Delfosse, X., Udry, S., Forveille, T., Mayor, M., Perrier, C., Bouchy, F., Gillon, M., Lovis, C., Pepe, F., Queloz, D., Santos, N.C., Ségransan, D., and Bertaux, J.-L. (2013) The HARPS search for southern extra-solar planets XXXI. The M-dwarf sample. Astron Astrophys 549:A109.

Borucki, W.J., Koch, D.G., Basri, G., Batalha, N., Boss, A., Brown, T.M., Caldwell, D., Christensen-Dalsgaard, J., Cochran, W.D., DeVore, E., Dunham, E.W., Dupree, A.K., Gautier, T.N., III, Geary, J.C., Gilliland, R., Gould, A., Howell, S.B., Jenkins, J.M., Kjeldsen, H., Latham, D.W., Lissauer, J.J., Marcy, G.W., Monet, D.G., Sasselov, D., Tarter, J., Charbonneau, D., Doyle, L., Ford, E.B., Fortney, J., Holman, M.J., Seager, S., Steffen, J.H., Welsh, W.F., Allen, C., Bryson, S.T., Buchhave, L., Chandrasekaran, H., Christiansen, J.L., Ciardi, D., Clarke, B.D., Dotson, J.L., Endl, M., Fischer, D., Fressin, F., Haas, M., Horch, E., Howard, A., Isaacson, H., Kolodziejczak, J., Li, J., MacQueen, P., Meibom, S., Prsa, A., Quintana, E.V., Rowe, J., Sherry, W., Tenenbaum, P., Torres, G., Twicken, J.D., Van Cleve, J., Walkowicz, L., and Wu, H. (2011) Characteristics of Kepler 
planetary candidates based on the first data set: the majority are found to be Neptune-size and smaller. Astrophys $J$ 728, doi:10.1088/0004-637X/728/2/117.

Bott, K., Bailey, J., Kedziora-Chudczer, L., Cotton, D.V., Lucas, P.W., Marshall, J.P., and Hough, J.H. (2016) The polarization of HD 189733. Monthly Notices of the Royal Astronomical Society: Letters 459:L109-L113.

Bourrier, V., Lecavelier des Etangs, A., Ehrenreich, D., Tanaka, Y.A., and Vidotto, A.A. (2016) An evaporating planet in the wind: stellar wind interactions with the radiatively braked exosphere of GJ 436 b. Astron Astrophys 591:A121.

Boutle, I.A., Mayne, N.J., Drummond, B., Manners, J., Goyal, J., Hugo Lambert, F., Acreman, D.M., and Earnshaw, P.D. (2017) Exploring the climate of Proxima B with the Met Office Unified Model. Astron Astrophys 601, id. A120, 13 pp.

Brandt, T.D. and Spiegel, D.S. (2014) Prospects for detecting oxygen, water, and chlorophyll on an exo-Earth. Proc Natl Acad Sci USA 111:13278-13283.

Brion, J., Chakir, A., Charbonnier, J., Daumont, D., Parisse, C., and Malicet, J. (1998) Absorption Spectra measurements for the ozone molecule in the $350-830 \mathrm{~nm}$ region. J Atmos Chem 30:291-299.

Brogi, M., Snellen, I.A.G., de Kok, R.J., Albrecht, S., Birkby, J., and de Mooij, E.J.W. (2012) The signature of orbital motion from the dayside of the planet $\tau$ Boötis b. Nature 486:502-504.

Burke, B.F. (1992) Searching for exoplanets. In Targets for Space-Based Interferometry, ESA SP 354, European Space Agency, Paris, pp 81-83 (SEE N93-31750 12-89).

Burke, C.J., Christiansen, J.L., Mullally, F., Seader, S., Huber, D., Rowe, J.F., Coughlin, J.L., Thompson, S.E., Catanzarite, J., Clarke, B.D., Morton, T.D., Caldwell, D.A., Bryson, S.T., Haas, M.R., Batalha, N.M., Jenkins, J.M., Tenenbaum, P., Twicken, J.D., Li, J., Quintana, E., Barclay, T., Henze, C.E., Borucki, W.J., Howell, S.B., and Still, M. (2015) Terrestrial planet occurrence rates for the Kepler GK dwarf sample. Astrophys $J$ 809, doi:10.1088/0004-637X/809/1/8.

Burns, J.O. and Mendell, W.W. (1988) Future Astronomical Observatories on the Moon, NASA Conference Publication 2489, NASA, Washington, DC.

Carter-Bond, J.C., O'Brien, D.P., and Raymond, S.N. (2012) The compositional diversity of extrasolar terrestrial planets. II. Migration simulations. Astrophys J 760, doi:10.1088/ 0004-637X/760/1/44.

Casertano, S., Lattanzi, M.G., Sozzetti, A., Spagna, A., Jancart, S., Morbidelli, R., Pannunzio, R., Pourbaix, D., and Queloz, D. (2008) Double-blind test program for astrometric planet detection with Gaia. Astron Astrophys 482:699-729.

Cash, W. (2006) Detection of Earth-like planets around nearby stars using a petal-shaped occulter. Nature 442:51-53.

Cassan, A., Kubas, D., Beaulieu, J.-P., Dominik, M., Horne, K., Greenhill, J., Wambsganss, J., Menzies, J., Williams, A., Jørgensen, U.G., Udalski, A., Bennett, D.P., Albrow, M.D., Batista, V., Brillant, S., Caldwell, J.A.R., Cole, A., Coutures, Ch., Cook, K.H., Dieters, S., Dominis Prester, D., Donatowicz, J., Fouqué, P., Hill, K., Kains, N., Kane, S., Marquette, J.-B., Martin, R., Pollard, K.R., Sahu, K.C., Vinter, C., Warren, D., Watson, B., Zub, M., Sumi, T., Szymański, M.K., Kubiak, M., Poleski, R., Soszynski, I., Ulaczyk, K., Pietrzyński, G., and Wyrzykowski, Ł. (2012) One or more bound planets per Milky Way star from microlensing observations. Nature 481:167-169.

Catanzarite, J. and Shao, M. (2011) The occurrence rate of Earth analog planets orbiting Sun-like stars. Astrophys $J$ 738, doi:10.1088/0004-637X/738/2/151.
Catling, D.C., Krissansen-Totton, J., Kiang, N.Y., Crisp, D., Robinson, T.D., DasSarma, S., Rushby, A.J., Del Genio, A., Bains, W., and Domagal-Goldman, S. (2018) Exoplanet biosignatures: a framework for their assessment. Astrobiology 18:709-738.

Charbonneau, D., Brown, T.M., Noyes, R.W., and Gilliland, R.L. (2002) Detection of an extrasolar planet atmosphere. Astrophys J 568, doi:10.1086/338770.

Charbonneau, D., Berta, Z.K., Irwin, J., Burke, C.J., Nutzman, P., Buchhave, L.A., Lovis, C., Bonfils, X., Latham, D.W., Udry, S., Murray-Clay, R.A., Holman, M.J., Falco, E.E., Winn, J.N., Queloz, D., Pepe, F., Mayor, M., Delfosse, X., and Forveille, T. (2009) A super-Earth transiting a nearby low-mass star. Nature 462:891-894.

Checlair, J., McKay, C.P., and Imanaka, H. (2016) Titan-like exoplanets: variations in geometric albedo and effective transit height with haze production rate. Planet Space Sci 129:1-12.

Cheng, B., Lu, H., Chen, H., Bahou, M., Lee, Y., Mebel, A.M., Lee, L.C., Liang, M., and Yung, Y.L. (2006) Absorption cross sections of $\mathrm{NH}_{3}, \mathrm{NH}_{2} \mathrm{D}, \mathrm{NHD}_{2}$, and $\mathrm{ND}_{3}$ in the Spectral Range 140-220 nm and implications for planetary Isotopic fractionation. Astrophys $J$ 647:1535-1542.

Coquart, B., Jenouvrier, A., and Merienne, M.F. (1995) The $\mathrm{NO}_{2}$ absorption spectrum. II. Absorption cross-sections at low temperatures in the $400-500 \mathrm{~nm}$ region. J Atmos Chem 21:251-261.

Cowan, N.B. and Fujii, Y. (2017) Mapping exoplanets. arXiv: 1704.07832. Available online at http://arxiv.org/abs/1704 .07832

Cowan, N.B. and Strait, T.E. (2013) Determining reflectance spectra of surfaces and clouds on exoplanets. Astrophys $J$ 765:L17.

Cowan, N.B., Algol, E., Meadows, V.S., Robinson, T., Livengood, T.A., Deming, D., Lisse, C.M., A'Hearn, M.F., Wellnitz, D.D., Seager, S., and Charbonneau, D. (2009) Alien maps of an ocean-bearing world. Astrophys J 700:915-923.

Cowan, N.B., Robinson, T., Livengood, T.A., Deming, D., Agol, E., A'Hearn, M.F., Charbonneau, D., Lisse, C.M., Meadows, V.S., Seager, S., Shields, A.L., and Wellnitz, D.D. (2011) Rotational variability of Earth's polar regions: implications for detecting snowball planets. Astrophys $J$ 731, doi: 10.1088/0004-637X/731/1/76.

Cowan, N.B., Voigt, A., and Abbot, D.S. (2012) Thermal phases of Earth-like planets: estimating thermal inertia from eccentricity, obliquity, and diurnal forcing. Astrophys $J$ 757, Issue 1, article id. 80, 13 pp.

Cowan, N.B., Fuentes, P.A., and Haggard, H.M. (2013) Light curves of stars and exoplanets: estimating inclination, obliquity and albedo. Monthly Notices of the Royal Astronomical Society 434:2465-2479.

Cowan, N.B., Greene, T., Angerhausen, D., Batalha, N.E., Clampin, M., Colon, K., Crossfield, I.J.M., Fortney, J.J., Gaudi, B.S., Harrington, J., Iro, N., Lillie, C.F., Linsky, J.L., López-Morales, M., Mandel, A.M., and Stevenson, K.B., on behalf of Exo PAG SAG-10. (2015) Characterizing transiting planet atmospheres through 2025. Publ Astron Soc Pac 127: 311-327.

Crepp, J.R., Crass, J., Bechter, A., Crass, J., King, D., Bechter, A., Bechter, E., Ketterer, R., Reynolds, R., Hinz, P., Kopon, D., Cavalieri, D., Fantano, L., Koca, C., Onuma, E., Stapelfeldt, K., Thomes, J., Wall, S., Macenka, S., McGuire, J., Korniski, R., Zugby, L., Eisner, J., Gaudi, B.S., Hearty, F., Kratter, K., Kuchner, M., Micela, G., Nelson, M., Pagano, I., 
Quirrenbach, A., Schwab, C., Skrutskie, M., Sozzetti, A., Woodward, C., and Zhao, B. (2016) iLocater: a diffractionlimited Doppler spectrometer for the Large Binocular Telescope. Proc SPIE 9908, doi:10.1117/12.2233135.

de Kok, R.J., Brogi, M., Snellen, I.A.G., Birkby, J., Albrecht, S., and de Mooij, E.J.W. (2013) Detection of carbon monoxide in the high-resolution day-side spectrum of the exoplanet HD 189733b. Astron Astrophys 554:A82.

Delfosse, X., Donati, J.-F., Kouach, D., Hébrard, G., Doyon, R., Artigau, E., Bouchy, F., Boisse, I., Brun, A.S., Hennebelle, P., Widemann, T., Bouvier, J., Bonfils, X., Morin, J., Moutou, C., Pepe, F., Udry, S., do Nascimento, J.D., Alencar, S.H.P., Castilho, B.V., Martioli, E., Wang, S.Y., Figueira, P., Santos, N.C., and the SPIRou Science Team. (2013) World-leading science with SPIRou-the nIR spectropolarimeter/highprecision velocimeter for CFHT. Proceedings of the Annual meeting of the French Society of Astronomy and Astrophysics, Société Francaise d'Astronomie et d'Astrophysique (S2FA), pp 497-508.

Deming, L.D. and Seager, S. (2017) Illusion and reality in the atmospheres of exoplanets. J Geophys Res: Planets 122:53-75.

Deming, D. and Sheppard, K. (2017) Spectral resolution-linked bias in transit spectroscopy of extrasolar planets. arXiv: 1705.00625. Available online at http://arxiv.org/abs/1705 .00625

Deming, D., Seager, S., Winn, J., Miller-Ricci, E., Clampin, M., Lindler, D., Greene, T., Charbonneau, D., Laughlin, G., Ricker, G., Lathan, D., and Ennico, K. (2009) Discovery and characterization of transiting super Earths Using an all-sky transit survey and follow-up by the James Webb Space Telescope. Publ Astron Soc Pac 121, doi:10.1086/605913.

Demory, B.-O., de Wit, J., Lewis, N., Fortney, J., Zsom, A., Seager, S., Knutson, H., Heng, K., Madhusudhan, N., Gillon, M., Barclay, T., Desert, J.-M., Parmentier, V., and Cowan, N.B. (2013) Inference of inhomogeneous clouds in an exoplanet atmosphere. Astrophys $J$ 776:L25.

Demory, B.-O., Gillon, M., de Wit, J., Madhusudhan, N., Bolmont, E., Heng, K., Kataria, T., Lewis, N., Hu, R., Krick, J., Stamenković, V., Benneke, B., Kane, S., and Queloz, D. (2016) A map of the large day-night temperature gradient of a super-Earth exoplanet. Nature 532:207-209.

Des Marais, D.J., Harwit, M.O., Jucks, K.W., Kasting, J.F., Lin, D.N., Lunine, J.I., Schneider, J., Seager, S., Traub, W.A., and Woolf, N.J. (2002) Remote sensing of planetary properties and biosignatures on extrasolar terrestrial planets. Astrobiology 2:153-181.

de Wit, J., Gillon, M., Demory, B.-O., and Seager, S. (2012) Towards consistent mapping of distant worlds: secondaryeclipse scanning of the exoplanet HD 189733b. Astron Astrophys 548:A128.

Dierickx, P. and Gilmozzi, R. (2000) Progress of the OWL 100m telescope conceptual design. Proc SPIE 4004, doi:10.1117/ 12.393934.

Dittmann, J.A., Irwin, J.M., Charbonneau, D., Bonfils, X., Astudillo-Defru, N., Haywood, R.D., Berta-Thompson, Z.K., Newton, E.R., Rodriguez, J.E., Winters, J.G., Tan, T.-G., Almenara, J.-M., Bouchy, F., Delfosse, X., Forveille, T., Lovis, C., Murgas, F., Pepe, F., Santos, N.C., Udry, S., Wünsche, A., Esquerdo, G.A., Latham, D.W., and Dressing, C.D. (2017) A temperate rocky super-Earth transiting a nearby cool star. Nature 544:333-336.

Domagal-Goldman, S.D., Meadows, V.S., Claire, M.W., and Kasting, J.F. (2011) Using biogenic sulfur gases as remotely detectable biosignatures on anoxic planets. Astrobiology 11: 419-441.
Domagal-Goldman, S.D., Segura, A., Claire, M.W., Robinson, T.D., and Meadows, V.S. (2014) Abiotic ozone and oxygen in atmospheres similar to prebiotic Earth. Astrophys J 792, doi: 10.1088/0004-637X/792/2/90.

Dorn, C., Venturini, J., Khan, A., Heng, K., Alibert, Y., Helled, R., Rivoldini, A., and Benz, W. (2017a) A generalized Bayesian inference method for constraining the interiors of super Earths and sub-Neptunes. Astron Astrophys 597:A37.

Dorn, C., Hinkel, N.R., and Venturini, J. (2017b) Bayesian analysis of interiors of HD 219134b, Kepler-10b, Kepler-93b, CoRoT-7b, 55 Cnc e, and HD 97658b using stellar abundance proxies. Astron Astrophys 597:A38.

Dorn, R.J., Follert, R., Bristow, P., Cumani, C., Eschbaumer, S., Grunhut, J., Haimerl, A., Hatzes, A., Heiter, U., Hinterschuster, R., Ives, D.J., Jung, Y., Kerber, F., Klein, B., Lavaila, A., Lizon, J.L., Löwinger, T., Molina-Conde, I., Nicholson, B., Marquart, T., Oliva, E., Origlia, L., Pasquini, L., Paufique, J., Piskunov, N., Reiners, A., Seemann, U., Stegmeier, J., Stempels, E., and Tordo, S. (2016) The " +" for CRIRES: enabling better science at infrared wavelength and high spectral resolution at the ESO VLT. Proc SPIE 9908, doi:10.1117/12.2232837.

Doyle, L.R., Carter, J.A., Fabrycky, D.C., Slawson, R.W., Howell, S.B., Winn, J.N., Orosz, J.A., Prša, A., Welsh, W.F., Quinn, S.N., Latham, D., Torres, G., Buchhave, L.A., Marcy, G.W., Fortney, J.J., Shporer, A., Ford, E.B., Lissauer, J.J., Ragozzine, D., Rucker, M., Batalha, N., Jenkins, J.M., Borucki, W.J., Koch, D., Middour, C.K., Hall, J.R., McCauliff, S., Fanelli, M.N., Quintana, E.V., Holman, M.J., Caldwell, D.A., Still, M., Stefanik, R.P., Brown, W.R., Esquerdo, G.A., Tang, S., Furesz, G., Geary, J.C., Berlind, P., Calkins, M.L., Short, D.R., Steffen, J.H., Sasselov, D., Dunham, E.W., Cochran, W.D., Boss, A., Haas, M.R., Buzasi, D., and Fischer, D. (2011) Kepler-16: a transiting circumbinary planet. Science 333:1602-1606.

Dressing, C.D. and Charbonneau, D. (2013) The occurrence rate of small planets around small stars. Astrophys $J$ 767, doi: 10.1088/0004-637X/767/1/95.

Dressing, C.D. and Charbonneau, D. (2015) The occurrence of potentially habitable planets orbiting $M$ dwarfs estimated from the full Kepler dataset and an empirical measurement of the detection sensitivity. Astrophys $J$ 807, doi:10.1088/0004$637 X / 807 / 1 / 45$.

Dumusque, X., Udry, S., Lovis, C., Santos, N.C., and Monteiro, M.J.P.F.G. (2011a) Planetary detection limits taking into account stellar noise. I. Observational strategies to reduce stellar oscillation and granulation effects. Astron Astrophys 525:A140.

Dumusque, X., Santos, N.C., Udry, S., Lovis, C., and Bonfils, X. (2011b) Planetary detection limits taking into account stellar noise. II. Effect of stellar spot groups on radialvelocities. Astron Astrophys 527:A82.

Ehrenreich, D., Bourrier, V., Wheatley, P.J., Lecavelier des Etangs, A., Hébrard, G., Udry, S., Bonfils, X., Delfosse, X., Désert, J.-M., Sing, D.K., and Vidal-Madjar, A. (2015) A giant comet-like cloud of hydrogen escaping the warm Neptune-mass exoplanet GJ 436b. Nature 522:459-461.

Elachi, C., Angel, R., Beichman, C.A., Boss, A., Brown, R., Dressler, A., Dyson, F., Fanson, J., Ftaclas, C., Goad, L., Klein, M., Leger, A., Lillie, C., Peale, S., Peterson, D., Reasenberg, B., Sandler, D., Shao, M., Simon, R., and Tenerelli, D. (1996) A Road Map for the Exploration of Neighboring Planetary Systems (ExNPS), Technical Report, Jet Propulsion Laboratory, California Institute of Technology, Pasadena, CA. 
Engle, S.G. and Guinan, E.F. (2011) Red dwarf stars: ages, rotation, magnetic dynamo activity and the habitability of hosted planets. In $9^{\text {th }}$ Pacific Rim Conference on Stellar Astrophysics, ASP Conference Series Vol. 451, edited by S. Qain, K. Leung, L. Zhu, and S. Kwok, Astronomical Society of the Pacific, San Francisco, CA, pp 285-294.

Feng, Y.K., Robinson, T.D., Fortney, J.J., Lupu, R.E., Marley, M.S., Lewis, N.K., Macintosh, B., and Line, M.R. (2018) Characterizing Earth analogs in reflected light: atmospheric retrieval studies for future space telescopes. Astron $J$ 155, Issue 5, article id. 200.

Fischer, D.A., Anglada-Escude, G., Arriagada, P., Baluev, R.V., Bean, J.L., Bouchy, F., Buchhave, L.A., Carroll, T., Chakraborty, A., Crepp, J.R., Dawson, R.I., Diddams, S.A., Dumusque, X., Eastman, J.D., Endl, M., Figueira, P., Ford, E.B., Foreman-Mackey, D., Fournier, P., Fürész, G., Gaudi, B.S., Gregory, P.C., Grundahl, F., Hatzes, A.P., Hébrard, G., Herrero, E., Hogg, D.W., Howard, A.W., Johnson, J.A., Jorden, P., Jurgenson, C.A., Latham, D.W., Laughlin, G., Loredo, T.J., Lovis, C., Mahadevan, S., McCracken, T.M., Pepe, F., Perez, M., Phillips, D.F., Plavchan, P.P., Prato, L., Quirrenbach, A., Reiners, A., Robertson, P., Santos, N.C., Sawyer, D., Segransan, D., Sozzetti, A., Steinmetz, T., Szentgyorgyi, A., Udry, S., Valenti, J.A., Wang, S.X., Wittenmyer, R.A., and Wright, J.T. (2016) State of the field: extreme precision radial velocities. Publ Astron Soc Pac 128, doi:10.1088/1538-3873/128/964/066001.

Follert, R., Dorn, R.J., Oliva, E., Lizon, J.L., Hatzes, A., Piskunov, N., Reiners, A., Seemann, U., Stempels, E., Heiter, U., Marquart, T., Lockhart, M., Anglada-Escude, G., Löwinger, T., Baade, D., Grunhut, J., Bristow, P., Klein, B., Jung, Y., Ives, D.J., Kerber, F., Pozna, E., Paufique, J., Kaeufl, H.U., Origlia, L., Valenti, E., Gojak, D., Hilker, M., Pasquini, L., Smette, A., and Smoker, J. (2014) CRIRES+: a crossdispersed high-resolution infrared spectrograph for the ESO VLT. Proc SPIE 9147, doi:10.1117/12.2054197.

Ford, E.B., Seager, S., and Turner, E.L. (2001) Characterization of extrasolar terrestrial planets from diurnal photometric variability. Nature 412:885-887.

Fortney, J.J., Marley, M.S., and Barnes, J.W. (2007) Planetary radii across five orders of magnitude in mass and stellar insolation: application to transits. Astrophys J 659:1661-1672.

France, K., Froning, C.S., Linsky, J.L., Roberge, A., Stocke, J.T., Tian, F., Bushinsky, R., Désert, J.-M., Mauas, P., Vieytes, M., and Walkowicz, L. (2013) The ultraviolet radiation environment around $\mathrm{M}$ dwarf exoplanet host stars. Astrophys $J$ 763, doi:10.1088/0004-637X/763/2/149.

France, K., Parke Loyd, R.O., Youngblood, A., Brown, A., Schneider, P.C., Hawley, S.L., Froning, C.S., Linsky, J.L., Roberge, A., Buccino, A.P., Daveneport, J.R.A., Fontenla, J.M., Kaltenegger, L., Kowalski, A.F., Mauas, P.J.D., Miguel, Y., Redfield, S., Rugheimer, S., Tian, F., Vieytes, M.C., Walkowicz, L.M., and Weisenburger, K.L. (2016) The MUSCLES treasury survey. I. Motivation and overview. Astrophys J 820, doi:10.3847/0004-637X/820/2/89.

Fridlund, C.V.M. (2000) Darwin - The Infrared Space Interferometry Mission. ESA Bulletin 103:20-25.

Fujii, Y. and Kawahara, H. (2012) Mapping Earth analogs from photometric variability: spin-orbit tomography for planets in inclined orbits. Astrophys J 755, doi:10.1088/0004-637X/755/2/101.

Fujii, Y., Kawahara, H., Suto, Y., Taruya, A., Fukuda, S., Nakajima, T., and Turner, E.L. (2010) Colors of a second Earth: estimating the fractional areas of ocean, land, and vegetation of Earth-like exoplanets. Astrophys J 715, doi:10.1088/0004-637X/715/2/866.
Fujii, Y., Kawahara, H., Suto, Y., Fukuda, S., Nakajima, T., Livengood, T.A., and Turner, E.L. (2011) Colors of a second Earth. II. Effects of clouds on photometric characterization of Earth-like exoplanets. Astrophys J 738, doi:10.1088/0004637X/738/2/184.

Fujii, Y., Turner, E.L., and Suto, Y. (2013) Variability of water and oxygen absorption bands in the disk-integrated spectra of Earth. Astrophys $J$ 765, doi:10.1088/0004-637X/765/2/76.

Fujii, Y., Kimura, J., Dohm, J., and Ohtake, M. (2014) Geology and photometric variation of Solar System bodies with minor atmospheres: implications for solid exoplanets. Astrobiology 14:753-768.

Fujii, Y., Del Genio, A.D., and Amundsen, D.S. (2017a) NIRdriven moist upper atmospheres of synchronously rotating temperate terrestrial exoplanets. Astrophys $J$ 848, doi: 10.3847/1538-4357/aa8955.

Fujii, Y., Lustig-Yaeger, J., and Cowan, N.B. (2017b) Rotational spectral unmixing of exoplanets: degeneracies between surface colors and geography. Astron J 154, doi:10.3847/ 1538-3881/aa89f1.

Fulton, B.J., Petigura, E.A., Howard, A.W., Isaacson, H., Marcy, G.W., Cargile, P.A., Hebb, L., Weiss, L.M., Johnson, J.A., Morton, T.D., Sinukoff, E., Crossfield, I.J.M., and Hirsch, L.A. (2017) The California-Kepler survey. III. A gap in the radius distribution of small planets. arXiv:1703.10375. Available online at http://arxiv.org/abs/1703.10375

Gaidos, E. (2013) Candidate planets in the habitable zones of Kepler stars. Astrophys J 770, doi:10.1088/0004-637X/770/ 2/90.

Gaidos, E. and Williams, D.M. (2004) Seasonality on terrestrial extrasolar planets: inferring obliquity and surface conditions from infrared light curves. New Astronomy 10:67-77.

García Muñoz, A. and Mills, F.P. (2012) The June 2012 transit of Venus. Framework for interpretation of observations. Astron Astrophys 547:A22.

García Muñoz, A. and Pallé, E. (2011) Lunar eclipse theory revisited: scattered sunlight in both the quiescent and the volcanically perturbed atmosphere. J Quant Spectrosc Radiat Transf 112:1609-1621.

García Muñoz, A., Zapatero Osorio, M.R., Barrena, R., MontañésRodríguez, P., Martín, E.L., and Pallé, E. (2012) Glancing views of the Earth: from a lunar eclipse to an exoplanetary transit. Astrophys J 755, doi:10.1088/0004-637X/755/2/103.

Gillon, M., Jehin, E., Lederer, S.M., Delrez, L., de Wit, J., Burdanov, A., Van Grootel, V., Burgasser, A.J., Triaud, A.H.M.J., Opitom, C., Demory, B.-O., Sahu, D.K., Bardalez Gagliuffi, D., Magain, P., and Queloz, D. (2016) Temperate Earth-sized planets transiting a nearby ultracool dwarf star. Nature 533:221-224.

Gillon, M., Triaud, A.H., Demory, B.O., Jehin, E., Agol, E., Deck, K.M., Lederer, S.M., de Wit, J., Burdanov, A., Ingalls, J.G., Bolmont, E., Leconte, J., Raymond, S.N., Selsis, F., Turbet, M., Barkaoui, K., Burgasser, A., Burleigh, M.R., Carey, S.J., Chaushev, A., Copperwheat, C.M., Delrez, L., Fernandes, C.S., Holdsworth, D.L., Kotze, E.J., Van Grootel, V., Almleaky, Y., Benkhaldoun, Z., Magain, P., and Queloz, D. (2017) Seven temperate terrestrial planets around the nearby ultracool dwarf star TRAPPIST-1. Nature 542:456460.

Gomes da Silva, J., Santos, N.C., Bonfils, X., Delfosse, X., Forveille, T., and Udry, S. (2011) Long-term magnetic activity of a sample of M-dwarf stars from the HARPS program. I. Comparison of activity indices. Astron Astrophys 534, id. A30, 17 pp. 
Gómez-Leal, I., Pallé, E., and Selsis, F. (2012) Photometric variability of the disk-integrated thermal emission of the Earth. Astrophys J 752, doi:10.1088/0004-637X/752/1/28.

Greene, T.P., Line, M.R., Montero, C., Fortney, J.J., LustigYaeger, J., and Luther, K. (2016) Characterizing transiting exoplanet atmospheres with JWST. Astrophys J 817, doi: 10.3847/0004-637X/817/1/17.

Grenfell, J.L., Grießmeier, J.M., von Paris, P., Patzer, A.B.C., Lammer, H., Stracke, B., Gebauer, S., Schreier, F., and Rauer, H. (2012) Response of atmospheric biomarkers to NOx-induced photochemistry generated by stellar cosmic rays for Earth-like planets in the habitable zone of $\mathrm{M}$ dwarf stars. Astrobiology 12:1109-1122.

Grillmair, C.J., Charbonneau, D., Burrows, A., Armus, L., Stauffer, J., Meadows, V., Van Cleve, J., and Levine, D. (2007) A Spitzer spectrum of the exoplanet HD 189733b. Astrophys J 658:L115-L118.

Hamano, K., Kawahara, H., Abe, Y., Onishi, M., and Hashimoto, G.L. (2015) Lifetime and spectral evolution of a magma ocean with a steam atmosphere: its detectability by future direct imaging. Astrophys $J$ 806, doi:10.1088/0004637X/806/2/216.

Hays, J.D., Imbrie, J., and Shackleton, N.J. (1976) Variations in the Earth's orbit: pacemaker of the ice ages. Science 194:1121-1132.

Hedelt, P., von Paris, P., Godolt, M., Gebauer, S., Grenfell, J.L., Rauer, H., Shreier, F., Selsis, F., and Trautmann, T. (2013) Spectral features of Earth-like planets and their detectability at different orbital distances around F, G, and K-type stars. Astron Astrophys 553:A9.

Hegde, S., Paulino-Lima, I.G., Kent, R., Kaltenegger, L., and Rothschild, L. (2015) Surface biosignatures of exo-Earths: remote detection of extraterrestrial life. Proc Natl Acad Sci USA 112:3886-3891.

Heng, K. and Kitzmann, D. (2017) The theory of transmission spectra revisited: a semi-analytical method for interpreting WFC3 data and an unresolved challenge. Mon Not R Astron Soc 470:2972-2981.

Hippke, M. and Angerhausen, D. (2015) Photometry’s bright future: detecting solar system analogs with future space telescopes. Astrophys J 810, doi:10.1088/0004-637X/810/1/29.

Holman, M.J. and Murray, N.W. (2005) The use of transit timing to detect terrestrial-mass extrasolar planets. Science 307:1288-1291.

Hu, R., Ehlmann, B.L., and Seager, S. (2012a) Theoretical spectra of terrestrial exoplanet surfaces. Astrophys $J$ 752, doi: 10.1088/0004-637X/752/1/7.

Hu, R., Seager, S., and Bains, W. (2012b) Photochemistry in terrestrial exoplanet atmospheres. I. Photochemistry model and benchmark cases. Astrophys J 761, doi:10.1088/0004637X/761/2/166.

$\mathrm{Hu}$, R., Seager, S., and Bains, W. (2013) Photochemistry in terrestrial exoplanet atmospheres. II. $\mathrm{H}_{2} \mathrm{~S}$ and $\mathrm{SO}_{2}$ photochemistry in anoxic atmospheres. Astrophys $J$ 769, doi: 10.1088/0004-637X/769/1/6.

Hu, Y. and Yang, J. (2014) Role of ocean heat transport in climates of tidally locked exoplanets around $\mathrm{M}$ dwarf stars. Proc Natl Acad Sci USA 111:629-634.

Huber, D., Chaplin, W.J., Christensen-Dalsgaard, J., Gilliland, R.L., Kjeldsen, H., Buchhave, L.A., Fischer, D.A., Lissauer, J.J., Rowe, J.F., Sanchis-Ojeda, R., Basu, S., Handberg, R., Hekker, S., Howard, A.W., Isaacson, H., Karoff, C., Latham, D.W., Lund, M.N., Lundkvist, M., Marcy, G.W., Miglio, A., Silva Aguirre, V., Stello, D., Arentoft, T., Barclay, T., Bedding, T.R., Burke, C.J., Christiansen, J.L., Elsworth, Y.P.,
Haas, M.R., Kawaler, S.D., Metcalfe, T.S., Mullally, F., and Thompson, S.E. (2013) Fundamental properties of Kepler planet-candidate host stars using asteroseismology. Astrophys $J$ 767, doi:10.1088/0004-637X/767/2/127.

Isaacson, H. and Fischer, D. (2010) Chromospheric activity and jitter measurements for 2630 stars on the California planet search. Astrophys J 725:875-885.

Izidoro, A., Morbidelli, A., and Raymond, S.N. (2014) Terrestrial planet formation in the presence of migrating superEarths. Astrophys J 794, doi:10.1088/0004-637X/794/1/11.

Joshi, M. (2003) Climate model studies of synchronously rotating planets. Astrobiology 3:415-427.

Joshi, M.M., Haberle, R.M., and Reynolds, R.T. (1997) Simulations of the atmospheres of synchronously rotating terrestrial planets orbiting $\mathrm{M}$ dwarfs: conditions for atmospheric collapse and the implications for habitability. Icarus 129: 450-465.

Jura, M. (2004) An observational signature of evolved oceans on extrasolar terrestrial planets. Astrophys $J$ 605: L65-L68.

Kadoya, S. and Tajika, E. (2014) Conditions for oceans on Earth-like planets orbiting within the habitable zone: importance of volcanic $\mathrm{CO}_{2}$ degassing. Astrophys $J$ 790, doi: 10.1088/0004-637X/790/2/107.

Kalas, P., Graham, J.R., Chiang, E., Fitzgerald, M.P., Clampin, M., Kite, E.S., Stapelfeldt, K., Marois, C., and Krist, J. (2008) Optical images of an exosolar planet 25 light-years from Earth. Science 322:1345-1348.

Kaltenegger, L. (2017) How to characterize habitable worlds and signs of life. Annu Rev Astron Astrophys 55:433-485.

Kaltenegger, L. and Sasselov, D. (2010) Detecting planetary geochemical cycles on exoplanets: atmospheric signatures and the case of $\mathrm{SO}_{2}$. Astrophys $J$ 708:1162-1167.

Kaltenegger, L. and Traub, W.A. (2009) Transits of Earth-like planets. Astrophys J 698:519-527.

Kaltenegger, L., Traub, W.A., and Jucks, K.W. (2008) Spectral evolution of an Earth-like planet. Astrophys $J$ 658, doi: $10.1086 / 510996$.

Kaltenegger, L., Henning, W., and Sasselov, D. (2010) Detecting volcanism on extrasolar planets. Astrophys J 140: 1370-1380.

Kaltenegger, L., Sasselov, D., and Rugheimer, S. (2013) Water planets in the habitable zone: atmospheric chemistry, observable features, and the case of Kepler-62e and -62f. Astrophys J 775:L47.

Kane, S.R. (2013) Completeness of imaging surveys for eccentric exoplanets. Astrophys J 766, doi:10.1088/0004-637X/ $766 / 1 / 10$

Kane, S.R. and von Braun, K. (2008) Constraining orbital parameters through planetary transit monitoring. Astrophys $J$ 689:492-498.

Kane, S.R., Mahadevan, S., von Braun, K., Laughlin, G., and Ciardi, D.R. (2009) Refining exoplanet ephemerides and transit observing strategies. Publ Astron Soc Pac 121, doi: $10.1086 / 648564$.

Karalidi, T. and Stam, D.M. (2012) Modeled flux and polarization signals of horizontally inhomogeneous exoplanets applied to Earth-like planets. Astron Astrophys Suppl Ser 546:A56.

Kasting, J.F., Whitmire, D.P., and Reynolds, R.T. (1993) Habitable zones around main sequence stars. Icarus 101:108-128.

Kawahara, H. (2016) Frequency modulation of directly imaged exoplanets: geometric effect as a probe of planetary obliquity. Astrophys $J$ 822, doi:10.3847/0004-637X/822/2/112. 
Kawahara, H. and Fujii, Y. (2010) Global mapping of Earth-like exoplanets from scattered light curves. Astrophys J 720: 1333-1350.

Kawahara, H. and Fujii, Y. (2011) Mapping clouds and terrain of Earth-like planets from photometric variability: demonstration with planets in face-on orbits. Astrophys $J$ 739:L62.

Kawahara, H. and Hirano, T. (2014) Characterizing Earth-like planets using a combination of high-dispersion spectroscopy and high-contrast instruments: Doppler-shifted water and oxygen lines. arXiv:1409.5740. Available online at http:// arxiv.org/abs/1409.5740

Kawahara, H., Matsuo, T., Takami, M., Fujii, Y., Kotani, T., Murakami, N., Tamura, M., and Guyon, O. (2012) Can ground-based telescopes detect the oxygen $1.27 \mu \mathrm{m}$ absorption feature as a biomarker in exoplanets? Astrophys $J$ 758, doi:10.1088/0004-637X/758/1/13.

Kawahara, H., Murakami, N., Matsuo, T., and Kotani, T. (2014) Spectroscopic coronagraphy for planetary radial velocimetry of exoplanets. Astrophys J Suppl Ser 212, doi:10.1088/00670049/212/2/27.

Keller-Rudek, H., Moortgat, G.K., Sander, R., and Sörensen, R. (2013) The MPI-Mainz UV/VIS spectral atlas of gaseous molecules of atmospheric interest. Earth System Science Data 5:365-373.

Kiang, N.Y., Siefert, J., Govindjee, and Blankenship, R.E. (2007a) Spectral signatures of photosynthesis. I. Review of Earth organisms. Astrobiology 7:222-251.

Kiang, N.Y., Segura, A., Tinetti, G., Govindjee, Blankenship, R.E., Cohen, M., Siefert, J., Crisp, D., and Meadows, V.S. (2007b) Spectral signatures of photosynthesis. II. Coevolution with other stars and the atmosphere on extrasolar worlds. Astrobiology 7:252-274.

Knutson, H.A., Charbonneau, D., Allen, L.E., Fortney, J.J., Agol, E., Cowan, N.B., Showman, A.P., Cooper, C.S., and Megeath, S.T. (2007) A map of the day-night contrast of the extrasolar planet HD 189733b. Nature 447:183-186.

Knutson, H.A., Lewis, N., Fortney, J.J., Burrows, A., Showman, A.P., Cowan, N.B., Algol, E., Aigrain, S., Charbonneau, D., Deming, D., Désert, J.-M., Henry, G.W., Langton, J., and Laughlin, G. (2012) 3.6 and $4.5 \mu \mathrm{m}$ phase curves and evidence for non-equilibrium chemistry in the atmosphere of extrasolar planet HD 189733b. Astrophys J 754, doi:10.1088/ 0004-637X/754/1/22.

Konopacky, Q.M., Barman, T.S., Macintosh, B.A., and Marois, C. (2013) Detection of carbon monoxide and water absorption lines in an exoplanet atmosphere. Science 339: 1398-1401.

Kopparapu, R.K. (2013) A revised estimate of the occurrence rate of terrestrial planets in the habitable zones around Kepler M-dwarfs. Astrophys J Lett 767, Issue 1, article id. L8.

Kopparapu, R.K., Ramirez, R., Kasting, J.F., Eymet, V., Robinson, T.D., Mahadevan, S., Terrien, R.C., Domagal-Goldman, S., Meadows, V., and Deshpande, R. (2013) Habitable zones around main-sequence stars: new estimates. Astrophys $J$ 765, doi:10.1088/0004-637X/765/2/131.

Kopparapu, R.K., Ramirez, R.M., SchottelKotte, J., Kasting, J.F., Domagal-Goldman, S., and Eymet, V. (2014) Habitable zones around main-sequence stars: dependence on planetary mass. Astrophys J 787:L29.

Kopparapu, R.K., Wolf, E.T., Arney, G., Batalha, N.E., HaqqMisra, J., Grimm, S.L., and Heng, K. (2017) Habitable moist atmospheres on terrestrial planets near the inner edge of the habitable zone around M dwarfs. Astrophys $J$ 845(1): article id. $5,16 \mathrm{pp}$.
Kraft, R.P. (1967) Studies of stellar rotation. V. The dependence of rotation on age among solar-type stars. Astrophys $J$ 150, doi:10.1086/149359.

Kreidberg, L. and Loeb, A. (2016) Prospects for characterizing the atmosphere of Proxima Centauri b. Astrophys $J$ 832:L12.

Kreidberg, L., Bean, J.L., Désert, J.-M., Benneke, B., Deming, D., Stevenson, K.B., Seager, S., Berta-Thompson, Z., Seifahrt, A., and Homeier, D. (2014) Clouds in the atmosphere of the super-Earth exoplanet GJ 1214b. Nature 505: 69-72.

Krissansen-Totton, J., Schwieterman, E.W., Charnay, B., Arney, G., Robinson, T.D., Meadows, V., and Catling, D.C. (2016) Is the Pale Blue Dot unique? Optimized photometric bands for identifying Earth-like exoplanets. Astrophys $J$ 817, doi:10.3847/0004-637X/817/1/31.

Kuhn, J.R. and Berdyugina, S.V. (2015) Global warming as a detectable thermodynamic marker of Earth-like extrasolar civilizations: the case for a telescope like Colossus. International Journal of Astrobiology 14:401-410.

Kuhn, J.R., Berdyugina, S.V., Langlois, M., Moretto, G., Thiébaut, E., Harlingten, C., and Halliday, D. (2014) Looking beyond 30m-class telescopes: the Colossus project. Proc SPIE 91451, doi:10.1117/12.2056594.

Kuzuhara, M., Tamura, M., Kudo, T., Janson, M., Kandori, R., Brandt, T.D., Thalmann, C., Spiegel, D., Biller, B., Carson, J., Hori, Y., Suzuki, R., Burrows, A., Henning, T., Turner, E.L., McElwain, M.W., Moro-Martín, A., Suenaga, T., Takahashi, Y.H., Kwon, J., Lucas, P., Abe, L., Brandner, W., Egner, S., Feldt, M., Fujiwara, H., Goto, M., Grady, C.A., Guyon, O., Hashimoto, J., Hayano, Y., Hayashi, M., Hayashi, S.S., Hodapp, K.W., Ishii, M., Iye, M., Knapp, G.R., Matsuo, T., Mayama, S., Miyama, S., Morino, J.-I., Nishikawa, J., Nishimura, T., Kotani, T., Kusakabe, N., Pyo, T.-S., Serabyn, E., Suto, H., Takami, M., Takato, N., Terada, H., Tomono, D., Watanabe, M., Wisniewski, J.P., Yamada, T., Takami, H., and Usuda, T. (2013) Direct Imaging of a cold jovian exoplanet in orbit around the Sun-like star GJ 504. Astrophys $J$ 774, doi:10.1088/0004-637X/774/1/11.

Lagrange, A.-M., Bonnefoy, M., Chauvin, G., Apai, D., Ehrenreich, D., Boccaletti, A., Gratadour, D., Rouan, D., Mouillet, D., Lacour, S., and Kasper, M. (2010) A giant planet imaged in the disk of the young star $\beta$ Pictoris. Science 329:57-59.

Laskar, J. and Robutel, P. (1993) The chaotic obliquity of the planets. Nature 361:608-612.

Lawson, P.R., Lay, O.P., Johnston, K.J., and Beichman, C.A. (2007) Terrestrial Planet Finder Interferometer Science Working Group Report, JPL Publication 07-1, Jet Propulsion Laboratory, California Institute of Technology, Pasadena, CA.

Lawson, P.R., Poyneer, L., Barrett, H., Frazin, R., Caucci, L., Devaney, N., Furenlid, L., Gladysz, S., Guyon, O., Krist, J., Maire, J., Marois, C., Mawet, D., Mouillet, D., Mugnier, L., Pearson, I., Perrin, M., Pueyo, L., and Savransky, D. (2012) Adaptive Optics Systems III. Proc SPIE 8447, article id. 844722, 21 pp.

Leconte, J., Forget, F., Charnay, B., Wordsworth, R., and Pottier, A. (2013) Increased insolation threshold for runaway greenhouse processes on Earth-like planets. Nature 504:268-271.

Leconte, J., Forget, F., and Lammer, H. (2015) On the (anticipated) diversity of terrestrial planet atmospheres. Experimental Astronomy 40:449-467. 
Léger, A., Mariotti, J.M., Mennesson, B., Ollivier, M., Puget, J.L., Rouan, D., and Schneider, J. (1996) Could we search for primitive life on extrasolar planets in the near future? Icarus 123:249-255.

Léger, A., Selsis, F., Sotin, C., Guillot, T., Despois, D., Mawet, D., Ollivier, M., Labèque, A., Valette, C., Brachet, F., Chazelas, B., and Lammer, H. (2004) A new family of planets? "Ocean-planets." Icarus 169:499-504.

Lenardic, A. and Crowley, J.W. (2012) On the notion of welldefined tectonic regimes for terrestrial planets in this solar system and others. Astrophys J 755, doi:10.1088/0004-637X/ $755 / 2 / 132$.

Levine, M., Lisman, D., Shaklan, S., Kasting, J., Traub, W., Alexander, J., Angel, R., Blaurock, C., Brown, M., Brown, R., Burrows, C., Clampin, M., Cohen, E., Content, D., Dewell, L., Dumont, P., Egerman, R., Ferguson, H., Ford, V., Greene, J., Guyon, O., Hammel, H., Heap, S., Ho, T., Horner, S., Hunyadi, S., Irish, S., Jackson, C., Kasdin, J., Kissil, A., Krim, M., Kuchner, M., Kwack, E., Lillie, C., Lin, D., Liu, A., Marchen, L., Marley, M., Meadows, V., Mosier, G., Mouroulis, P., Noecker, M., Ohl, R., Oppenheimer, B., Pitman, J., Ridgway, S., Sabatke, E., Seager, S., Shao, M., Smith, A., Soummer, R., Stapelfeldt, K., Tenerell, D., Trauger, J., and Vanderbei, R. (2009) Terrestrial Planet Finder Coronagraph (TPF-C) flight baseline concept. arXiv: 0911.3200. Available online at http://arxiv.org/abs/0911.3200

Line, M.R. and Parmentier, V. (2016) The influence of nonuniform cloud cover on transit transmission spectra. Astrophys $J$ 820, doi:10.3847/0004-637X/820/1/78.

Line, M.R., Wolf, A.S., Zhang, X., Knutson, H., Kammer, J.A., Ellison, E., Deroo, P., Crisp, D., and Yung, Y.L. (2013) A systematic retrieval analysis of secondary eclipse spectra. I. A comparison of atmospheric retrieval techniques. Astrophys $J$ 775, doi:10.1088/0004-637X/775/2/137.

Lissauer, J.J., Fabrycky, D.C., Ford, E.B., Borucki, W.J., Fressin, F., Marcy, G.W., Orosz, J.A., Rowe, J.F., Torres, G., Welsh, W.F., Batalha, N.M., Bryson, S.T., Buchhave, L.A., Caldwell, D.A., Carter, J.A., Charbonneau, D., Christiansen, J.L., Cochran, W.D., Desert, J.-M., Dunham, E.W., Fanelli, M.N., Fortney, J.J., Gautier, T.N., III, Geary, J.C., Gilliland, R.L., Haas, M.R., Hall, J.R., Holman, M.J., Koch, D.G., Latham, D.W., Lopez, E., McCauliff, S., Miller, N., Morehead, R.C., Quintana, E.V., Ragozzine, D., Sasselov, D., Short, D.R., and Steffen, J.H. (2011) A closely packed system of low-mass, low-density planets transiting Kepler-11. Nature 470:53-58.

Livengood, T.A., Deming, L.D., A'Hearn, M.F., Charbonneau, D., Hewagama, T., Lisse, C.M., McFadden, L.A., Meadows, V.S., Robinson, T.D., Seager, S., and Wellnitz, D.D. (2011) Properties of an Earth-like planet orbiting a Sun-like star: Earth observed by the EPOXI Mission. Astrobiology 11:907-930.

Loeb, A. and Maoz, D. (2013) Detecting biomarkers in habitable-zone earths transiting white dwarfs. Mon Not $R$ Astron Soc 432:11-15.

Lovis, C., Snellen, I., Mouillet, D., Pepe, F., Wildi, F., Astudillo-Defru, N., Beuzit, J.-L., Bonfils, X., Cheetham, A., Conod, U., Delfosse, X., Ehrenreich, D., Figueira, P., Forveille, T., Martins, J.H.C., Quanz, S., Santos, N.C., Schmid, H.-M., Ségransan, D., and Udry, S. (2017) Atmospheric characterization of Proxima b by coupling the SPHERE highcontrast imager to the ESPRESSO spectrograph. Astron Astrophys 599:A16.

Loyd, R.O.P., France, K., Youngblood, A., Schneider, P.C., Brown, A., Hu, R., Linsky, J.L., Froning, C.S., Redfield, S.,
Rugheimer, S., and Tian, F. (2016) The MUSCLES Treasury Survey. III. X-ray to infrared spectra of $11 \mathrm{M}$ and K stars hosting planets. Astrophys J 824, doi:10.3847/0004-637X/824/2/102.

Luger, R. and Barnes, R. (2015) Extreme water loss and abiotic $\mathrm{O}_{2}$ buildup on planets throughout the habitable zones of $\mathrm{M}$ dwarfs. Astrobiology 15:119-143.

Macintosh, B., Graham, J.R., Barman, T., De Rosa, R.J., Konopacky, Q., Marley, M.S., Marois, C., Nielsen, E.L., Pueyo, L., Rajan, A., Rameau, J., Saumon, D., Wang, J.J., Patience, J., Ammons, M., Arriaga, P., Artigau, E., Beckwith, S., Brewster, J., Bruzzone, S., Bulger, J., Burningham, B., Burrows, A.S., Chen, C., Chiang, E., Chilcote, J.K., Dawson, R.I., Dong, R., Doyon, R., Draper, Z.H., Duchêne, G., Esposito, T.M., Fabrycky, D., Fitzgerald, M.P., Follette, K.B., Fortney, J.J., Gerard, B., Goodsell, S., Greenbaum, A.Z., Hibon, P., Hinkley, S., Cotten, T.H., Hung, L.-W., Ingraham, P., Johnson-Groh, M., Kalas, P., Lafreniere, D., Larkin, J.E., Lee, J., Line, M., Long, D., Maire, J., Marchis, F., Matthews, B.C., Max, C.E., Metchev, S., Millar-Blanchaer, M.A., Mittal, T., Morley, C.V., Morzinski, K.M., Murray-Clay, R., Oppenheimer, R., Palmer, D.W., Patel, R., Perrin, M.D., Poyneer, L.A., Rafikov, R.R., Rantakyrö, F.T., Rice, E.L., Rojo, P., Rudy, A.R., Ruffio, J.-B., Ruiz, M.T., Sadakuni, N., Saddlemyer, L., Salama, M., Savransky, D., Schneider, A.C., Sivaramakrishnan, A., Song, I., Soummer, R., Thomas, S., Vasisht, G., Wallace, J.K., Ward-Duong, K., Wiktorowicz, S.J., Wolff, S.G., and Zuckerman, B. (2015) Discovery and spectroscopy of the young jovian planet 51 Eri b with the Gemini Planet Imager. Science 350:64-67.

Maehara, H., Shibayama, T., Notsu, S., Notsu, Y., Nagao, T., Kusaba, S., Honda, S., Nogami, D., and Shibata, K. (2012) Superflares on solar-type stars. Nature 485:478-481.

Maehara, H., Shibayama, T., Notsu, Y., Notsu, S., Honda, S., Nogami, D., and Shibata, K. (2015) Statistical properties of superflares on solar-type stars based on 1-min cadence data. Earth Planets Space 67, doi:10.1186/s40623-015-0217-z.

Mahadevan, S., Ramsey, L., Bender, C., Terrien, R., Wright, J.T., Halverson, S., Hearty, F., Nelson, M., Burton, A., Redman, S., Osterman, S., Diddams, S., Kasting, J., Endl, M., and Deshpande, R. (2012) The habitable-zone planet finder: a stabilized fiber-fed NIR spectrograph for the Hobby-Eberly Telescope. Proc SPIE 8446, doi:10.1117/12.926102.

Majeau, C., Agol, E., and Cowan, N.B. (2012) A two-dimensional infrared map of the extrasolar planet HD 189733b. Astrophys $J$ 747:L20.

Marois, C., Macintosh, B., Barman, T., Zuckerman, B., Song, I., Patience, J., Lafrenière, D., and Doyon, R. (2008) Direct imaging of multiple planets orbiting the star HR 8799. Science 322:1348-1352.

Martins, J.H.C., Santos, N.C., Figueira, P., Faria, J.P., Montalto, M., Boisse, I., Ehrenreich, D., Lovis, C., Mayor, M., Melo, C., Pepe, F., Sousa, S.G., Udry, S., and Cunha, D. (2015) Evidence for a spectroscopic direct detection of reflected light from 51 Pegasi b. Astron Astrophys 576:A134.

Matsuo, T., Itoh, S., Shibai, H., Sumi, T., and Yamamuro, T. (2016) A new concept for spectrophotometry of exoplanets with space-borne telescopes. Astrophys $J$ 823, Issue 2, article id. 139,12 pp.

Maurin, A.S., Selsis, F., Hersant, F., and Belu, A. (2012) Thermal phase curves of nontransiting terrestrial exoplanets. II. Characterizing airless planets. Astron Astrophys 538, doi: 10.1051/0004-6361/201117054.

Mayor, M. and Queloz, D. (1995) A Jupiter-mass companion to a solar-type star. Nature 378:355-359. 
McCullough, P.R. (2006) Models of polarized light from oceans and atmospheres of Earth-like extrasolar planets. arXiv:astro$\mathrm{ph} / 0610518$. Available online at http://arxiv.org/abs/astro-ph/ 0610518

Meadows, V.S., Reinhard, C.T., Arney, G.N., Parenteau, M.N., Schwieterman, E.W., Domagal-Goldman, S., Lincowski, A.P., Stapelfeldt, K.R., Rauer, H., DasSarma, S., Hegde, S., Narita, N., Deitrick, R., Lustig-Yaeger, J., Lyons, T.W., Siegler, N., and Grenfell, J.L. (2018) Exoplanet biosignatures: understanding oxygen as a biosignature in the context of its environment. Astrobiology 18:630-662.

Meixner, M., Cooray, A., Carter, R., DiPirro, M., Flores, A., Leisawitz, D., Armus, L., Battersby, C., Bergin, E., Bradford, C.M., Ennico, K., Melnick, G.J., Milam, S., Narayanan, D., Pontoppidan, K., Pope, A., Roellig, T., Sandstrom, K., Su, K.Y.L., Vieira, J., Wright, E., Zmuidzinas, J., Alato, S., Carey, S., Gerin, M., Helmich, F., Menten, K., Scott, D., Sakon, I., and Vavrek, R. (2016) The Far-Infrared Surveyor Mission study: Paper I, the genesis. Proc SPIE 9904, id. 99040K 8 pp.

Merienne, M.F., Jenouvrier, A., Coquart, B., and Lux, J.P. (1997) The $\mathrm{NO}_{2}$ absorption spectrum. IV: The 200-400 nm region at $220 \mathrm{~K}$. J Atmos Chem 27:219-232.

Miles-Páez, P.A., Pallé, E., and Zapatero Osorio, M.R. (2014) Simultaneous optical and near-infrared linear spectropolarimetry of the Earthshine. Astron Astrophys 562:L5.

Miller-Ricci, E., Seager, S., and Sasselov, D. (2009) The atmospheric signatures of super-Earths: how to distinguish between hydrogen-rich and hydrogen-poor atmospheres. Astrophys $J$ 690, doi:10.1088/0004-637X/690/2/1056.

Misra, A., Meadows, V., Claire, M., and Crisp, D. (2014a) Using dimers to measure biosignatures and atmospheric pressure for terrestrial exoplanets. Astrobiology 14:67-86.

Misra, A., Meadows, V., and Crisp, D. (2014b) The effects of refraction on transit transmission spectroscopy: application to Earth-like exoplanets. Astrophys J 792, doi:10.1088/0004637X/792/1/61.

Montañés-Rodríguez, P., Pallé, E., Goode, P.R., and MartínTorres, F.J. (2006) Vegetation signature in the observed globally integrated spectrum of Earth considering simultaneous cloud data: applications for extrasolar planets. Astrophys $J$ 651, doi:10.1086/507694.

Mordasini, C., Alibert, Y., Benz, W., Klahr, H., and Henning, T. (2012) Extrasolar planet population synthesis. IV. Correlations with disk metallicity, mass, and lifetime. Astron Astrophys 541:A97.

Morton, T.D. and Swift, J. (2014) The radius distribution of planets around cool stars. Astrophys J 791, doi:10.1088/0004$637 X / 791 / 1 / 10$

Nettelmann, N., Kramm, U., Redmer, R., and Neuhäuser, R. (2010) Interior structure models of GJ 436b. Astron Astrophys 523:A26.

Nettelmann, N., Fortney, J.J., Kramm, U., and Redmer, R. (2011) Thermal evolution and structure models of the transiting super-Earth GJ 1214b. Astrophys J 733, doi:10.1088/ 0004-637X/733/1/2.

Noyes, R.W., Hartmann, L.W., Baliunas, S.L., Duncan, D.K., Vaughan, A.H. (1984) Rotation, convection, and magnetic activity in lower main-sequence stars. Astrophys $J$ 279, doi: $10.1086 / 161945$

Oakley, P.H.H. and Cash W. (2009) Construction of an Earth model: analysis of exoplanet light curves and mapping the next Earth with the New Worlds Observer. Astrophys J 700: $1428-1439$.
Öberg, K.I., Murray-Clay, R., and Bergin, E.A. (2011) The effects of snowlines on $\mathrm{C} / \mathrm{O}$ in planetary atmospheres. Astrophys J 743:L16.

Pallavicini, R., Golub, L., Rosner, R., Vaiana, G.S., Ayres, T., and Linsky, J.L. (1981) Relations among stellar X-rayemission observed from Einstein, stellar rotation and bolometric luminosity. Astrophys J 248:279-290.

Pallé, E., Ford, E.B., Seager, S., Montanes-Rodriguez, P., and Vazquez, M. (2008) Identifying the rotation rate and the presence of dynamic weather on extrasolar Earth-like planets from photometric observations. Astrophys J 676:1319-1329.

Pallé, E., Osorio, M.R.Z., Barrena, R., Montañés-Rodríguez, P., and Martín, E.L. (2009) Earth's transmission spectrum from lunar eclipse observations. Nature 459:814-816.

Pallé, E., Zapatero Osorio, M.R., and García Muñoz, A. (2011) Characterizing the atmospheres of transiting rocky planets around late-type dwarfs. Astrophys J 728, doi:10.1088/0004637X/728/1/19.

Pepe, F., Molaro, P., Cristiani, S., Rebolo, R., Santos, N.C., Dekker, H., Mégevand, D., Zerbi, F.M., Cabral, A., Di Marcantonio, P., Abreu, M., Affolter, M., Aliverti, M., Allende Prieto, C., Amate, M., Avila, G., Baldini, V., Bristow, P., Broeg, C., Cirami, R., Coelho, J., Conconi, P., Coretti, I., Cupani, G., D’Odorico, V., De Caprio, V., Delabre, B., Dorn, R., Figueira, P., Fragoso, A., Galeotta, S., Genolet, L., Gomes, R., González Hernández, J.I., Hughes, I., Iwert, O., Kerber, F., Landoni, M., Lizon, J.-L., Lovis, C., Maire, C., Mannetta, M., Martins, C., Monteiro, M., Oliveira, A., Poretti, E., Rasilla, J.L., Riva, M., Santana Tschudi, S., Santos, P., Sosnowska, D., Sousa, S., Spanó, P., Tenegi, F., Toso, G., Vanzella, E., Viel, M., and Zapatero Osorio, M.R. (2014) ESPRESSO: The next European exoplanet hunter. arXiv:1401.5918. Available online at http://arxiv.org/abs/ 1401.5918

Perryman, M., Hartman, J., Bakos, G.Á., and Lindegren, L. (2014) Astrometric exoplanet detection with Gaia. Astrophys J 797, doi:10.1088/0004-637X/797/1/14.

Petigura, E.A., Howard, A.W., and Marcy, G.W. (2013) Prevalence of Earth-size planets orbiting Sun-like stars. Proc Natl Acad Sci USA 110:19273-19278.

Pierrehumbert, R. and Gaidos, E. (2011) Hydrogen greenhouse planets beyond the habitable zone. Astrophys J Lett 734:L13.

Pinhas, A., Madhusudhan, N., and Clarke, C. (2016) Efficiency of planetesimal ablation in giant planetary envelopes. Mon Not R Astron Soc 463:4516-4532.

Postman, M., Argabright, V., Arnold, B., Aronstein, D., Atcheson, P., Blouke, M., Brown, T., Calzetti, D., Cash, W., Clampin, M., Content, D., Dailey, D., Danner, R., Doxsey, R., Ebbets, D., Eisenhardt, P., Feinberg, L., Fruchter, A., Mauro Giavalisco, M., Glassman, T., Gong, Q., Green, J., Grunsfeld, J., Gull, T., Hickey, G., Hopkins, R., Hraba, J., Hyde, T., Jordan, I., Kasdin, J., Kendrick, S., Kilston, S., Koekemoer, A., Korechoff, B., Krist, J., Mather, J., Lillie, C., Lo, A., Lyon, R., McCullough, P., Mosier, G., Mountain, M., Oegerle, B., Pasquale, B., Purves, L., Penera, C., Polidan, R., Redding, D., Sahu, K., Saif, B., Sembach, K., Shull, M., Smith, S., Sonneborn, G., Spergel, D., Stahl, P., Stapelfeldt, K., Thronson, H., Thronton, G., Townsend, J., Traub, W., Unwin, S., Valenti, J., Vanderbei, R., Werner, M., Wesenberg, R., Wiseman, J., and Woodgate, B. (2009) Advanced Technology Large-Aperture Space Telescope (ATLAST): a technology roadmap for the next decade. arXiv:0904.0941. Available online at http://arxiv.org/abs/0904.0941 
Queloz, D., Henry, G.W., Sivan, J.P., Baliunas, S.L., Beuzit, J.L., Donahue, R.A., Mayor, M., Naef, D., Perrier, C., and Udry, S. (2001) No planet for HD 166435. Astron Astrophys 379:279-287.

Quirrenbach, A., Amado, P.J., Caballero, J.A., Mundt, R., Reiners, A., Ribas, I., Seifert, W., Abril, M., Aceituno, J., Alonso-Floriano, F.J., Anwand-Heerwart, H., Azzaro, M., Bauer, F., Barrado, D., Becerril, S., Bejar, V.J.S., Benitez, D., Berdinas, Z.M., Brinkmöller, M., Cardenas, M.C., Casal, E., Claret, A., Colomé, J., Cortes-Contreras, M., Czesla, S., Doellinger, M., Dreizler, S., Feiz, C., Fernandez, M., Ferro, I.M., Fuhrmeister, B., Galadi, D., Gallardo, I., Gálvez-Ortiz, M.C., Garcia-Piquer, A., Garrido, R., Gesa, L., Gómez Galera, V., González Hernández, J.I., Gonzalez Peinado, R., Grözinger, U., Guàrdia, J., Guenther, E.W., de Guindos, E., Hagen, H.-J., Hatzes, A.P., Hauschildt, P.H., Helmling, J., Henning, T., Hermann, D., Hernández Arabi, R., Hernández Castaño, L., Hernández Hernando, F., Herrero, E., Huber, A., Huber, K.F., Huke, P., Jeffers, S.V., de Juan, E., Kaminski, A., Kehr, M., Kim, M., Klein, R., Klüter, J., Kürster, M., Lafarga, M., Lara, L.M., Lamert, A., Laun, W., Launhardt, R., Lemke, U., Lenzen, R., Llamas, M., Lopez del Fresno, M., López-Puertas, M., López-Santiago, J., Lopez Salas, J.F., Magan Madinabeitia, H., Mall, U., Mandel, H., Mancini, L., Marin Molina, J.A., Maroto Fernández, D., Martín, E.L., Martín-Ruiz, S., Marvin, C., Mathar, R.J., Mirabet, E., Montes, D., Morales, J.C., Morales Muñoz, R., Nagel, E., Naranjo, V., Nowak, G., Palle, E., Panduro, J., Passegger, V.M., Pavlov, A., Pedraz, S., Perez, E., Pérez-Medialdea, D., Perger, M., Pluto, M., Ramón, A., Rebolo, R., Redondo, P., Reffert, S., Reinhart, S., Rhode, P., Rix, H.-W., Rodler, F., Rodríguez, E., Rodríguez López, C., Rohloff, R.R., Rosich, A., Sanchez Carrasco, M.A., Sanz-Forcada, J., Sarkis, P., Sarmiento, L.F., Schäfer, S., Schiller, J., Schmidt, C., Schmitt, J.H.M.M., Schöfer, P., Schweitzer, A., Shulyak, D., Solano, E., Stahl, O., Storz, C., Tabernero, H.M., Tala, M., Tal-Or, L., Ulbrich, R.-G., Veredas, G., Vico Linares, J.I., Vilardell, F., Wagner, K., Winkler, J., Zapatero Osorio, M.-R., Zechmeister, M., Ammler-von Eiff, M., AngladaEscudé, G., del Burgo, C., Garcia-Vargas, M.L., Klutsch, A., Lizon, J.-L., Lopez-Morales, M., Ofir, A., Pérez-Calpena, A., Perryman, M.A.C., Sánchez-Blanco, E., Strachan, J.B.P., Stürmer, J., Suárez, J.C., Trifonov, T., Tulloch, S.M., and Xu, W. (2016) CARMENES: an overview six months after first light. Proc SPIE 9908, doi:10.1117/12.2231880.

Ramirez, R.M. and Kaltenegger, L. (2014) The habitable zones of pre-main-sequence stars. Astrophys J 797:L25.

Ramirez, R.M. and Kaltenegger, L. (2016) Habitable zones of post-main sequence stars. Astrophys $J$ 823, doi:10.3847/0004637X/823/1/6.

Ramirez, R.M. and Kaltenegger, L. (2017) A volcanic hydrogen habitable zone. Astrophys J 837:L4.

Rauer, H., Gebauer, S., von Paris, P., Cabrera, J., Godolt, M., Grenfell, J.L., Belu, A., Selsis, F., Hedelt, P., and Schreier, F. (2011) Potential biosignatures in super-Earth atmospheres. I. Spectral appearance of super-Earths around M dwarfs. Astron Astrophys 529:A8.

Rauer, H., Catala, C., Aerts, C., Appourchaux, T., Benz, W., Brandeker, A., Christensen-Dalsgaard, J., Deleuil, M., Gizon, L., Goupil, M.-J., Güdel, M., Janot-Pacheco, E., Mas-Hesse, M., Pagano, I., Piotto, G., Pollacco, D., Santos, Cं., Smith, A., Suárez, J.-C., Szabó, R., Udry, S., Adibekyan, V., Alibert, Y., Almenara, J.-M., Amaro-Seoane, P., Eiff, M.A., Asplund, M., Antonello, E., Barnes, S., Baudin, F., Belkacem, K., Bergemann, M., Bihain, G.,
Birch, A.C., Bonfils, X., Boisse, I., Bonomo, A.S., Borsa, F., Brandão, I.M., Brocato, E., Brun, S., Burleigh, M., Burston, R., Cabrera, J., Cassisi, S., Chaplin, W., Charpinet, S., Chiappini, C., Church, R.P., Csizmadia, Sz., Cunha, M., Damasso, M., Davies, M.B., Deeg, H.J., Díaz, R.F., Dreizler, S., Dreyer, C., Eggenberger, P., Ehrenreich, D., Eigmüller, P., Erikson, A., Farmer, R., Feltzing, S., de Oliveira Fialho, F., Figueira, P., Forveille, T., Fridlund, M., García, R.A., Giommi, P., Giuffrida, G., Godolt, M., Gomes da Silva, J., Granzer, T., Grenfell, J.L., Grotsch-Noels, A., Günther, E., Haswell, C.A., Hatzes, A.P., Hébrard, G., Hekker, S., Helled, R., Heng, K., Jenkins, J.M., Johansen, A., Khodachenko, M.L., Kislyakova, K.G., Kley, W., Kolb, U., Krivova, N., Kupka, F., Lammer, H., Lanza, A.F., Lebreton, Y., Magrin, D., MarcosArenal, P., Marrese, P.M., Marques, J.P., Martins, J., Mathis, S., Mathur, S., Messina, S., Miglio, A., Montalban, J., Montalto, M., Monteiro, M.J.P.F.G., Moradi, H., Moravveji, E., Mordasini, C., Morel, T., Mortier, A., Nascimbeni, V., Nelson, R.P., Nielsen, M.B., Noack, L., Norton, A.J., Ofir, A., Oshagh, M., Ouazzani, R.-M., Pápics, P., Parro, V.C., Petit, P., Plez, B., Poretti, E., Quirrenbach, A., Ragazzoni, R., Raimondo, G., Rainer, M., Reese, D.R., Redmer, R., Reffert, S., Rojas-Ayala, B., Roxburgh, I.W., Salmon, S., Santerne, A., Schneider, J., Schou, J., Schuh, S., Schunker, H., Silva-Valio, A., Silvotti, R., Skillen, I., Snellen, I., Sohl, F., Sousa, S.G., Sozzetti, A., Stello, D., Strassmeier, K.G., Švanda, M., Szabó, Gy.M., Tkachenko, A., Valencia, D., Van Grootel, V., Vauclair, S.D., Ventura, P., Wagner, F.W., Walton, N.A., Weingrill, J., Werner, S.C., Wheatley, P.J., and Zwintz, K. (2014) The PLATO 2.0 mission. Experimental Astronomy 38: 249-330.

Raymond, S.N., Mandell, A.M., and Sigurdsson, S. (2006) Exotic Earths: forming habitable worlds with giant planet migration. Science 313:1413-1416.

Redfield, S., Endl, M., Cochran, W.D., and Koesterke, L. (2008) Sodium absorption from the exoplanetary atmosphere of HD $189733 \mathrm{~b}$ detected in the optical transmission spectrum. Astrophys J 673:L87.

Riaud, P. and Schneider, J. (2007) Improving Earth-like planets' detection with an ELT: the differential radial velocity experiment. Astron Astrophys 469:355-361.

Richardson, L.J., Deming, D., Horning, K., Seager, S., and Harrington J. (2007) A spectrum of an extrasolar planet. Nature 445:892-895.

Ricker, G.R., Winn, J.N., Vanderspek, R., Latham, D.W., Bakos, G.Á., Bean, J.L., Berta-Thompson, Z.K., Brown, T.M., Buchhave, L., Butler, N.R., Butler, R.P., Chaplin, W.J., Charbonneau, D., Christensen-Dalsgaard, J., Clampin, M., Deming, D., Doty, J., De Lee, N., Dressing, C., Dunham, E.W., Endl, M., Fressin, F., Ge, J., Henning, T., Holman, M.J., Howard, A.W., Ida, S., Jenkins, J.M., Jernigan, G., Johnson, J.A., Kaltenegger, L., Kawai, N., Kjeldsen, H., Laughlin, G., Levine, A.M., Lin, D., Lissauer, J.J., MacQueen, P., Marcy, G., McCullough, P.R., Morton, T.D., Narita, N., Paegert, M., Palle, E., Pepe, F., Pepper, J., Quirrenbach, A., Rinehart, S.A., Sasselov, D., Sato, B., Seager, S., Sozzetti, A., Stassun, K.G., Sullivan, P., Szentgyorgyi, A., Torres, G., Udry, S., and Villasenor, J. (2014) Transiting Exoplanet Survey Satellite (TESS). Proc SPIE 9143, doi:10.1117/12.2063489.

Robinson, T.D. (2017) Characterizing exoplanets for habitability. arXiv:1701.05205. Available online at http://arxiv .org/abs/1701.05205

Robinson, T.D. and Crisp, D. (2018) Linearized Flux Evolution (LiFE): A technique for rapidly adapting fluxes from fullphysics radiative transfer models. J Quant Spectrosc Radiat Transf 211:78-95. 
Robinson, T.D., Meadows, V.S., and Crisp, D. (2010) Detecting oceans on extrasolar planets using the glint effect. Astrophys J 721:L67-L71.

Robinson, T.D., Meadows, V.S., Crisp, D., Deming, D., A'Hearn, M.F., Charbonneau, D., and Hewagama, T. (2011) Earth as an extrasolar planet: Earth model validation using EPOXI Earth observations. Astrobiology 11: 393-408.

Robinson, T.D., Ennico, K., Meadows, V.S., Sparks, W., Bussey, D.B.J., Schwieterman, E.W., and Breiner, J. (2014a) Detection of ocean glint and ozone absorption using LCROSS Earth observations. Astrophys J 787, doi:10.1088/0004-637X/ $787 / 2 / 171$

Robinson, T.D., Maltagliati, L., Marley, M.S., and Fortney, J.J. (2014b) Titan solar occultation observations reveal transit spectra of a hazy world. Proc Natl Acad Sci USA 111:90429047.

Robinson, T.D., Stapelfeldt, K.R., and Marley, M.S. (2016) Characterizing rocky and gaseous exoplanets with $2 \mathrm{~m}$ class space-based coronagraphs. Publ Astron Soc Pac 128, Issue 960, pp. 025003.

Rocchetto, M., Waldmann, I.P., Venot, O., Lagage, P.O., and Tinetti, G. (2016) Exploring biases of atmospheric retrievals in simulated JWST transmission spectra of hot Jupiters. Astrophys $J$ 833, doi:10.3847/1538-4357/833/1/120.

Rodler, F. and López-Morales, M. (2014) Feasibility studies for the detection of $\mathrm{O}_{2}$ in an Earth-like exoplanet. Astrophys $J$ 781, doi:10.1088/0004-637X/781/1/54.

Rogers, L.A. (2015) Most 1.6 Earth-radius planets are not rocky. Astrophys J 801, doi:10.1088/0004-637X/801/1/41.

Rogers, L.A. and Seager, S. (2010) A framework for quantifying the degeneracies of exoplanet interior compositions. Astrophys J 712:974-991.

Rothman, L.S., Gordon, I.E., Babikov, Y., Barbe, A., Chris Benner, D., Bernath, P.F., Birk, M., Bizzocchi, L., Boudon, V., Brown, L.R., Campargue, A., Chance, K., Cohen, E.A., Coudert, L.H., Devi, V.M., Drouin, B.J., Fayt, A., Flaud, J.-M., Gamache, R.R., Harrison, J.J., Hartmann, J.-M., Hill, C., Hodges, J.T., Jacquemart, D., Jolly, A., Lamouroux, J., Le Roy, R.J., Li, G., Long, D.A., Lyulin, O.M., Mackie, C.J., Massie, S.T., Mikhailenko, S., Müller, H.S.P., Naumenko, O.V., Nikitin, A.V., Orphal, J., Perevalov, V., Perrin, A., Polovtseva, E.R., Richard, C., Smith, M.A.H., Starikova, E., Sung, K., Tashkun, S., Tennyson, J., Toon, G.C., Tyuterev, Vl.G., and Wagner, G. (2013) The hitran2012 molecular spectroscopic database. J Quant Spectrosc Radiat Transf 130:4-50.

Rugheimer, S., Kaltenegger, L., Zsom, A., Segura, A., and Sasselov, D. (2013) Spectral fingerprints of Earth-like planets around FGK stars. Astrobiology 13:251-269.

Rugheimer, S., Kaltenegger, L., Segura, A., Linsky, J., and Mohanty, S. (2015) Effect of UV radiation on the spectral fingerprints of Earth-like planets orbiting M stars. Astrophys $J$ 809, doi:10.1088/0004-637X/809/1/57.

Saar, S.H. and Fischer, D. (2000) Correcting radial velocities for long-term magnetic activity variations. Astrophys $J$ 534: L105.

Saar, S.H., Butler, R.P., and Marcy, G.W. (1998) Magnetic activity-related radial velocity variations in cool stars: first results from the Lick Extrasolar Planet Survey. Astrophys $J$ 498:L153-L157.

Sachkov, M., Shustov, B., and Gómez de Castro, A.I. (2014) WSO-UV project. Adv Space Res 53:990-995.

Sanromá, E., Pallé, E., Parenteau, M.N., Kiang, N.Y., GutiérrezNavarro, A.M., López, R., and Montañes-Rodríguez, P. (2014)
Characterizing the purple Earth: modeling the globally integrated spectral variability of the Archean Earth. Astrophys $J$ 780, doi:10.1088/0004-637X/780/1/52.

Schwartz, J.C., Sekowski, C., Haggard, H.M., Pallé, E., and Cowan, N.B. (2016) Inferring planetary obliquity using rotational and orbital photometry. Mon Not R Astron Soc 457:926-938.

Schwieterman, E.W., Cockell, C.S., and Meadows, V.S. (2015) Nonphotosynthetic pigments as potential biosignatures. Astrobiology 15:341-361.

Schwieterman, E.W., Meadows, V.S., Domagal-Goldman, S.D., Deming, L.D., Arney, G.N., Luger, R., Harman, C.E., Misra, A., and Barnes, R. (2016) Identifying planetary biosignature impostors: spectral features of $\mathrm{CO}$ and $\mathrm{O}_{4}$ resulting from abiotic $\mathrm{O}_{2} / \mathrm{O}_{3}$ production. Astrophys $J$ 819:L13.

Schwieterman, E.W., Kiang, N.Y., Parenteau, M.N., Harman, C.E., DasSarma, S., Fisher, T.M., Arney, G.N., Hartnett, H.E., Reinhard, C.T., Olson, S.L., Meadows, V.S., Cockell, C.S., Walker, S.I., Grenfell, J.L., Hegde, S., Rugheimer, S., Hu, R., and Lyons, T.W. (2018) Exoplanet biosignatures: a review of remotely detectable signs of life. Astrobiology 18 : 663-708.

Seager, S. and Sasselov, D.D. (2000) Theoretical transmission spectra during extrasolar giant planet transits. Astrophys $J$ 537:916-921.

Seager, S., Turner, E.L., Schafer, J., and Ford, E.B. (2005) Vegetation's red edge: a possible spectroscopic biosignature of extraterrestrial plants. Astrobiology 5:372-390.

Seager, S., Kuchner, M., Hier-Majumder, C.A., and Militzer, B. (2007) Mass-radius relationships for solid exoplanets. Astrophys $J$ 669:1279-1297.

Seager, S., Bains, W., and Hu, R. (2013) Biosignature gases in $\mathrm{H}_{2}$-dominated atmospheres on rocky exoplanets. Astrophys $J$ 777, doi:10.1088/0004-637X/777/2/95.

Seager, S., Turnbull, M., Sparks, W., Thomson, M., Shaklan, S.B., Roberge, A., Kuchner, M., Kasdin, N.J., DomagalGoldman, S., Cash, W., Warfield, K., Lisman, D., Scharf, D., Webb, D., Trabert, R., Martin, S., Cady, E., and Heneghan, C. (2015) The Exo-S probe class starshade mission. Proc SPIE 96050W, doi:10.1117/12.2190378.

Segura, A., Kasting, J.F., Meadows, V., Cohen, M., Scalo, J., Crisp, D., Butler, R.A.H., and Tinetti, G. (2005) Biosignatures from Earth-like planets around M dwarfs. Astrobiology 5:706-725.

Selsis, F., Kaltenegger, L., and Paillet, J. (2008) Terrestrial exoplanets: diversity, habitability and characterization. Physica Scripta 130, Issue, id. 014032.

Selsis, F., Wordsworth, R.D., and Forget, F. (2011) Thermal phase curves of nontransiting terrestrial exoplanets-I. Characterizing atmospheres. Astron Astrophys 532:A1.

Selsis, F., Maurin, A.S., Hersant, F., Leconte, J., Bolmont, E., Raymond, S.N., and Delbo', M. (2013) The effect of rotation and tidal heating on the thermal lightcurves of super Mercuries. Astron Astrophys 555, id. A51, 15 pp.

Selwyn, G., Podolske, J., and Johnston, H.S. (1977) Nitrous oxide ultraviolet absorption spectrum at stratospheric temperatures. Geophys Res Lett 4:427-430.

Silburt, A., Gaidos, E., and Wu, Y. (2015) A statistical reconstruction of the planet population around Kepler solar-type stars. Astrophys $J$ 799, doi:10.1088/0004-637X/799/2/180.

Snellen, I.A.G., de Kok, R.J., de Mooij, E.J., and Albrecht, S. (2010) The orbital motion, absolute mass and high-altitude winds of exoplanet HD209458b. Nature 465:1049-1051.

Snellen, I.A.G., de Kok, R.J., Le Poole, R., Brogi, M., and Birkby, J. (2013) Finding extraterrestrial life using ground- 
based high-dispersion spectroscopy. Astrophys $J 764$ doi: 10.1088/0004-637X/764/2/182.

Snellen, I.A.G., Brandi, B.R., de Kok, R.J., Brogi, M., Birkby, J., and Schwarx, H. (2014) Fast spin of the young extrasolar planet $\beta$ Pictoris b. Nature 509:63-65.

Snellen, I., de Kok, R., Birkby, J.L., Brandl, B., Brogi, M., Keller, C., Kenworthy, M., Schwarz, H., and Stuik, R. (2015) Combining high-dispersion spectroscopy with high contrast imaging: probing rocky planets around our nearest neighbors. Astron Astrophys 576:A59.

Snellen, I., Désert, J.-M., Waters, L.B.F.M., Robinson, T., Meadows, V., van Dishoeck, E.F., Brandi, B.R., Henning, T., Bouwman, J., Lahuis, F., Min, M., Lovis, C., Dominik, C., Van Eylen, V., Sing, D., Anglada-Escudé, G., Birgkby, J.L., and Brogi, M. (2017) Detecting Proxima b's atmosphere with JWST targeting $\mathrm{CO}_{2}$ at $15 \mu \mathrm{m}$ using a highpass spectral filtering technique. Astron J 154, doi:10.3847/ 1538-3881/aa7fbc.

Sparks, W.B. and Ford, H.C. (2002) Imaging spectroscopy for extrasolar planet detection. Astrophys J 578:543-564.

Spiegel, D.S., Raymond, S.N., Dressing, C.D., Scharf, C.A., and Mitchell, J.L. (2010) Generalized Milankovitch cycles and long-term climatic habitability. Astrophys J 721:1308-1318.

Stam, D.M. (2008) Spectropolarimetric signatures of Earth-like extrasolar planets. Astron Astrophys Suppl Ser 482:989-1007.

Stam, D.M., Hovenier, J.W., and Waters, L.B.F. (2004) Using polarimetry to detect and characterize Jupiter-like extrasolar planets. Astron Astrophys Suppl Ser 428:663-672.

Stamenković, V. and Seager, S. (2016) Emerging possibilities and insuperable limitations of exogeophysics: the example of plate tectonics. Astrophys $J$ 825, doi:10.3847/0004-637X/ $825 / 1 / 78$

Stelzer, B., Marino, A., Micela, G., López-Santiago, J., and Liefke, C. (2013) The UV and X-ray activity of the M dwarfs within 10 pc of the Sun. Mon Not R Astron Soc 431:2063-2079.

Sterzik, M.F., Bagnulo, S., and Palle, E. (2012) Biosignatures as revealed by spectropolarimetry of Earthshine. Nature 483: 64-66.

Stevenson, K.B., Désert, J.M., Line, M.R., Bean, J.L., Fortney, J.J., Showman, A.P., Kataria, T., Kreidberg, L., McCullough, P.R., Henry, G.W., and Charbonneau, D. (2014) Thermal structure of an exoplanet atmosphere from phase-resolved emission spectroscopy. Science 346:838-841.

Stevenson, K.B., Lewis, N.K., Bean, J.L., Beichman, C., Fraine, J., Kilpatrick, B.M., Krick, J.E., Lothringier, J.D., Mandell, A.V., Valenti, J.A., Aglo, E., Angerhausen, D., Barstow, J.K., Birkmann, S.M., Burrows, A., Bharbonneau, D., Covan, N.B., Crouzet, N., Cubillos, P.E., Curry, S.M., Dalva, P.A., de Wit, J., Deming, D., Désert, J.-M., Doyon, R., Dragomir, D., Ehrenreich, D., Fortney, J.J., García Muñoz, A., Gibson, N.P., Gizis, J.E., Greene, T.P., Harrington, J., Heng, K., Kataria, T., Kempton, E.G.-R., Knutson, H., Kreidberg, L., Lafrenière, D., Lagage, P.-O., Line, M.R., Lopez-Morales, M., Madhusudhan, N., Morley, C.V., Rocchetto, M., Schlawin, E., Shkolnik, E.L., Shporer, A., Sing, D.K., Todorov, K.O., Tucker, G.S., and Wakeford, H.R. (2016) Transiting exoplanet studies and community targets for JWST's Early Release Science Program. Publ Astron Soc Pac 128, doi: 10.1088/1538-3873/128/967/094401.

Sullivan, P.W., Winn, J.N., Berta-Thompson, Z.K., Charbonneau, D., Deming, D., Dressing, C.D., Latham, D.W., Levine, A.M., McCullough, P.R., Morton, T., Ricker, G.R., Vanderspek, R., and Woods, D. (2015) The Transiting Exoplanet Survey Satellite: simulations of planet detections and astro- physical false positives. Astrophys $J$ 809, doi:10.1088/0004637X/809/1/77.

Swain, M.R. (2012) The FINESSE mission [id. 505.05]. In American Astronomical Society Meeting Abstracts \#220. American Astronomical Society, Washington, DC.

Szentgyorgyi, A., Frebel, A., Furesz, G., Hertz, E., Norton, T., Bean, J., Bergner, H., Crane, J., Evans, J., Evans, I., Gauron, T., Jordán, A., Park, S., Uomoto, A., Barnes, S., Davis, W., Eisenhower, M., Epps, H., Guzman, D. McCracken, K., Ordway, M., Plummer, D., Podgorski, W., and Weaver, D. (2012) The GMT-CfA, Carnegie, Catolica, Chicago Large Earth Finder (G-CLEF): a general purpose optical echelle spectrograph for the GMT with precision radial velocity capability. In: Ground-based and Airborne Instrumentation for Astronomy IV. Proc SPIE 8446, article id. $84461 \mathrm{H}, 15 \mathrm{pp}$.

Tabataba-Vakili, F., Grenfell, J.L., Grießmeier, J.-M., and Rauer, H. (2016) Atmospheric effects of stellar cosmic rays on Earth-like exoplanets orbiting M-dwarfs. Astron Astrophys 585:A96.

Takahashi, J., Itoh, Y., Akitaya, H., Okazaki, A., Kawabata, K., Oasa, Y., and Isogai, M. (2013) Phase variation of Earthshine polarization spectra. Publ Astron Soc Jpn Nihon Tenmon Gakkai 65, doi:10.1093/pasj/65.2.38.

Tamura, M., Suto, H., Nishikawa, J., Kotani, T., Sato, B., Aoki, W., Usuda, T., Kurokawa, T., Kashiwagi, K., Nishiyama, S., Ikeda, Y., Hall, D., Hodapp, K., Hashimoto, J., Morino, J., Inoue, S., Mizuno, Y., Washizaki, Y., Tanaka, Y., Suzuki, S., Kwon, J., Suenaga, T., Oh, D., Narita, N., Kokubo, E., Hayano, Y., Izumiura, H., Kambe, E., Kudo, T., Kusakabe, N., Ikoma, M., Hori, Ya., Omiya, M., Genda, H., Fukui, A., Fujii, Y., Guyon, O., Harakawa, H., Hayashi, M., Hidai, M., Hirano, T., Kuzuhara, M., Machida, M., Matsuo, T., Nagata, T., Ohnuki, H., Ogihara, M., Oshino, S., Suzuki, R., Takami, H., Takato, N., Takahashi, Y., Tachinami, C., and Terada, H. (2012) Infrared Doppler instrument for the Subaru Telescope (IRD). Proc SPIE 8446 , doi: $10.1117 / 12.925885$

Tian, F., France, K., Linsky, J.L., Mauas, P.J.D., and Vieytes, M.C. (2014) High stellar FUV/NUV ratio and oxygen contents in the atmospheres of potentially habitable planets. Earth Planet Sci Lett 385:22-27.

Tinetti, G., Meadows, V.S., Crisp, D., Kiang, N.Y., Kahn, B.H., Bosc, E., Fishbein, E., Velusamy, T., and Turnbull, M. (2006) Detectability of planetary characteristics in disk-averaged spectra II: synthetic spectra and light-curves of Earth. Astrobiology 6:881-900.

Tinetti, G., Drossart, P., Eccleston, P., Hartogh, P., Heske, A., Leconte, J., Micela, G., Ollivier, M., Pilbratt, G., Puig, L., Turrini, D., Vandenbussche, B., Wolkenberg, P., Pascale, E., Beaulieu, J.-P., Güdel, M., Min, M., Rataj, M., Ray, T., Ribas, I., Barstow, J., Bowles, N., Coustenis, A., Coudé du Foresto, V., Decin, L., Encrenaz, T., Forget, F., Friswell, M., Griffin, M., Lagage, P.O., Malaguti, P., Moneti, A., Morales, J.C., Pace, E., Rocchetto, M., Sarkar, S., Selsis, F., Taylor, W., Tennyson, J., Venot, O., Waldmann, I.P., Wright, G., Zingales, T., and Zapatero-Osorio, M.R. (2016) The science of ARIEL (Atmospheric Remote-sensing Infrared Exoplanet Large-survey). Proc SPIE 9904, doi:10.1117/ 12.2232370 .

Traub, W.A. (2012) Terrestrial, habitable-zone exoplanet frequency from Kepler. Astrophys $J$ Volume 745, doi:10.1088/ 0004-637X/745/1/20. 
Traub, W.A. and Oppenheimer, B.R. (2010) Direct imaging of exoplanets. In: Exoplanets, edited by S. Seager. Tucson, AZ: University of Arizona Press, pp 111-156.

Trauger, J., Moody, D., and Gordon, B. (2013) Complex apodized Lyot coronagraph for exoplanet imaging with partially obscured telescope apertures. Proc SPIE 8864, doi:10.1117/ 12.2024795 .

Turbet, M., Leconte, J., Selsis, F., Bolmont, E., Forget, F., Ribas, I., Raymond, S.N., and Anglada-Escudé, G. (2016) Astron Astrophys 596, id. A112, 29 pp.

Vandaele, A.C., Hermans, C., Simon, P.C., Carleer, M., Colin, R., Fally, S., Mérienne, M.F., Jenouvrier, A., and Coquart, B. (1998) Measurements of the $\mathrm{NO}_{2}$ absorption crosssection from $42,000 \mathrm{~cm}^{-1}$ to $10,000 \mathrm{~cm}^{-1}(238-1000 \mathrm{~nm})$ at 220 K and 294 K. J Quant Spectrosc Radiat Transf 59: 171-184.

Vidal-Madjar, A., Lecavelier des Etangs, A., Désert, J.-M., Ballester, G.E., Ferlet, R., Hébrard, G., and Mayor, M. (2003) An extended upper atmosphere around the extrasolar planet HD209458b. Nature 422:143-146.

Vigan, A., Gry, C., Salter, G., Mesa, D., Homeier, D., Moutou, C., and Allard, F. (2015) High-contrast imaging of Sirius A with VLT/SPHERE: looking for giant planets down to one astronomical unit. Monthly Notices of the Royal Astronomical Society 454:129-143.

von Paris, P., Hedelt, P., Selsis, F., Schreier, F., and Trautmann, T. (2013) Characterization of potentially habitable planets: retrieval of atmospheric and planetary properties from emission spectra. Astron Astrophys 551: A120.

Walker, S.I., Bains, W., Cronin, L., DasSarma, S., Danielache, S., Domagal-Goldman, S., Kacar, B., Kiang, N.Y., Lenardic, A., Reinhard, C.T., Moore, W., Schwieterman, E.W., Shkolnik, E.L., and Smith, H.B. (2018) Exoplanet biosignatures: future directions. Astrobiology 18:779-824.

Wang, J., Mawet, D., Ruane, G., Hu, R., and Benneke, B. (2017) Observing exoplanets with high dispersion coronagraphy. I. The scientific potential of current and nextgeneration large ground and space telescopes. Astron J 153, doi:10.3847/1538-3881/aa6474.

Weiss, L.M. and Marcy, G.W. (2014) The mass-radius relation for 65 exoplanets smaller than 4 Earth radii. Astrophys $J$ 783:L6.

West, A.A., Hawley, S.L., Bochanski, J.J., Covey, K.R., Reid, I.N., Dhital, S., Hilton, E.J., and Masuda, M. (2008) Constraining the age-activity relation for cool stars: the Sloan Digital Sky Survey Data Release 5 low-mass star spectroscopic sample. Astron J 135:785-795.

Wheatley, P.J., Pollacco, D.L., Queloz, D., Rauer, H., Watson, C.A., West, R.G., Chazelas, B., Louden, T.M., Walker, S., Bannister, N., Bento, J., Burleigh, M., Cabrera, J., Eigmüller, P., Erikson, A., Genolet, L., Goad, M., Grange, A., Jordán, A., Lawrie, K., McCormac, J., and Neveu, M. (2013) The Next Generation Transit Survey (NGTS). In Hot Planets and Cool Stars, edited by R. Saglia, EPJ Web of Conferences, Vol. 47, doi:10.1051/epjconf/20134713002.

Wiktorowicz, S.J. (2009) Nondetection of polarized, scattered light from the HD 189733b hot Jupiter. Astrophys J 696: 1116-1124.

Wiktorowicz, S.J., Nofi, L.A., Jontof-Hutter, D., Kopparla, P., Laughlin, G.P., Hermis, N., Yung, Y.L., and Swain, M.R. (2015) A ground-based albedo upper limit for HD 189733 b from polarimetry. Astrophys $J$ 813, Issue 1, article id. $48,11 \mathrm{pp}$.
Williams, D.M. and Gaidos, E. (2008) Detecting the glint of starlight on the oceans of distant planets. Icarus 195:927-937. Wilson, O.C. (1966) Stellar chromospheres. Science 151:14871498.

Wolf, E.T. (2017) Assessing the habitability of the TRAPPIST1 system using a 3D climate model. Astrophys $J$ 839:L1.

Wolf, E.T. and Toon, O.B. (2014) Delayed onset of runaway and moist greenhouse climates for Earth. Geophys Res Lett 41:167-172.

Wolf, E.T. and Toon, O.B. (2015) The evolution of habitable climates under the brightening Sun. J Geophys Res: Atmospheres 120:5775-5794.

Wordsworth, R.D., Forget, F., Selsis, F., Millour, E., Charnay, B., and Madeleine, J.-B. (2011) Gliese 581d is the first discovered terrestrial-mass exoplanet in the habitable zone. Astrophys $J$ 733:L48.

Wright, J.T. (2005) Radial velocity jitter in stars from the California and Carnegie planet search at Keck Observatory. Publ Astron Soc Pac 117:657-664.

Wright, J.T., Marcy, G.W., Butler, R.P., and Vogt, S.S. (2004) Chromospheric Ca II emission in nearby F, G, K, and M stars. Astrophys J Suppl Ser 152, doi:10.1086/386283.

Wright, N.J., Drake, J.J., Mamajek, E.E., and Henry, G.W. (2011) The stellar-activity-rotation relationship and the evolution of stellar dynamos. Astrophys $J$ 743:48.

Yang, J., Cowan, N.B., and Abbot, D.S. (2013) Stabilizing cloud feedback dramatically expands the habitable zone of tidally locked planets. Astrophys J 771:L45.

Yoshino, K., Cheung, A.S.C., Esmond, J.R., Parkinson, W.H., Freeman, D.E., Guberman, S.L., Jenouvrier, A., Coquart, B., and Merienne, M.F. (1988) Improved absorption crosssections of oxygen in the wavelength region $205-240 \mathrm{~nm}$ of the Herzberg continuum. Planetary and Space Science 36: 1469-1475.

Youngblood, A., France, K., Parke Loyd, R.O., Linsky, J.L., Redfield, S., Schneider, P.C., Wood, B.E., Brown, A., Froning, C., Miguel, Y., Rugheimer, S., and Walkowicz, L. (2016) The MUSCLES Treasury Survey. II. Intrinsic Ly $\alpha$ and extreme ultraviolet spectra of $\mathrm{K}$ and $\mathrm{M}$ dwarfs with exoplanets. Astrophys $J$ 824, doi:10.3847/0004-637X/824/2/101.

Zsom, A. (2015) A population-based habitable zone perspective. Astrophys J 813, doi:10.1088/0004-637X/813/1/9.

Zsom, A., Seager, S., De Wit, J., and Stamenković, V. (2013) Toward the minimum inner edge distance of the habitable zone. Astrophys J 778, doi:10.1088/0004-637X/778/2/109.

Zugger, M.E., Kasting, J.F., Williams, D.M., Kane, T.J., and Philbrick, C.R. (2010) Light scattering from exoplanet oceans and atmospheres. Astrophys $J$ 723:1168-1179.

Zugger, M.E., Kasting, J.F., Williams, D.M., Kane, T.J., and Philbrick, C.R. (2011) Searching for water earths in the near-infrared. Astrophys J 739, doi:10.1088/0004-637X/ $739 / 1 / 12$

Address correspondence to: Yuka Fujii

Earth-Life Science Institute Tokyo Institute of Technology 2-12-1 I7E-307 Ookayama Meguro, Tokyo 152-8550 Japan

E-mail: yuka.fujii@elsi.jp

Submitted 3 August 2017

Accepted 13 March 2018 


$\begin{aligned} & \text { Abbreviations Used } \\ & \text { ARIEL }= \text { Atmospheric Remote-sensing Infrared } \\ & \text { Exoplanet Large-survey } \\ & \text { CGI }=\text { WFIRST coronagraph instrument } \text { CHaracterising ExOPlanet Satellite } \\ & \text { CHEOPS }=\text { CHaph } \\ & \text { CRIRES }=\text { Cryogenic Infrared Echelle Spectrograph } \\ & \text { ELT }=\text { European-Extremely Large Telescope } \\ & \text { ELTs }=\text { Extremely Large Telescopes } \\ & \text { ESPRESSO }=\text { Echelle SPectrograph for Rocky Exoplanet } \\ & \quad \text { and Stable Spectroscopic Observations } \\ & \text { FINESSE }=\text { Fast Infrared Exoplanet Spectroscopy } \\ & \quad \text { Survey Explorer } \\ & \text { GMT }=\text { Giant Magellan Telescope } \\ & \text { HabEx }=\text { Habitable Exoplanet Imaging Mission } \\ & \text { HARPS }=\text { High Accuracy Radial velocity Planet } \\ & \text { Searcher } \\ & \text { HST }=\text { Hubble Space Telescope } \\ & \text { HZs }=\text { habitable zones } \\ & \text { IWA }=\text { inner working angle } \\ & \text { JWST }=\text { James Webb Space Telescope } \\ & \text { K2 }=\text { the repurposed Kepler spacecraft }\end{aligned}$

